\title{
ESTIMACIÓN DE REACTORES DE HOMOPOLIMERIZACIÓN EN SOLUCIÓN: \\ EFECTO DE LA ESTRUCTURA \\ DE ESTIMABILIDAD
}

\author{
TESIS QUE PARA OBTENER EL GRADO DE \\ MAESTRO EN CIENCIAS (INGENIERÍA QUIIMICA) \\ PRESENTA
}

PABLO GONZÁLEZ CERVANTES

SEPTIEMBRE DE 2000

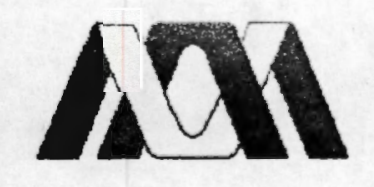

UNIVERSIDAD AUTÓNOMA METROPOLITANA UNIDAD IZTAPALAPA

DIVISIÓN DE CIENCIAS BÁSICAS E INGENIERÍA 
Asesor:

Dr. Jesús Alvarez Calderón

Sinodales:

Presidente: Dr. Roberto Olayo González

(UAM - I)

Secretario:

Dr. Jesús Alvarez Calderón

(UAM - I)

Vocal:

M.I.Q. Fernando Zaldo García

(CIP)

Este trabajo de investigación se desarrolló mediante el apoyo de una Beca - Crédito del Consejo Nacional de Ciencia y Tecnología (CONACyT), y de una Beca - Galardón del Centro de Investigación en Polímeros (CIP). 


\section{AGRADECIMIENTOS}

A mi asesor:

\section{Dr. Jesús Alvarez Calderón}

Por su invaluable aportación durante el tiempo en que desarrollé este trabajo de investigación. Gracias por cada asesoría en la que he aprendido algo valioso.

\section{A mi madre:}

\section{Minerva Cervantes Del Ángel}

Porque gran parte de lo que soy a ella se lo debo. Porque siempre tendré en mente su amor y apoyo incondicional y todas las ocasiones en que vino a visitarme durante mis estudios de maestría. Gracias de todo corazón.

\section{A mi padre:}

\section{Pablo González Santamán}

Por su influencia sobre mi persona, su apoyo durante mis estudios previos y el amor que me ha brindado. Gracias por haberme fomentado el gusto por las matemáticas.

\section{A mi mejor amigo:}

\section{Itzcoatl Alfonso Plá Hemández}

Por su amistad y las horas de estudio compartidas en los primeros trimestres de la maestría. Gracias amigo.

A mi hermana y mejor amiga:

\section{Argelia González Cervantes}

Por sus palabras de aliento, amor y humor, recibidas a través de sus cartas. Gracias Arge.

\section{A mi prometida:}

\section{Sandra Pérez Correa}

Por su motivación, amor e infinitos momentos de felicidad. Todo ello me dio el impulso de seguir adelante. Te amo. 


\section{INDICE}

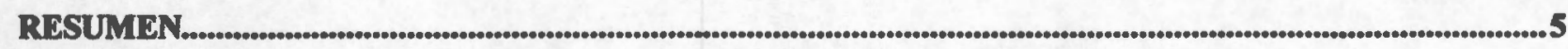

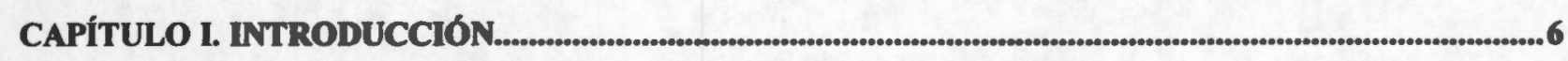

CAPÍTULO II. REACTOR DE POLIMERIZACIÓN Y SU PROBLEMA DE ESTIMACIÓN.........................9

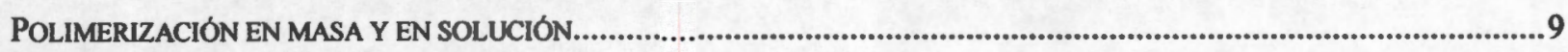

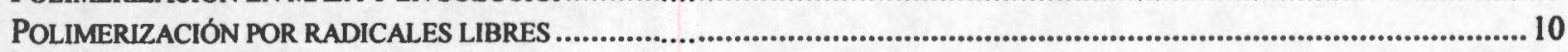

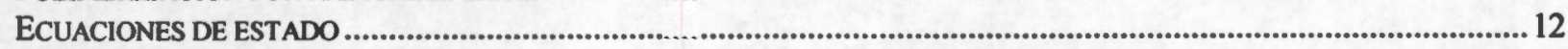

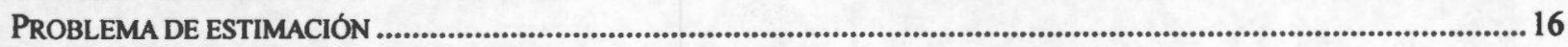

CAPÍTULO III. ESTIMACIÓN NO LINEAL_.................................................................................................. 17

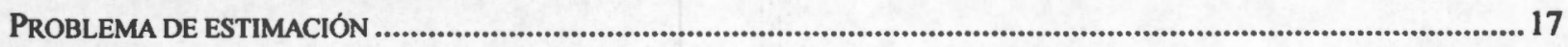

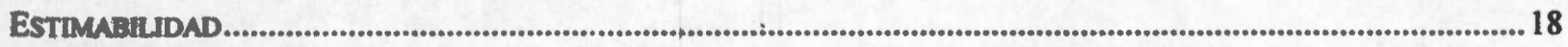

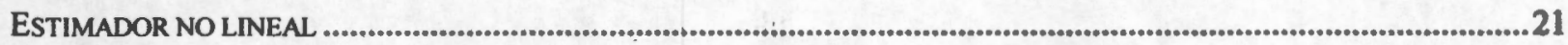

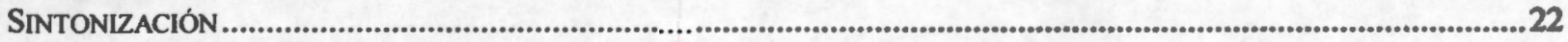

CAPITULO IV. RESOLUBILIDAD DEL PROBLEMA DE ESTIMACIÓN ............................................. 24

MEDICIONES: CONCENTRACIÓN DE MONÓMERO, TEMPERATURA Y VOLUMEN ............................................................24

MEDICIONES: DENSIDAD/ÍNDICE DE REFRACCIÓN, TEMPERATURA Y VOLUMEN . ........................................................31

MEDICIONES: ÍNDICE DE REFRACCIÓN, DENSIDAD, TEMPERATURA Y VOLUMEN ....................................................36

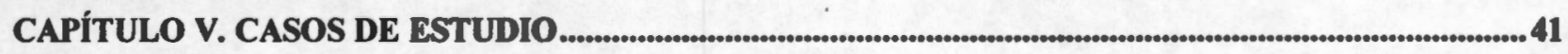

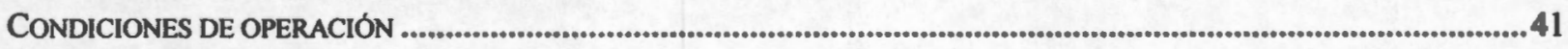

MAPAS DE SALIDA.

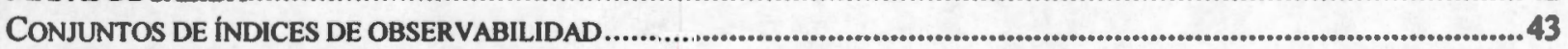

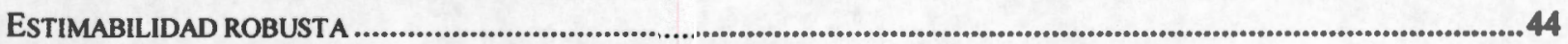

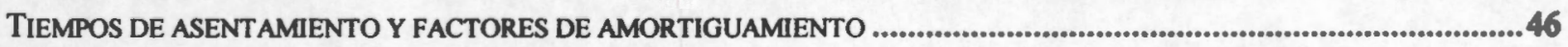

REACTOR EN LOTE

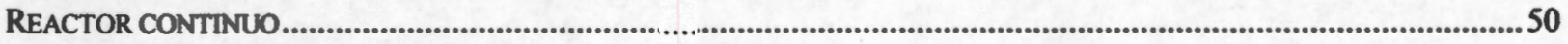

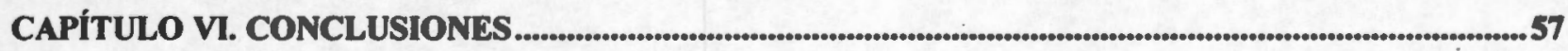

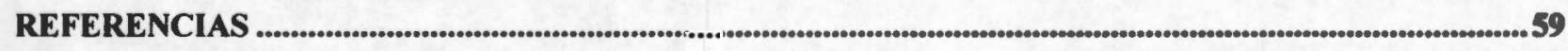

APÉNDICE A. VECTORES Y MATRICES DEL ESTIMADOR NO LINEAL ...........................................6 61

APÉNDICE B. MODELO DE HOMOPOLIMERIZACIÓN EN SOLUCIÓN POR RADICALES LIBRES.62

APÉNDICE C. INDICE DE REFRACCIÓN.

APÉNDICE D. DEDUCCIÓN DE LAS VARIABLES DE INTERÉS PARA UN REACTOR DE HOMOPOLIMERIZACIÓN EN SOLUCIÓN.............................................................................................................80

APÉNDICE E. CONSTRUCCIÓN Y SINTONIZACIÓN DE LOS ESTIMADORES NO LINEALES ...........86 


\section{RESUMEN}

En este proyecto de investigación de tesis de maestría se estudió el problema de estimación para reactores de homopolimerización en solución vía radicales libres, con énfasis en la propiedad de estimabilidad analizada en función de las mediciones y la estructura de estimabilidad (conjuntos de índices de observabilidad y estados de la dinámica observable); Se abordó el caso general de polimerización en solución, considerando a la polimerización en masa es un caso particular.

El estudio del problema de estimación abarcó el análisis de la resolubilidad del problema, la construcción y sintonización de los estimadores, y verificación del desempeño de los estimadores.

La resolubilidad se analizó en función de las mediciones (índice de refracción y/o densidad, temperatura y volumen) y los conjuntos posibles de índices de observabilidad. Se concluyó que en el proceso estudiado, las trayectorias del reactor son robusta y exponencialmente estimables para determinados conjuntos de índices de observabilidad. Asimismo se determinó que analíticamente los estados de la dinámica observable son la concentración de monómero, la temperatura, el volumen y la concentración de iniciador, con base en consideraciones físicas e ingenieriles. La construcción y sintonización de los estimadores se realizó de acuerdo a procedimientos sistemáticos; se construyeron dos estimadores en base a diferentes índices de observabilidad para la densidad ( 1 y 2 ). La verificación del desempeño se realizó para un reactor en lote (con mediciones experimentales de densidad y volumen), y un reactor continuo (con mediciones experimentales de densidad y temperatura) y se compararon las estimaciones con datos reportados (conversión y pesos moleculares en número y en peso).

El estimador construido con índice de observabilidad igual a 1 presenta un mejor desempeño en comparación con el construido con índice igual a 2, manifestándose tal mejoría en la estimación de propiedades ligadas a la dinámica no observable (pesos moleculares); ambos estimadores manifiestan comportamiento similar en la predicción de propiedades ligadas con la dinámica observable (conversión, fracción masa de sólidos). El aumento de índice 1 a 2 implica que el iniciador se incluye en la dinámica observable; sin embargo, la inclusión de iniciador en la misma no conduce a un mejor desempeño. El desempeño se degrada por la propagación de errores de modelado (ya que inexorablemente se tiene un modelo aproximado). 


\section{Capítulo I. Introducción}

El estudio de reactores de polimerización resulta de interés ya que mediante estos equipos de proceso se obtienen productos de aplicación comercial (poliestireno, cloruro de polivinilo, polimetilmetacrilato) a la vez que se pueden presentar problemas de eliminación de calor y aumento de viscosidad alcanzando condiciones riesgosas de operación; en estos reactores se busca garantizar una velocidad óptima de producción, producto dentro de especificaciones y seguridad en la operación. La conversión y el peso molecular son indicadores de la velocidad de producción y calidad de producto, en tanto que la temperatura, volumen y fracción masa de sólidos muestran la existencia de condiciones riesgosas.

El conocimiento de tales propiedades requiere del conocimiento de los estados del sistema (concentraciones, temperatura, volumen...), mas en una situación práctica no es posible contar con mediciones de todos los estados, por lo que se requiere de procesadores dinámicos de datos (estimadores no lineales) los que a partir del conocimiento de las entradas exógenas y las mediciones disponibles, infieren en línea los estados y variables de interés (conversión, fracción de sólidos, pesos moleculares).

La estimación no lineal de estados es un tema ampliamente estudiado en ingeniería de procesos, con implicaciones en control convencional o avanzado, control supervisorio, monitoreo de calidad de producto, detección de fallas, reconciliación de datos, rediseño de procesos y demás. Los principales enfoques para el diseño de estimadores no lineales son los siguientes (Alvarez y López, 1999): (i) el filtro extendido de Kalman, FEK (Jazwinski, 1970), cuyo diseño es simple pero carece de criterios de convergencia y procedimientos sistemáticos de sintonización; (ii) el diseño de observador geométrico, OG (Krener e Isidori, 1983), el cual garantiza la convergencia con dinámicas lineales de los errores de salida pero es aplicable a una clase de plantas extremadamente restringida; (iii) el diseño de alta ganancia, AG (Gauthier y col. 1992; Ciccarella y col. 1993), que garantiza convergencia pero tiene procedimientos complejos de sintonización; y (iv) el diseño de modo deslizante, MD (Slotine y col. 1987) que garantiza estabilidad robusta pero tiene un procedimiento muy elaborado. Las técnicas FEK, GO, AG y MD están restringidas a plantas observables (Krener y Respondek, 1985). 
De las técnicas anteriores de estimación, el FEK es la más empleada en ingeniería química (Ellis y col., 1988, 1994; Mutha y col., 1997). Alvarez (1997) desarrolló un esquema de estimación que combina las técnicas de un observador geométrico y la aproximación de alta ganancia incluyendo un método sistemático de sintonización mediante localización de polos y criterios robustos de convergencia; el esquema de estimación es aplicable a sistemas observables o detectables. Las mediciones empleadas son de naturaleza continua e instantánea (tales como la densidad, la temperatura y el volumen).

Alvarez y López (1999) diseñaron un esquema de estimación no lineal basado en el trabajo de Alvarez (1997), con la diferencia de que la propiedad de estimabilidad se deriva de una forma constructiva, en vez de ser una generalización de la requerida en el diseño del observador convergente de Ciccarella (1993). En el diseño del estimador se contempla una etapa de análisis, en la que se verifica la estimabilidad de las trayectorias de los estados, y una etapa de síntesis en la que se construye el estimador y se sintonizan las ganancias. Se aplicó el esquema de estimación a un reactor de homopolimerización en masa (al nivel de simulación), variando la dimensión del mapa de observabilidad. Se determinó que el conjunto de índices de observabilidad afecta la robustez del estimador.

En resumen, aunque se han aplicado diversos esquemas de estimación no lineal en ingeniería química, y particularmente para reactores de polimerización, es necesario resaltar que la construcción de un estimador no garantiza la convergencia de los estados estimados hacia los de la planta. Es necesario realizar un estudio previo sobre la resolubilidad del problema de estimación cuando se cuenta con diferentes estructuras de estimabilidad y mediciones. Alvarez y López (1999) realizaron el estudio para una homopolimerización en masa; sin embargo, es necesario el estudio del caso general de homopolimerización en solución, en el entendido de que el proceso en masa es un caso particular en el que la concentración de solvente es nula, y por tanto, las conclusiones obtenidas para el caso en solución contendrá las conclusiones particulares para el caso en masa. Asimismo, como un paso adelante al trabajo al nivel de simulación, se requiere que el desempeño de estimadores no lineales se apoye con validación experimental.

En este trabajo se estudió el problema de estimación para reactores de homopolimerización en solución vía radicales libres, con énfasis en la propiedad de estimabilidad analizada en función de las mediciones y la estructura de estimabilidad; la validación experimental se realizó mediante el 
empleo de datos provenientes de literatura. Se abordó el caso general de polimerización en solución, considerando que la polimerización en masa es un caso particular y que las conclusiones obtenidas para el caso en solución contienen las conclusiones particulares para el caso en masa.

La metodología seguida fue la siguiente:

(1) A partir de un procedimiento estándar de balances de materia y energía, se estableció un modelo para la descripción del proceso. El sistema considerado fue un reactor continuo de tanque agitado, donde se verifica una reacción de homopolimerización en solución cuando el mecanismo cinético es el de radicales libres. Asimismo, se delimitó el problema de estimación para esta clase de procesos, como un problema de inferencia de variables que describen la velocidad de producción, calidad de producto y seguridad en la operación del reactor.

(2) Con base en el trabajo de Alvarez y López (1999), se analizó la resolubilidad del problema de estimación en función de la estructura de estimabilidad y las mediciones, y se realizó la construcción y sintonización de los estimadores. Las mediciones son de naturaleza contínua e instantánea (densidad, índice de refracción, temperatura y volumen); la construcción y sintonización de los estimadores son procedimientos sistemáticos y con base en la dinámica del proceso.

(3) Se estudió el desempeño de los estimadores utilizando mediciones experimentales, mapas de salida y datos cinéticos provenientes de la literatura. Se analizó el desempeño comparando las estimaciones contra los datos de dos sistemas experimentales reportados en la literatura. Se emplearon mediciones experimentales (densidad, temperatura, volumen) para la estimación de conversión y pesos moleculares. 


\section{Capítulo II. Reactor de polimerización y su problema de estimación}

En este capítulo se presentará la descripción de un tipo de reacción de polimerización (vía radicales libres) estándar en literatura, para después abordar las ecuaciones diferenciales que describen los estados de un reactor de polimerización, haciendo mención a las entradas exógenas y las mediciones disponibles. Se concluirá con la definición del problema de estimación en este tipo de reactores, para la inferencia de variables indicadoras de seguridad de operación, velocidad de producción y calidad de producto.

\section{Polimerización en masa y en solución}

La producción de un polímero puede realizarse en un medio homogéneo o heterogéneo. Cuando se considera un medio homogéneo, el proceso puede ser en solución o en masa; los medios heterogéneos se refieren a si la polimerización se realiza en suspensión o en emulsión. El medio en que se realiza la polimerización varía de acuerdo a la naturaleza del monómero y la utilización que se vaya a hacer del polímero. Por el momento se limitará al análisis de las polimerizaciones en medios homogéneos.

La polimerización en masa de un monómero puro es el proceso más simple en el que se minimizan los problemas de contaminación del producto. El método se emplea para obtener directamente el polímero con la forma de un molde (Uribe, 1990). A pesar de evitar los problemas de contaminación, el proceso es difícil de controlar debido a las características de la polimerización. Su naturaleza exotérmica, las altas energías de activación involucradas, y la tendencia hacia el efecto gel provocan una dificultad en la eliminación del calor. El aumento en viscosidad con la conversión es otro problema involucrado, por lo que se debe emplear un sistema eficiente de agitación y eliminación del calor. El incremento en viscosidad puede provocar la aparición de "zonas calientes" (hot spots) con el consiguiente aumento en la polidispersidad del producto.

La polimerización en solución evita los problemas de la polimerización en masa relacionados con la viscosidad y eliminación de calor, lo que se debe a la presencia de solvente que actúa como diluyente, facilita la agitación y ayuda en la transferencia de calor. En la polimerización en solución 
es más fácil el control térmico; sin embargo, puede requerir (a) gran cantidad de solvente para limitar la viscosidad del medio, y (b) operaciones de separación del polímero y recuperación. Por otra parte, diversas reacciones con el solvente pueden conducir a la reducción en la calidad del polímero (reducción del peso molecular). Este tipo de polimerización se emplea especialmente cuando el polímero se aplicará en forma disuelta, como los adhesivos o barnices.

\section{Polimerización por radicales libres}

Entre las reacciones de polimerización destacan las que se llevan a cabo mediante radicales libres, ya que presentan características comunes a la mayoría de las reacciones de polimerización. Una reacción de polimerización por radicales libres se caracteriza por la gran cantidad de calor generado, el aumento de la viscosidad con la conversión y la disminución de la capacidad para la eliminación de calor; como resultado se verifica una autoaceleración de la reacción. Este conjunto de fenómenos se denominan "efecto gel" (Chiu y col., 1983). La reacción de polimerización puede realizarse en masa o en solución; aunque la polimerización en masa presenta los problemas más severos de aumento de viscosidad y eliminación de calor, este tipo de polimerización puede considerarse un caso particular de la polimerización en solución.

La polimerización por radicales libres es una reacción en cadena que básicamente consta de iniciación, propagación, y terminación.

\section{Iniciación}

La iniciación involucra dos pasos. En primer término un iniciador de la reacción I se disocia en dos radicales $R$ :

$$
\mathrm{I} \stackrel{\mathrm{k}_{1}}{\longrightarrow} 2 \mathrm{R}
$$

en donde $k_{d}$ es la constante de disociación del iniciador. En la segunda etapa cada radical $R$ reacciona con una molécula de monómero $M$ para producir una molécula activa $P_{1}$, la cual será una especie iniciadora de una cadena.

$$
\mathrm{R}+\mathrm{M} \stackrel{\mathbf{k}_{i}}{\longrightarrow} \mathrm{P}_{1}
$$

\section{Propagación}

La propagación consiste en el crecimiento de cadenas a partir de $\boldsymbol{P}_{\boldsymbol{l}}$ mediante la adición repetida de moléculas $M$, de forma que con la adición de cada una se forma un radical o cadena activa que es mayor en una unidad monomérica que la anterior. La propagación se realiza de la siguiente 
forma:

$$
\begin{aligned}
& P_{1}+M \stackrel{k_{p}}{\longrightarrow} P_{2}+\left(-\Delta H_{p}\right) \\
& P_{2}+M \stackrel{k_{p}}{\longrightarrow} P_{3}+\left(-\Delta H_{p}\right) \\
& P_{3}+M \stackrel{k_{p}}{\longrightarrow} P_{4}+\left(-\Delta H_{p}\right)
\end{aligned}
$$

y en general:

$$
\mathrm{P}_{\mathrm{i}}+\mathrm{M} \stackrel{\mathrm{k}_{\mathrm{p}}}{\longrightarrow} \mathrm{P}_{\mathrm{i}+1}+\left(-\Delta \mathrm{H}_{\mathrm{p}}\right)
$$

en donde $k_{p}$ es la constante de propagación. Las propiedades termodinámicas de una polimerización (como $\Delta H$ ) se relacionan principalmente con el paso de propagación, ya que en la polimerización existen numerosos pasos de propagación mientras que la iniciación y terminación son etapas únicas (Odian, 1991).

\section{Terminación}

La cadena polimérica activa $P_{i}$ puede detener su crecimiento en cierto momento, lo que se denomina terminación. La terminación puede ocurrir de dos formas: por combinación o por desproporción; en ambas la reacción ocurre entre dos cadenas activas. La terminación por combinación ocurre por la condensación de dos cadenas activas en una sola inactiva $\left(D_{i+j}\right)$ :

$P_{i}+P_{j} \stackrel{k_{k s}}{\longrightarrow} D_{i+j}$

en tanto que en la terminación por desproporción ocurre la transferencia de un radical hidrógeno (de posición beta) de una cadena a otra, con la consiguiente inactivación del radical de una cadena (molécula polimérica saturada) y la formación de un doble enlace en la otra (molécula polimérica insaturada):

$$
\mathrm{P}_{\mathrm{i}}+\mathrm{P}_{\mathrm{j}} \stackrel{\mathrm{k}_{\mathrm{u}}}{\longrightarrow} \mathrm{D}_{\mathrm{i}}+\mathrm{D}_{\mathrm{j}}
$$

En varios sistemas de polimerización puede ocurrir que el peso molecular predicho no corresponda con el experimental; es decir, la cadena activa termina su crecimiento antes de lo esperado debido a la transferencia de un hidrógeno u otra especie por parte de otro compuesto del sistema, como puede ser el iniciador, el monómero o el solvente. Tales reacciones de desplazamiento de radical se conocen como reacciones de transferencia.

Transferencia de cadena al monómero

$$
\mathrm{P}_{\mathrm{i}}+\mathrm{M} \stackrel{\mathrm{k}_{\mathrm{m}}}{\longrightarrow} \mathrm{D}_{\mathrm{i}}+\mathrm{P}_{\mathrm{i}}
$$


Las constantes de transferencia a monómero $\left(k_{\rho M}\right)$ son generalmente pequeñas para la mayoría de los monómeros (Odian, 1991). Las mayores constantes de transferencia se observan cuando las cadenas activas tienen alta reactividad, por ejemplo, para el etileno, el acetato de vinilo y el cloruro de vinilo.

Transferencia de cadena al solvente

$\mathrm{P}_{\mathrm{i}}+\mathrm{S} \stackrel{\mathrm{k}_{\mathrm{k}}}{\longrightarrow} \mathrm{D}_{\mathrm{i}}+\mathrm{P}_{\mathrm{i}}$

En general, el valor absoluto de la constante de transferencia a solvente $\left(k_{f s}\right)$ depende del monómero que es polimerizado. Este efecto es una consecuencia de la reactividad del radical del monómero. La constante de transferencia a monómero para cualquier compuesto generalmente se incrementa con la reactividad del radical. El orden de reactividad del radical es el siguiente: cloruro de vinilo $>$ acetato de vinilo $>$ acrilonitrilo $>$ metil acrilato $>$ metil metacrilato $>$ estireno $>$ 1,3 - butadieno.

Transferencia de cadena a un agente de transferencia

Este tipo de compuestos provoca reacciones de transferencia y se utilizan para disminuir el peso molecular del polímero. Generalmente son mercaptanos con cadena alifática. La reacción ocurre de la siguiente manera:

$P_{i}+W \stackrel{N_{m}}{\longrightarrow} D_{i}+P_{i}$

donde $W$ es el agente de transferencia.

\section{Ecuaciones de estado}

Considérese un reactor de homopolimerización por radicales libres en solución y su esquema de estimación (fig. 1.1). La reacción se ve afectada por el efecto gel (alta exotermicidad, incremento de viscosidad con la conversión, limitación de la eliminación de calor y autoaceleración de la reacción). 


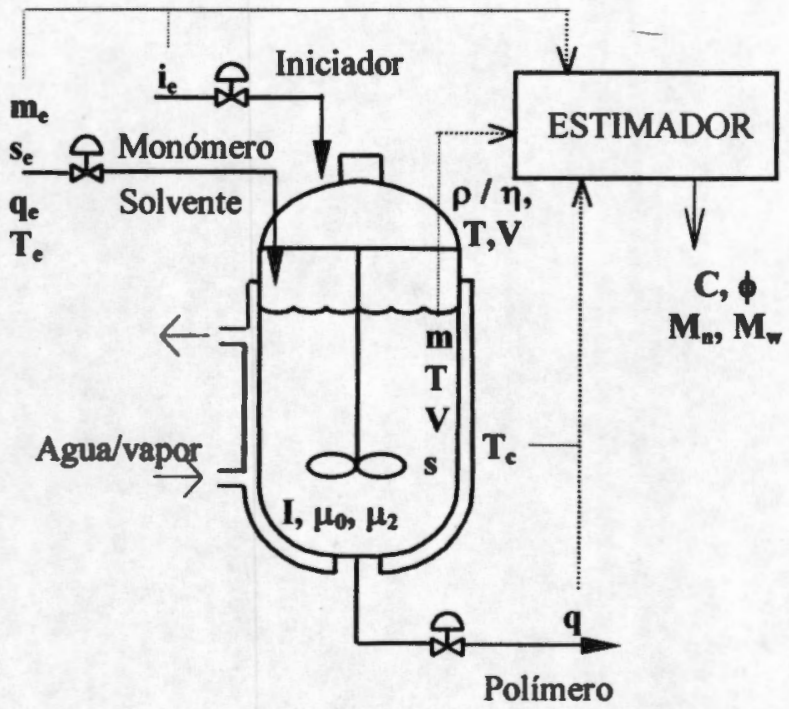

Figura. 1.1 Reactor de homopolimerización y su esquema de estimación

Para la obtención del conjunto de ecuaciones diferenciales que describen los estados del reactor, se realizan 3 suposiciones principales relacionadas con la cinética:

a) Los radicales activos $P_{i}$ del polimero tienen igual reactividad independientemente de su longitud de cadena. Es decir, la constante $k_{p}$ es independiente de la longitud de cadena.

b) Hipótesis de cadena cinética larga. Cada radical participa en un gran número de reacciones de propagación antes de la terminación. Esto implica que el consumo de monómero por propagación hace que el consumo de monómero por iniciación sea despreciable.

c) Hipótesis de estado cuasiestacionario. La velocidad de iniciación (formación de centros activos) es igual a la velocidad de terminación (desaparición de centros activos). Se establece rápidamente un estado estacionario de la concentración de los radicales $\boldsymbol{P}_{i}$, en virtud de su reactividad.

En la deducción del modelo se utilizan otras suposiciones:

1. El reactor de tanque agitado tiene mezclado ideal, es decir, no existen gradientes espaciales de temperatura y concentración.

2. La dinámica de la camisa es muy rápida.

3. Las constantes de reacción tienen una dependencia tipo Arrhenius con respecto de la temperatura. 
4. El calor generado proviene principalmente de la reacción de propagación.

5. La conductividad térmica y la capacidad calorífica del medio permanecen constantes e independientes de la temperatura.

6. Los volúmenes específicos del monómero, solvente y polímero son aditivos.

Mediante el anterior mecanismo cinético (2.1) y basándose en la funcionalidad del efecto gel y la descripción de la viscosidad y coeficiente de transporte de calor (Bondy y Lippa, 1983; Alvarez y col., 1990), la deducción (mediante procedimientos estándares) del modelo del reactor conduce a 7 ecuaciones diferenciales ordinarias no lineales (funcionalidades en el apéndice B):

$$
\begin{aligned}
& I=-r_{l}(I, T)+\left\{\varepsilon_{m} r_{p o l}(I, m, s, T)+\varepsilon_{s} r_{s o l}(I, m, s, T)\right\}+\frac{\left(i_{e}-q_{e} I\right)}{V}:=f_{i} \\
& s=-r_{s o l}(I, m, s, T)+\left\{\varepsilon_{m} r_{p o l}(I, m, s, T)+\varepsilon_{s} r_{s o l}(I, m, s, T)\right\}+\frac{q_{e}}{V}\left(s_{e}-s\right):=f_{s} \\
& \mathrm{~m}=-\mathrm{r}_{\mathrm{pol}}(\mathrm{I}, \mathrm{m}, \mathrm{s}, \mathrm{T})+\left\{\varepsilon_{\mathrm{m}} \mathrm{r}_{\mathrm{pol}}(\mathrm{I}, \mathrm{m}, \mathrm{s}, \mathrm{T})+\varepsilon_{\mathrm{s}} \mathrm{r}_{\mathrm{sol}}(\mathrm{I}, \mathrm{m}, \mathrm{s}, \mathrm{T})\right\}_{\mathrm{m}}+\frac{\mathrm{q}_{\mathrm{e}}}{V}\left(\mathrm{~m}_{\mathrm{e}}-\mathrm{m}\right):=\mathrm{f}_{\mathrm{m}} \\
& T=\beta(m, s) r_{p}(I, m, s, T)-\gamma(m, s, T, V)\left(T-T_{c}\right)+\frac{q_{e}}{V}\left(\frac{1-\varepsilon_{m} m_{e}-\varepsilon_{s} s_{e}}{1-\varepsilon_{m} m-\varepsilon_{s} s}\right)\left(T_{e}-T\right):=f_{T} \\
& \left.\nabla=-\varepsilon_{m} r_{p o l}(I, m, s, T)+\varepsilon_{s} r_{s o l}(I, m, s, T)\right\} V+q_{c}-q:=f_{V} \\
& \mu_{0}=r_{\mu 0}(I, m, s, T)+\left\{\varepsilon_{m} r_{p o l}(I, m, s, T)+\varepsilon_{s} r_{s o l}(I, m, s, T)\right\}_{\mu_{0}}+\frac{q_{e}}{V}\left(\mu_{0 e}-\mu_{0}\right):=f_{\mu 0} \\
& \mu_{2}=r_{\mu 2}(I, m, s, T)+\left\{\varepsilon_{\mathrm{m}} r_{p o l}(I, m, s, T)+\varepsilon_{s} r_{s o l}(I, m, s, T) \mu_{2}+\frac{q_{e}}{V}\left(\mu_{2 e}-\mu_{2}\right):=f_{\mu 2}\right. \\
& y_{1}=\rho / \eta \\
& \mathrm{y}_{2}=\mathrm{T} \\
& \mathrm{y}_{3}=\mathrm{V} \\
& u=\left[i_{e}, q_{e}, q, T_{e}, T_{c}, m_{e}, s_{e}, \mu_{0_{e}}, \mu_{2 e}\right]^{T}
\end{aligned}
$$

En notación compacta se puede representar el modelo del reactor de la siguiente forma: $\dot{\mathrm{x}}=\mathrm{f}[\mathrm{x}, \mathrm{u}(\mathrm{t}), \mathrm{r}], \mathrm{x}\left(\mathrm{t}_{0}\right)=\mathrm{x}_{0}$

$y=h(x, r)$

donde $x=\left[I, s, m, T, V, \mu_{0}, \mu_{2}\right]^{T}, y=[\rho / \eta, T, v]^{T}, u=\left[i_{e}, q_{e}, q, T_{e}, T_{c}, m_{e}, s_{e}, \mu_{0_{e}}, \mu_{2 \varepsilon}\right]^{T}$ 
Los estados del reactor $(x)$ son la concentración del iniciador $(I)$, la concentración adimensional del solvente ( $s=S / S^{\circ}$, siendo $S$ y $S^{\circ}$ las concentraciones molares del solvente y del solvente puro), la concentración adimensional del monómero $\left(m=M / M^{\rho}\right.$, siendo $M$ y $M^{\rho}$ las concentraciones molares del monómero y del monómero puro), la temperatura ( $T$ ), el volumen ( $V$, y los momentos cero $\left(\mu_{0}\right)$ y dos $\left(\mu_{2}\right)$ de la distribución de pesos moleculares (DPM). El modelo del reactor depende de $n_{r}$ parámetros $r$.

Las entradas exógenas $(u)$ son la alimentación de iniciador $\left(i_{e}\right)$, el flujo de entrada $\left(q_{e}\right)$, el flujo de salida $(q)$, la temperatura de entrada $\left(T_{e}\right)$, la temperatura de la camisa de enfriamiento $\left(T_{c}\right)$, la concentración de monómero de entrada $\left(m_{e}\right)$, la concentración de solvente de entrada $\left(s_{e}\right)$ y las entradas de los momentos 0 y 2 de la DPM $\left(\mu_{0 e}\right.$ y $\left.\mu_{2 e}\right)$.

Las mediciones continuas instantáneas disponibles $(y)$ son la densidad (mediante un densitómetro Ellis y col., 1994-) y/o el índice de refracción (mediante un refractómetro - Schuler y Papadopolou, 1986-) como indicadores de la concentración de monómero; la temperatura (mediante un termopar) y el volumen (utilizando un medidor de nivel). Cuando se considera la medición simultánea de la densidad y el índice de refracción es posible el conocimiento de las concentraciones de monómero y solvente.

Los términos $r_{b}, r_{p}, r_{s o l}$ y $r_{p o l}$ son las tasas de descomposición del iniciador, consumo de monómero por propagación, consumo de solvente por transferencia y polimerización total respectivamente, y los términos $r_{\mu 0}$ y $r_{\mu 2}$ son las tasas de cambio de los momentos cero y dos de la DPM. Los términos $\beta$ y $\gamma$ son indicadores de la generación e intercambio de calor; $\varepsilon_{m}$ y $\varepsilon_{s}$ son factores de contracción debido a la diferencia de densidad entre el monómero y el polímero, y entre el solvente y el polímero, respectivamente. Los términos $r_{l}, r_{p}, r_{p o l}, r_{\text {sol }}, \beta$ y $\gamma$ son campos escalares suaves y estrictamente positivos en el espacio de estados del reactor. El modelo del reactor puede representar una operación de flujo continuo $\left[q_{e}=q+\left(\varepsilon_{m} r_{p o l}+\varepsilon_{s} r_{\text {sol }}\right) V, q \neq 0\right]$ de semilote $(q=0) 0$ de lote $\left(q_{\mathrm{c}}=\mathrm{q}=0\right)$. La exotermicidad de las reacciones de polimerización por radicales libres, unida al aumento de la viscosidad y la disminución de la capacidad de remoción de calor, origina que los reactores continuos manifiesten multiplicidad de estados estacionarios, y los reactores en lote o semilote presenten trayectorias de estado inestables. 


\section{Problema de estimación}

El problema de estimación del reactor consiste en la obtención de estimados de la conversión $(c)$, la fracción masa de sólidos $(\phi)$ el peso molecular en número $\left(M_{n}\right)$ y el peso molecular en peso $\left(M_{w}\right)$, variables que definen la velocidad de producción, la calidad del producto y la seguridad en la operación.

$$
\begin{aligned}
& c=\frac{1-m-s}{1-\varepsilon_{m} m-s} \\
& \phi=\frac{1-m-s}{1-\varepsilon_{m} m-\varepsilon_{s} s} \\
& M_{n}=\frac{\rho_{m}^{0}(1-m-s)}{\left(1-\varepsilon_{m}\right) \mu_{0}} \\
& M_{w}=\frac{P M_{m}^{2} \mu_{2}\left(1-\varepsilon_{m}\right)}{\rho_{m}^{0}(1-m-s)}
\end{aligned}
$$

donde $\rho_{\mathrm{m}}^{\circ}$ es la densidad del monómero puro y $P M_{m}$ es el peso molecular del monómero.

Una vez determinado el modelo que describe los estados de un reactor de homopolimerización y su problema de estimación, se procede al estudio de la resolubilidad del problema de estimación y construcción y sintonización de los estimadores. Para estos pasos de la metodología se establecerá previamente en el siguiente capítulo el marco de estimación no lineal. 


\section{Capítulo III. Estimación no lineal}

En este capítulo se presentarán las herramientas para el análisis de la resolubilidad del problema de estimación, la construcción y sintonización de estimadores geométricos no lineales. El diseño del esquema de estimación incluye dos etapas complementarias: una etapa de análisis donde el objetivo es la validación a priori de la resolubilidad del problema de estimación, y una etapa de síntesis en la que se realiza la construcción del estimador y las ganancias se sintonizan de acuerdo a un procedimiento sistemático. Una mayor profundidad de los detalles técnicos puede verse en Alvarez y López (1999).

\section{Problema de estimación}

Considérese la planta no lineal MIMO (Multiinput-multioutput):

$$
\begin{aligned}
& \dot{x}=f[x, u(t), r], x\left(t_{0}\right)=x_{0} \\
& y=h(x, r) \\
& x_{0} \in X_{0}, \quad x \in X, \quad r \in R, u \in U
\end{aligned}
$$

con $n$ estados $(x), m$ salidas medidas $(y), p$ entradas exógenas conocidas $(u)$, y $n_{r}$ parámetros del modelo $(r) . X_{0}$ es el conjunto de admisibles estados iniciales. Las entradas $u$, los estados $x$ y los parámetros $r$ toman valores en los conjuntos $U, X$, y $R$, los cuales son compactos (acotados y cerrados) debido a consideraciones prácticas y físicas. Los mapas $f$ y $h$ son suficientemente suaves (diferenciables) en sus respectivos dominios.

Cada conjunto $\left\{\mathrm{x}_{0}, \mathrm{u}(\mathrm{t}), \mathrm{r}\right\}$ determina de manera única una trayectoria de estado (posiblemente inestable) $x(t)=\theta_{x}\left[t, t_{0}, x_{0}, u(t), r\right]$ que a su vez determina una trayectoria de salida $y(t)=h[x(t), r]$.

La trayectoria $x(t)$ de (3.1) se denomina RE (robusta y exponencialmente) - estable si existen cuatro constantes $a_{x}, \lambda_{x}, b_{w}$, y $b_{r}>0$ tal que, en una vecindad en forma de "tubo" de $x(t)$, la trayectoria perturbada

$\chi(t)=\theta_{x}\left[t, t_{0}, \chi_{0}, \psi_{u}(t), p\right]$ 
converge como sigue:

$$
\begin{aligned}
& \|\chi(t)-x(t)\| \leq a_{x} e^{-\lambda_{x}\left(t-t_{0}\right)}\left\|\chi_{0}-x_{0}\right\|+b_{u}\left\|\Psi_{u}(t)-u(t)\right\|^{s}+b_{r}\|\rho-r\| \\
& \text { donde }\left\|\Psi_{u}(t)-u(t)\right\|^{s}={ }_{i} \text { sup }\left\|\Psi_{u}(t)-u(t)\right\|
\end{aligned}
$$

El problema de estimación consiste en el diseño de un estimador dinámico de estados

$$
\begin{aligned}
& \dot{x}_{e}=\mathrm{f}\left[\chi_{e}, \mathrm{u}(\mathrm{t}), \mathrm{y}(\mathrm{t}), \rho\right], \quad \chi_{e}\left(\mathrm{t}_{0}\right)=\chi_{\mathrm{e}} \\
& \chi=\mathrm{h}_{\mathrm{e}}\left(\chi_{\mathrm{e}}, \rho\right)
\end{aligned}
$$

construido con una aproximación $\rho$ de los parámetros $r$, tal que, dirigido por las señales medidas $u(\mathrm{t})$ y $y(\mathrm{t})$, genere una trayectoria inferida $\chi(\mathrm{t})$ que converja $\mathrm{RE}$ (robusta y exponencialmente) a la trayectoria de la planta $x(t)$.

Antes de proceder al establecimiento del estimador (3.3) de la planta no lineal (3.1), primero se debe verificar que la trayectoria $x(t)$ sea estimable, para lo cual se utilizará la definición de estimabilidad de Alvarez y López (1999).

\section{Estimabilidad}

Como un paso metodológico para la definición de la propiedad de estimabilidad y con base en la prueba de suficiencia de la condición de observabilidad para plantas lineales (Kwakernaak y Sivan, 1972), se considerará un problema auxiliar: la determinación (con unicidad) del estado de la planta $x(t)$ en base a los parámetros conocidos $r$, las señales medidas $u(t)$ y $y(t)$ y sus derivadas temporales.

La solución de este problema auxiliar consta de dos pasos:

1. Obtención de un conjunto de ecuaciones algebraicas independientes variantes en tiempo y bien condicionadas, donde la incógnita sea el estado de planta.

Para la obtención de tales ecuaciones, sea la derivada direccional del campo escalar $\alpha(\mathrm{x}, \mathrm{t})$ en dirección del campo vectorial $f(x, t)$ :

$$
L_{f} \alpha=\sum_{i=1}^{n} f_{i}(x) \frac{\partial \alpha}{\partial x_{i}}+\frac{\partial \alpha}{\partial t}
$$

y su fórmula recursiva:

$$
\mathrm{L}_{\mathrm{f}}^{\mathrm{i}+1} \alpha=\mathrm{L}_{\mathrm{f}}\left(\mathrm{L}_{\mathrm{f}}^{\mathrm{i}} \alpha\right) \quad \mathrm{L}_{\mathrm{f}}^{0} \alpha=\alpha
$$

Aplicando (3.4) al mapa de salida (3.1b) $\left(\kappa_{i}+1\right)$ veces a cada una de las $i$ salidas $(i=1, \ldots, m)$, se 
obtendrán $(\mathrm{K}+\mathrm{m})$ ecuaciones algebraicas, dependientes del tiempo:

$$
Y(t)=\Phi_{1}[x, \mathcal{U}(\mathrm{t}), r], \quad Y(t)=\varphi[\mathrm{x}, \mathcal{U}(\mathrm{t}), \mathrm{r}]
$$

donde

$$
\begin{aligned}
& Y(t)=\left[y_{1}, \ldots, y_{1}^{\left(x_{i}-1\right)} ; \ldots ; y_{m}, \ldots, y_{m}^{\left(x_{m}-1\right)}\right]^{T} \\
& Y(t)=\left[y_{1}^{\left(x_{1}\right)}, \ldots, y_{1}^{\left(x_{m}\right)}\right]^{T}
\end{aligned}
$$

$\kappa_{1}+\ldots+\kappa_{m}=\kappa \leq n$

$\kappa_{\mathbf{i}}>0$

$U(t)=\left[u_{1}, \ldots, u_{1}^{\left(v_{1}-1\right)} ; \ldots ; u_{p}, \ldots, u_{p}^{\left(v_{p}-1\right)}\right]^{T}$

$v_{1}+\ldots+v_{p}=v \geq p$

$v_{\mathrm{i}}>0$

$\Phi_{1}\left(\mathrm{x}, U_{,} \mathrm{r}\right)=\left[\mathrm{h}_{1}, \ldots, \mathrm{L}_{\mathrm{f}}^{\mathrm{r}_{1}-1} \mathrm{~h}_{1} ; \ldots ; \mathrm{h}_{\mathrm{m}}, \ldots, \mathrm{L}_{\mathrm{f}}^{\mathrm{x}_{\mathrm{m}}-1} \mathrm{~h}_{\mathrm{m}}\right]^{\mathrm{T}}$

$\varphi[x, \mathcal{U}(\mathrm{t}), r]=\left[\mathrm{L}_{\mathrm{f}}^{\mathrm{x}_{1}} \mathrm{~h}_{1}, \ldots, \mathrm{L}_{\mathrm{f}}^{\mathrm{x}_{\mathrm{m}}} \mathrm{h}_{\mathrm{m}}\right]^{\mathrm{T}}$

Si (3.6a) consta de $\kappa$ ecuaciones independientes y $\kappa=n$, para cada $t$ existe solución única para el estado de la planta:

$$
x(t)=\Phi_{1}^{-1}[Y(t), \mathcal{U}(t), r]
$$

En caso de que $\kappa<n$, la solución $x(t)$ se encuentra en la hipersuperficie de dimensión $(n-k)$ :

$\Xi(t)=\left\{x \in X \mid \Phi_{I}[x, U(t), r]=Y(t)\right\}$

que representa la intersección de las $\kappa$ hipersuperficies de dimensión $(n-l)$ descritas por las $\kappa$ ecuaciones algebraicas (3.6a) dependientes del tiempo.

2. Obtención de un conjunto de $(\boldsymbol{n}-\boldsymbol{\kappa})$ ecuaciones adicionales. En el caso de que $\boldsymbol{k}<\boldsymbol{n}$ se puede resolver para los estados $x_{\mathrm{I}},\left(\operatorname{dim} \mathrm{x}_{\mathrm{I}}=\kappa\right)$ en función de los estados $\mathrm{x}_{\mathbb{I}}\left(\operatorname{dim} \mathrm{x}_{\mathbb{I}}=\mathbf{n}-\boldsymbol{\kappa}\right)$ :

$$
x_{1}(t)=\Phi_{1}^{-1}\left[x_{n}, Y(t), \mathcal{U}(t), r\right]
$$

la planta (3.1a) puede expresarse:

$$
\begin{aligned}
& \dot{x}_{I}=f_{I}\left[x_{1}, x_{I I}, u(t), r\right] \\
& \dot{x}_{I I}=f_{I I}\left[x_{1}, x_{I I}, u(t), r\right]
\end{aligned}
$$


dado que la solución para $x_{I}(t)$ está representada por (3.10), el sistema (3.11) se expresa como

$$
\begin{aligned}
& x_{I}(t)=\Phi_{I}^{-1}\left[x_{I I}, Y(t), U(t), r\right] \\
& \dot{x}_{I I}=f_{I}\left\{\Phi_{I}^{-1}\left[x_{I I}, Y(t), U(t), r\right], x_{I I}, u(t), r\right\}=f^{*}\left[x_{I I}, Y(t), U(t), r\right]=\dot{x}^{*}
\end{aligned}
$$

A (3.12a) se le denomina estados de la dinámica observable, mientras que (3.12b) es la dinámica no observable. $\Xi(\mathrm{t})$ se denomina superficie no observable en tanto que, a menos que $\kappa=n$, de la ecuación (3.6a) no se puede decir en donde se encuentra $x(t)$ en $\Xi(t)$. La planta $x(t)$ y su trayectoria reconstruida $\chi(t)$ coinciden, pero sus propiedades de estabilidad no son necesariamente las mismas. Debido a que $\chi(t)$ es la restricción de $x(t)$ a la superficie no observable $\Xi(t), y \chi(t)$ tiene la propiedad de estabilidad de $x^{*}(t)$, entonces: si $x(t)$ es $R$ (robustamente)-estable, $x^{*}(t)$ es $R$ estable; si $x(t)$ es $N$ (nominalmente)-estable, $x^{*}(t)$ es N-estable o R-estable; y si $x(t)$ es inestable, $\mathbf{x}^{*}(t)$ es inestable, $\mathrm{N}$-estable o R-estable.

Definición. La trayectoria $x(t)$ de la planta (3.1) es RE (robusta y exponencialmente) estimable si existen $m$ enteros (índices de observabilidad) $\kappa_{1}, \ldots, \kappa_{m}\left(\kappa_{l}+\ldots+\kappa_{m}=\kappa \leq n, \kappa_{s}>0\right)$ y un mapa $\Phi_{\mathrm{II}}(\mathrm{x}$, $U, r)=\left[\phi_{k+1}, \ldots, \phi_{n}\right]^{\top}$ tal que, en una vecindad alrededor de $\{x(t), U(t), r\}$ (la trayectoria de la planta, la trayectoria de las entradas y sus derivadas, y los parámetros):

(i) El mapa $\Phi\left(\mathrm{x}, U_{,} \mathrm{r}\right)=\left[\begin{array}{l}\Phi_{1} \\ \Phi_{\mathrm{II}}\end{array}\right]$ es $\mathrm{R}$ (robustamente) - invertible para $\mathrm{x}$.

(ii) El mapa $\varphi(x, U, r)=\left[L_{f}^{k_{i}} h_{1} ; \ldots ; L_{f}^{k_{n}} h_{m}\right]^{T}$ es $L$ (Lipschitz) - continuo (continuamente diferenciable)

(iii) La trayectoria no observable $\mathrm{x}^{*}(\mathrm{t})$ es $\mathrm{RE}$ (robusta y exponencialmente) - estable.

Si $\kappa=n$, (es decir, no existe dinámica no observable), la trayectoria $x(t)$ es RE - observable; de otra forma, si $\kappa<n$, la trayectoria es RE - detectable. Los enteros $v_{1}, \ldots, v_{p}\left(v_{p}>0\right)$ se denominan indices de exo - observabilidad. 


\section{Estimador no lineal}

Si la trayectoria $x(t)$ de la planta es RE - estimable en el sentido de la definición de estimabilidad, el estimador no lineal es de la forma $\left(\Gamma_{u}, y \Delta_{u}\right.$ son matrices diagonales en bloques definidas en el apéndice $\mathrm{A}$ ):

$$
\begin{aligned}
& \dot{\chi}_{u}=\Gamma_{u} \chi_{u}+K_{u}\left(s_{u}\right)\left(u-\Delta_{u} \chi_{u}\right) \\
& \psi_{u}=\Delta_{u} \chi_{u} \\
& \dot{\chi}=f\left[\chi, \Delta_{u} \chi_{u}, \rho\right]+G_{p}\left(\chi, \chi_{u}, \rho\right)[y-h(\chi, \rho)]+G_{1}\left(x, \chi_{u}, \rho\right) \downarrow_{a}[y-h(x, \rho)] d \tau \\
& \psi=h(\chi, \rho)
\end{aligned}
$$

Designando a $U$ (entradas exógenas y sus derivadas temporales) como un vector de estados $x_{u}$ de un exosistema dinámico, su aproximación es $\chi_{u}$. En (3.13c) el primer término del lado derecho es la aproximación basada en el modelo de la velocidad de cambio de los estados, el segundo y tercer término son correcciones por acción proporcional e integral, respectivamente. La acción integral ayuda a reducir el sesgo de estimación causado por errores en el modelado. El estimador (3.13) tiene como ganancias proporcionales $\left(K_{u}, K_{o}\right)$ e integral $\left(K_{l}\right)$ parametrizadas:

$$
\begin{aligned}
& K_{u}\left(s_{u}\right)=b d\left[\left(s_{u} k_{11}^{u}, \ldots, s_{u}^{v_{v}} k_{v_{1}}^{u}\right)^{T}, \ldots,\left(s_{u} k_{1 p}^{u}, \ldots, s_{u}^{v} \cdot k_{v_{p} p}^{u}\right)^{T}\right] ; \quad s_{u}>0 \\
& K_{0}\left(s_{0}\right)=b d\left[\left(s_{0} k_{11}^{0}, \ldots, s_{0}^{k_{1}} k_{k_{1} 1}^{0}\right)^{T}, \ldots,\left(s_{0} k_{1 m}^{0}, \ldots, s_{0}^{k_{1}}-k_{k_{m}}^{0}\right)^{T}\right] ; \quad s_{0}>0 \\
& K_{I}\left(s_{0}\right)=\operatorname{diag}\left(s_{0}^{K_{1}+1} k_{1}^{1}, \ldots, s_{0}^{k_{n}+1} k_{m}^{1}\right)
\end{aligned}
$$

cuyos valores de $K_{u}(1), K_{o}(1)$ y $K_{C}(1)$ estabilizan las dinámicas lineales, no interactivas y de polo asignable (LNPA) de los errores de salida:

$$
\begin{array}{lll}
\mu_{i}^{\left(v_{i}\right)}+k_{1 i}^{u} \mu_{i}^{\left(v_{i}-1\right)}+\ldots+k_{v_{i}}^{u} \mu_{i}=0 ; & \mu_{i}=\psi_{i}^{u}-u_{i} \\
v_{i}^{\left(k_{i}+1\right)}+k_{1 i}^{0} v_{i}^{\left(k_{i}\right)}+\ldots+k_{k_{i} i}^{0} v_{i}^{(1)}+k_{i}^{1} v_{i}=0 ; & v_{i}=\psi_{i}-y_{i}
\end{array}
$$

Las matrices no lineales $G_{p}$ y $G_{l}$ en (3.13c) son $\left(\Pi_{0}\right.$ definida en el apéndice A):

$$
\begin{aligned}
& G_{p}\left(\chi, \chi_{u}, \rho\right)=\Theta_{1}\left(\chi, \chi_{u}, \rho\right) K_{o}\left(s_{o}\right) \\
& G_{1}\left(\chi, \chi_{u}, \rho\right)=\Theta_{1}\left(\chi, \chi_{u}, \rho\right) \Pi_{o} K_{1}\left(s_{o}\right) \\
& {\left[\Theta_{1}, \Theta_{I 1}\right]=\Phi_{x}^{-1}\left(\chi, \chi_{u}, \rho\right) \quad \operatorname{dim} \Theta_{1}=\kappa ; \operatorname{dim} \Theta_{\| 1}=n-\kappa}
\end{aligned}
$$


donde $\Phi_{\mathrm{x}}^{-1}$ es la inversa del jacobiano del mapa $\Phi$.

Cuando $\kappa=n$, mediante la sintonización de los parámetros $s_{u}$ y $s_{o}$ de forma suficientemente grande: (i) las salidas y los estados pueden converger arbitrariamente rápidos, (ii) el sesgo de las salidas puede hacerse arbitrariamente pequeño, y (iii) la magnitud del sesgo de los estados puede hacerse arbitrariamente cercana hacia un valor límite. Cuando $\kappa<n$, mediante la sintonización de los parámetros $s_{u}$ y $s_{o}$ de forma suficientemente grande: (i) las salidas pueden converger arbitrariamente rápido, (ii) el sesgo de las salidas puede hacerse arbitrariamente pequeño, (iii) los estados pueden converger con una velocidad arbitrariamente cercana a la de la trayectoria del error no observable, y (iv) la magnitud del sesgo de los estados puede hacerse arbitrariamente cercana hacia un valor límite. Estas propiedades de convergencia identifican las capacidades y limitaciones del desempeño del estimador. La acción integral contribuye a que se reduzcan los sesgos en la estimación de estados y salidas provocados por los errores de modelado, pero la convergencia es más lenta y oscilatoria. En su implantación, estas capacidades estarán limitadas por la presencia de ruido en las mediciones y errores numéricos y de modelado.

\section{Sintonización}

Para confrontar el problema de divergencia en la estimación o convergencia con deficiente robustez (lo que ocurre en sistemas con dinámicas altamente no lineales) se presentará un método de sintonización mediante colocación de polos.

Sean la dinámica LNPA del error de estimación de la salida $i$ para el estimador no lineal:

$$
v_{i}^{\left(k_{i}+1\right)}+k_{1 i}^{0} v_{i}^{\left(k_{i}\right)}+\ldots+k_{k_{i} i}^{0} v_{i}^{(1)}+k_{k_{i}+1, i}^{I} v_{i}=0
$$

y reescribase de la siguiente forma:

$$
v_{i}^{\left(n_{i}\right)}+k_{i}^{i} v_{i}^{\left(n_{i}-1\right)}+\ldots+k_{n_{i}-1}^{i} v_{i}^{(1)}+k_{n_{i}}^{i} v_{i}=0
$$

donde $n_{i}=\kappa_{i}+1\left(n_{i} \geq 2\right)$. El polinomio caracteristico de (3.18) es:

$$
\begin{aligned}
& \left(\gamma_{i}\right)^{n_{i}}+k_{1}^{i}\left(\gamma_{i}\right)^{n_{i}-1}+\ldots+k_{n_{i}-1}^{i} \gamma_{i}+k_{n_{i}}^{i}=0 \\
& =\left(\gamma_{i}-\gamma_{1}^{i}\right)\left(\gamma_{i}-\gamma_{2}^{i}\right) \ldots\left(\gamma_{i}-\gamma_{n_{i}}^{i}\right)
\end{aligned}
$$

El polinomio característico (3.19) tiene $n_{i}$ polos de referencia $\gamma_{1}^{i}, \ldots, \gamma_{n_{i}}^{i}$, los cuales estabilizarán la dinámica del error (3.18) si tales polos se encuentran a la izquierda en el plano complejo. Si $n_{i}$ es 
un número par, la dinámica LNPA tendrá $n_{\sqrt{ }} / 2$ pares repetidos de polos complejos conjugados con frecuencia caracteristica de referencia $\omega_{2}^{i}$ (frecuencia para una dinámica de $2^{\circ}$ orden) y un factor de amortiguamiento suficientemente grande $\left(\zeta_{>}>0.71\right)$ para prevenir comportamiento excesivamente oscilatorio. Si $n_{i}$ es impar, $n_{i}$ - 1 polos se asignan de la misma forma, y el $n_{i}$-iésimo polo es un número real igual a $\omega_{1}^{i}$ (frecuencia para una dinámica de $1^{\text {er }}$ orden). Esto es, para $s_{o}=1$ :

$\gamma_{j, j+1}^{\mathrm{i}}(1)=-\omega_{2}^{\mathrm{i}}\left(\zeta_{\mathrm{i}} \mp \sqrt{\zeta_{\mathrm{i}}^{2}-1}\right)$

$\mathrm{i}=1, \ldots, \mathrm{m}$

$j=1,3,5, \ldots, n_{i}-1\left(n_{i}\right.$ par) o $n_{i}-2\left(n_{i}\right.$ impar $)$

$\gamma_{\mathrm{n}_{\mathrm{i}}}^{\mathrm{i}}(1)=-\omega_{1}^{\mathrm{i}}$

solo para $n_{i}$ impar

A partir de la comparación entre (3.19) y las dinámicas de $1^{\circ}$ y $2^{\circ}$ expresadas en términos de la frecuencia (o bien el tiempo de asentamiento $\tau_{a}$ ) y el factor de amortiguamiento $(\zeta)$ (Stephanopoulos, 1984), se obtiene la expresión para las ganancias $k_{1 i}^{\circ}, \ldots, k_{\mathrm{x}_{\mathrm{i}}}^{\circ}, \mathbf{k}_{\mathrm{i}}^{\mathrm{I}}$.

Así, para una dinámica de 20 . orden $\left(n_{i}=2\right)$ :

$v_{i}^{(2)}+k_{1}^{i} v_{i}^{(1)}+k_{2}^{i} v_{i}=0$

las ganancias son:

$$
k_{1}^{i}=\frac{8}{\left(\tau_{a}\right)_{i}}, \quad k_{2}^{i}=\frac{16}{\left(\tau_{a} \xi\right)_{i}^{2}}
$$

Para una dinámica de 3er. orden $\left(n_{i}=3\right)$ :

$v_{i}^{(3)}+k_{1}^{i} v_{i}^{(2)}+k_{2}^{i} v_{i}^{(1)}+k_{3}^{i} v_{i}=0$

las ganancias son:

$$
k_{1}^{i}=\frac{12}{\left(\tau_{\mathrm{a}}\right)_{i}}, \quad k_{2}^{i}=\frac{16}{\left(\tau_{\mathrm{a}}\right)_{i}^{2}}\left[2+\frac{1}{(\xi)_{i}^{2}}\right], \quad k_{3}^{i}=\frac{64}{(\xi)_{i}^{2}\left(\tau_{\mathrm{a}}\right)_{i}^{3}}
$$

En resumen, la definición de estimabilidad proporciona una herramienta para determinar tal propiedad de una trayectoria de la planta; la elección del conjunto de índices de observabilidad constituye un grado de libertad en el diseño del estimador. Realizado el análisis de estimabilidad, la construcción y sintonización del estimador no lineal son procedimientos sistemáticos. 


\section{Capítulo IV. Resolubilidad del problema de estimación}

En este capitulo se realizará la identificación de los conjuntos de índices de observabilidad posibles para cada conjunto de mediciones, de acuerdo con la física del problema y aspectos de ingeniería relacionados. Con cada conjunto de índices de observabilidad se verificará el cumplimiento de las tres condiciones de la definición de estimabilidad (Alvarez y Lopez, 1999). A partir del cumplimiento de las tres condiciones se concluirá si la trayectoria de los estados es RE estimable con un conjunto de índices dado; como prueba inicial cualitativa de robustez se considerará la eliminación de los factores de contracción, en la idea de que en una situación industrial no se detectan con gran sensibilidad los cambios de nivel en un reactor. Cada condición de la definición de estimabilidad se identificará como sigue:

(i) Invertibilidad robusta (para $\mathrm{x}$ ) del mapa de estimabilidad $\Phi$.

(ii) Continuidad Lipschitz del mapa $\varphi$.

(iii) Estabilidad robusta y exponencial de la dinámica no observable $\left(x_{I I}\right)$.

\section{Mediciones: concentración de monómero, temperatura y volumen}

Aunque en un proceso real no se dispone de la concentración de monómero como medición, se consideró ésta en primer término para tener un referente inicial para el estudio con mediciones directas de densidad e índice de refracción. La concentración de monómero $(m)$ es conocida a través del empleo de un densitómetro (Ellis y col.,1994) o de un medidor de índice de refracción (Shuler y Papadopolou, 1986). Las mediciones a estudiar son.

$\mathrm{y}=[\mathrm{m}, \mathrm{T}, \mathrm{v}]^{\mathrm{T}}=\left[\mathrm{x}_{3}, \mathrm{x}_{4}, \mathrm{x}_{5}\right]^{\mathrm{T}}$

donde $m$ es la concentración de monómero (adimensional). De la definición de estimabilidad y del reactor (2.2), para cualquier conjunto de índices de observabilidad $k=\left\{\kappa_{m}, \kappa_{\mathrm{T}}, \kappa_{v}\right\}$ se obtiene el siguiente conjunto de ecuaciones que abarca las mediciones y sus derivadas temporales; tal conjunto de ecuaciones es independiente de los momentos 0 y $2\left(\mu_{0}\right.$ y $\left.\mu_{2}\right)$,:

$\mathrm{y}_{1}=\mathrm{m}$

$\dot{\mathrm{y}}_{\mathrm{l}}=\dot{\mathrm{m}}=\mathrm{f}_{\mathrm{m}}(\mathrm{I}, \mathrm{s}, \mathrm{m}, \mathrm{T}, \mathrm{V})$ 


$$
\begin{aligned}
& \ddot{y}_{1}=\frac{\partial f_{m}}{\partial I} \dot{I}+\frac{\partial f_{m}}{\partial s} s+\frac{\partial f_{m}}{\partial m} \dot{m}+\frac{\partial f_{m}}{\partial T} \dot{T}+\frac{\partial f_{m}}{\partial V} \dot{V} \\
& =\frac{\partial f_{m}}{\partial I}(I, s, m, T) f_{I}(I, s, m, T, V)+\frac{\partial f_{m}}{\partial s}(I, s, m, T) f_{s}(I, s, m, T, V) \\
& +\frac{\partial f_{m}}{\partial m}(I, s, m, T, V) f_{m}(I, s, m, T, V)+\frac{\partial f_{m}}{\partial T}(I, s, m, T) f_{T}(I, s, m, T, V) \\
& +\frac{\partial f_{m}}{\partial V}(m, V) f_{v}(I, s, m, T, V) \\
& \vdots \\
& \mathrm{y}_{2}=\mathrm{T} \\
& \dot{\mathrm{y}}_{2}=\dot{\mathrm{T}}=\mathrm{f}_{\mathrm{T}}(\mathrm{I}, \mathrm{s}, \mathrm{m}, \mathrm{T}, \mathrm{V}) \\
& \ddot{y}_{2}=\frac{\partial f_{\mathrm{T}}}{\partial \mathrm{I}} \dot{\mathbf{I}}+\frac{\partial \mathrm{f}_{\mathrm{T}}}{\partial \mathrm{s}} \dot{\mathbf{s}}+\frac{\partial \mathrm{f}_{\mathrm{T}}}{\partial \mathrm{m}} \dot{\mathrm{m}}+\frac{\partial \mathrm{f}_{\mathrm{T}}}{\partial \mathrm{T}} \dot{\mathrm{T}}+\frac{\partial \mathrm{f}_{\mathrm{T}}}{\partial \mathrm{V}} \dot{\mathrm{V}} \\
& =\frac{\partial f_{\mathrm{T}}}{\partial \mathrm{I}}(\mathrm{I}, \mathrm{s}, \mathrm{m}, \mathrm{T}) \mathrm{f}_{\mathrm{I}}(\mathrm{I}, \mathrm{s}, \mathrm{m}, \mathrm{T}, \mathrm{V})+\frac{\partial \mathrm{f}_{\mathrm{T}}}{\partial \mathrm{s}}(\mathrm{I}, \mathrm{s}, \mathrm{m}, \mathrm{T}, \mathrm{V}) \mathrm{f}_{\mathrm{s}}(\mathrm{I}, \mathrm{s}, \mathrm{m}, \mathrm{T}, \mathrm{V}) \\
& +\frac{\partial f_{T}}{\partial m}(I, s, m, T, V) f_{m}(I, s, m, T, V)+\frac{\partial f_{T}}{\partial T}(I, s, m, T, V) f_{T}(I, s, m, T, V) \\
& +\frac{\partial f_{T}}{\partial V}(s, m, T, V) f_{v}(I, s, m, T, v) \\
& \vdots \\
& \mathrm{y}_{3}=\mathrm{V} \\
& \dot{\mathrm{y}}_{3}=\dot{\mathrm{V}}=\mathrm{f}_{\mathrm{v}}(\mathrm{I}, \mathrm{s}, \mathrm{m}, \mathrm{T}, \mathrm{V}) \\
& \ddot{y}_{3}=\frac{\partial f_{V}}{\partial I} \dot{I}+\frac{\partial f_{V}}{\partial s} \dot{s}+\frac{\partial f_{V}}{\partial m} \dot{m}+\frac{\partial f_{v}}{\partial T} \dot{T}+\frac{\partial f_{V}}{\partial V} \dot{V} \\
& =\frac{\partial f_{V}}{\partial I}(I, s, m, T, V) f_{i}(I, s, m, T, V)+\frac{\partial f_{V}}{\partial s}(I, s, m, T, V) f_{s}(I, s, m, T, V) \\
& +\frac{\partial f_{v}}{\partial m}(I, s, m, T, V) f_{m}(I, s, m, T, V)+\frac{\partial f_{v}}{\partial T}(I, s, m, T, V) f_{T}(I, s, m, T, V) \\
& +\frac{\partial f_{v}}{\partial V}(I, s, m, T, V) f_{v}(I, s, m, T, V)
\end{aligned}
$$

$\vdots$

por lo que el mapa de estimabilidad $\Phi_{1}$ :

$\Phi_{1}(\mathrm{x}, U, \mathrm{r})=\left[\mathrm{m}, \ldots, \mathrm{m}^{\left(\mathrm{x}_{\mathrm{m}}-1\right)}, \mathrm{T}, \ldots, \mathrm{T}^{\left(\mathrm{k}_{\mathrm{r}}-1\right)}, \mathrm{V}, \ldots, \mathrm{v}^{\left(\mathrm{k}_{\mathrm{v}}-1\right)}\right]^{\mathrm{T}}$

es independiente de los momentos y entonces estos estados son no observables. La dimensión del mapa no observable $\Phi_{I I}$ es $(n-\kappa) \geq 2$. Por tanto, el entero $\kappa=\kappa_{m}+\kappa_{T}+\kappa v$ puede ser 3, 4 o 5, con las siguientes posibilidades: 


\begin{tabular}{|c|c|}
\hline $\begin{array}{c}\text { Grupo } \\
\kappa=\kappa_{\mathrm{m}}+\kappa_{\mathrm{T}}+\kappa_{\mathrm{V}}\end{array}$ & $\begin{array}{c}\text { Conjuntos posibles } \\
k=\left\{\boldsymbol{K}_{\mathrm{m}}, \kappa_{\mathrm{T}}, \kappa_{\mathrm{V}}\right\}\end{array}$ \\
\hline$\kappa=3$ & $\{1,1,1\}$ \\
\hline$\kappa=4$ & $\{2,1,1\},\{1,2,1\},\{1,1,2\}$ \\
\hline$\kappa=5$ & $\{3,1,1\},\{1,3,1\},\{1,1,3\}$ \\
& $\{2,2,1\},\{2,1,2\},\{1,2,2\}$ \\
\hline
\end{tabular}

Grupo $\kappa=3$, conjunto $\{1,1,1\}$

(i) Siendo el mapa de observabilidad $\Phi_{I}$ y el mapa $\Phi_{I I}$ de estados no observables los siguientes:

$\Phi_{1}=\left[\begin{array}{lll}m & T & V\end{array}\right]^{\top}$

$\Phi_{\mathrm{II}}=\left[\begin{array}{llll}1 & \mathrm{~s} & \mu_{0} & \mu_{2}\end{array}\right]^{\mathrm{T}}$

el mapa de estimabilidad $\Phi$ es

$\Phi=\left[\begin{array}{lllllll}m & T & V & I & s & \mu_{0} & \mu_{2}\end{array}\right]^{T}$

el cual es trivialmente $\mathrm{x}$-invertible (invertible para el vector de estados $\boldsymbol{x}$ ).

(ii) El mapa $\varphi$ es L-continuo (continuamente diferenciable):

$\varphi=\left[\begin{array}{lll}f_{m} & f_{T} & f_{v}\end{array}\right]^{T}$

La continuidad Lipschitz se verifica para el caso de polimerización en masa (Alvarez y López, 1999) y los términos adicionales en las ecuaciones diferenciales son la contribución de transferencia a solvente y la concentración de entrada del mismo; la continuidad Lipschitz se cumple para la polimerización en solución.

(iii) La dinámica no observable es RE-estable:

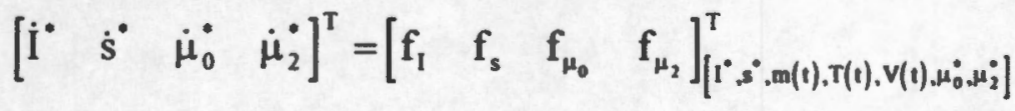

Las trayectorias de la dinámica no observable son RE-estables debido a que la dinámica del monómero no se encuentra entre las no observables. La dinámica del monómero con efecto gel es la única fuente de inestabilidad de las trayectorias del reactor. 
Grupo $\kappa=4$, conjuntos $\{2,1,1\},\{1,2,1\},\{1,1,2\}$

(i) Los mapas de observabilidad respectivos son

$$
\Phi_{1}=\left[\begin{array}{llll}
m & f_{m} & T & V
\end{array}\right]^{\mathrm{T}}, \Phi_{1}=\left[\begin{array}{llll}
m & T & f_{T} & V
\end{array}\right]^{T}, \Phi_{1}=\left[\begin{array}{llll}
m & T & V & f_{V}
\end{array}\right]^{T}
$$

se proponen como estados no observables

$$
\Phi_{11}=\left[\begin{array}{lll}
s & \mu_{0} & \mu_{2}
\end{array}\right]^{\mathrm{T}}
$$

con lo que los mapas de estimabilidad son

$$
\begin{aligned}
& \Phi=\left[\begin{array}{lllllll}
m & f_{m} & T & V & s & \mu_{0} & \mu_{2}
\end{array}\right]^{T} \\
& \Phi=\left[\begin{array}{lllllll}
m & T & f_{T} & V & s & \mu_{0} & \mu_{2}
\end{array}\right]^{T} \\
& \Phi=\left[\begin{array}{llllllll}
m & T & V & f_{V} & s & \mu_{0} & \mu_{2}
\end{array}\right]^{T}
\end{aligned}
$$

De las siguientes propiedades fisicas: aumento de la tasa de polimerización con la concentración de iniciador $\left(\partial \mathrm{r}_{\mathrm{pol}} / \partial \mathrm{I}>0\right)$, aumento de la tasa de transferencia a solvente con la concentración de iniciador $\left(\partial \mathrm{r}_{\text {sol }} / \partial \mathrm{I}>0\right)$ y mayor dependencia de la tasa de polimerización con el iniciador que la tasa de transferencia a solvente con el mismo $\left(\partial \mathrm{r}_{\mathrm{pol}} / \partial \mathrm{I}>\partial \mathrm{r}_{\mathrm{sol}} / \partial \mathrm{I}\right)$; y del modelo del reactor se observa que los mapas del conjunto $\left\{\mathrm{f}_{\mathrm{m}}, \mathrm{f}_{\mathrm{T}}, \mathrm{f}_{\mathrm{v}}\right\}$ dependen de la concentración de iniciador:

$$
\begin{aligned}
& \frac{\partial f_{m}}{\partial I}=-\left(1-\varepsilon_{m} m\right) \frac{\partial r_{p o l}}{\partial I}+\varepsilon_{s} m \frac{\partial r_{s o l}}{\partial I}<0 \\
& \frac{\partial f_{T}}{\partial I}=\beta(m, s) \frac{\partial r_{p}}{\partial I}>0 \\
& \frac{\partial f_{V}}{\partial I}=-\left(\varepsilon_{m} \frac{\partial r_{p o l}}{\partial I}+\varepsilon_{s} \frac{\partial r_{s o l}}{\partial I}\right) V<0
\end{aligned}
$$

por lo que cada mapa del conjunto $\left\{\mathrm{f}_{\mathrm{m}}, \mathrm{f}_{\mathrm{T}}, \mathrm{f}_{\mathrm{v}}\right\}$ es $I$-invertible, y los 3 mapas $\Phi$ son $x$-invertibles. Realizando el análisis de estimabilidad robusta (despreciando factores de contracción), se obtiene:

$$
\frac{\partial \mathrm{f}_{\mathrm{m}}}{\partial \mathrm{I}} \approx-\frac{\partial \mathrm{r}_{\mathrm{pol}}}{\partial \mathrm{I}}<0, \quad \frac{\partial \mathrm{f}_{\mathrm{T}}}{\partial \mathrm{I}}=\beta(\mathrm{m}, \mathrm{s}) \frac{\partial \mathrm{r}_{\mathrm{p}}}{\partial \mathrm{I}}>0, \quad \frac{\partial \mathrm{f}_{\mathrm{v}}}{\partial \mathrm{I}} \approx 0
$$

con lo que se observa que cada mapa del conjunto $\left\{\mathrm{f}_{\mathrm{m}}, \mathrm{f}_{\mathrm{T}}\right\}$ es $\mathrm{Rl}$-invertible y los mapas $\Phi$ correspondientes $(\{2,1,1\}$ y $\{1,2,1\})$ son $R x$-invertibles. El mapa $f_{V}$ se iguala a cero, con lo que el correspondiente mapa $\Phi$ no es $R x$-invertible y se descarta $\{1,1,2\}$ para la estimabilidad robusta. 
(ii) Los mapas $\varphi$ (para $\{2,1,1\}$ y $\{1,2,1\}$ ) son L-continuos:

$$
\varphi=\left[\begin{array}{lll}
L_{f} f_{m} & f_{T} & f_{v}
\end{array}\right]^{T}, \varphi=\left[\begin{array}{lll}
f_{m} & L_{f} f_{T} & f_{v}
\end{array}\right]^{T}
$$

(iii) La dinámica no observable es RE-estable:

$\left.\left[\begin{array}{lll}\dot{s}^{*} & \ddot{\mu}_{0}^{*} & \ddot{\mu}_{2}^{*}\end{array}\right]^{T}=\left[\begin{array}{lll}f_{s} & f_{\mu_{0}} & f_{\mu_{2}}\end{array}\right]_{\left[(t), s^{*}, m(t), T(t), v(t), \mu_{0}^{*}, \mu_{2}^{*}\right.}^{T}\right]$

Grupo $\kappa=5$, conjuntos $\{2,2,1\},\{2,1,2\},\{1,2,2\}$

(i) Los mapas de observabilidad son

$\Phi_{\mathrm{I}}=\left[\begin{array}{lllll}\mathrm{m} & f_{\mathrm{m}} & \mathrm{T} & \mathrm{f}_{\mathrm{T}} & \mathrm{V}\end{array}\right]^{\mathrm{T}}, \Phi_{\mathrm{l}}=\left[\begin{array}{lllll}\mathrm{m} & \mathrm{f}_{\mathrm{m}} & \mathrm{T} & \mathrm{V} & \mathrm{f}_{\mathrm{V}}\end{array}\right]^{\mathrm{T}}, \Phi_{\mathrm{I}}=\left[\begin{array}{lllll}\mathrm{m} & \mathrm{T} & \mathrm{f}_{\mathrm{T}} & \mathrm{V} & \mathrm{f}_{\mathrm{V}}\end{array}\right]^{\mathrm{T}}$ (4.6a)

y se proponen los estados no observables

$\Phi_{\text {II }}=\left[\begin{array}{lll}s & \mu_{0} & \mu_{2}\end{array}\right]^{\mathrm{T}}$

con lo que los mapas de estimabilidad son

$$
\begin{aligned}
& \Phi=\left[\begin{array}{lllllll}
m & f_{m} & T & f_{T} & V & \mu_{0} & \mu_{2}
\end{array}\right]^{T} \\
& \Phi=\left[\begin{array}{lllllll}
m & f_{m} & T & V & f_{v} & \mu_{0} & \mu_{2}
\end{array}\right]^{T} \\
& \Phi=\left[\begin{array}{lllllll}
m & T & f_{T} & V & f_{v} & \mu_{0} & \mu_{2}
\end{array}\right]^{T}
\end{aligned}
$$

Con el conjunto $\{2,2,1\}$ se debe verificar que el mapa $\left[f_{m}, f_{T}\right]$ sea $I, s$-invertible. Del modelo del reactor se tiene:

$$
\begin{aligned}
& f_{m}=-r_{p o l}(I, m, s, T)+\left[\varepsilon_{m} r_{p o l}(I, m, s, T)+\varepsilon_{s} r_{s o l}(I, m, s, T)\right] m+\frac{q_{e}}{V}\left(m_{e}-m\right) \\
& f_{T}=\beta(m, s) r_{p}(I, m, s, T)-\left(T-T_{c}\right) \gamma(m, s, T, V)+\frac{q_{e}}{V}\left(\frac{1-\varepsilon_{m} m_{e}-\varepsilon_{s} s_{e}}{1-\varepsilon_{m} m-\varepsilon_{s} s}\right)(T-T)
\end{aligned}
$$

donde la tasa de polimerización total $r_{p o l}$ es:

$$
r_{p o l}=r_{p}+r_{m}+c_{1} r_{I}+c_{2} r_{\text {sol }} ; c_{1}=2 f_{I} / M^{0}, c_{2}=S^{0} / M^{\circ}
$$

El mapa $\left[f_{m}, f_{\mathrm{T}}\right]$ es $I_{,} s$-invertible si el siguiente determinante es diferente de cero:

$$
\operatorname{det}\left[\begin{array}{ll}
\frac{\partial f_{m}}{\partial I} & \frac{\partial f_{m}}{\partial s} \\
\frac{\partial f_{T}}{\partial I} & \frac{\partial f_{m}}{\partial s}
\end{array}\right] \neq 0
$$


La tasa de disociación del iniciador $\left(\mathrm{r}_{\mathrm{l}}\right)$ no depende del solvente; por otra parte, cuando se opera en régimen fuera del efecto gel existe poca dependencia de las tasas de propagación $\left(\mathrm{r}_{\mathrm{p}}\right)$ y transferencia a monómero $\left(r_{m}\right)$ con respecto al solvente, es decir:

$$
\frac{\partial \mathrm{r}_{\mathrm{i}}}{\partial \mathrm{s}}=0, \quad \frac{\partial \mathrm{r}_{\mathrm{p}}}{\partial \mathrm{s}} \approx 0, \quad \frac{\partial \mathrm{r}_{\mathrm{m}}}{\partial \mathrm{s}} \approx 0
$$

en tanto que la tasa de transferencia a solvente se reduce a:

$$
r_{s o l} \approx k_{f s}(T) s \lambda_{0}(I, T)=k_{f s}(T) s \sqrt{\lambda_{0 s}(I, T)}=k_{f s}(T) s \sqrt{\frac{2 f_{I} k_{d}(T) I}{k_{t 0}(T)}}
$$

y su dependencia con respecto al solvente depende de la magnitud de la constante $k_{f s}$ :

$$
\frac{\partial \mathrm{r}_{\mathrm{sol}}}{\partial \mathrm{s}} \approx \mathrm{k}_{\mathrm{fs}}(\mathrm{T}) \sqrt{\lambda_{0 \mathrm{~s}}}
$$

Expresando el determinante (4.6d) en términos cinéticos (tasas de reacción) y considerando la baja dependencia con respecto al solvente fuera del efecto gel se obtiene:

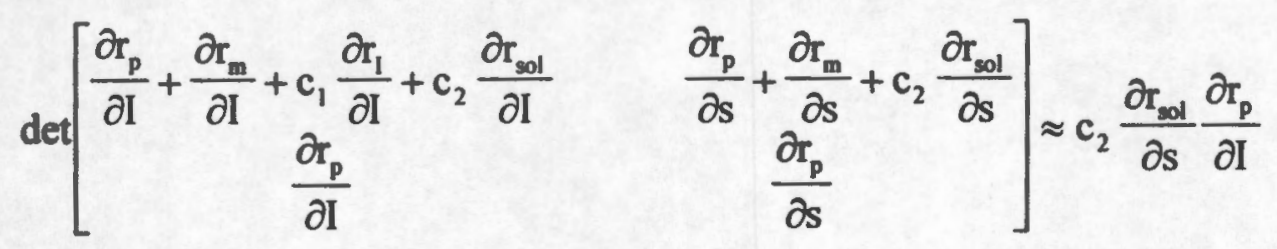

Debido a que la tasa de propagación aumenta del iniciador $\left(\partial \mathrm{r}_{\mathrm{p}} / \partial \mathrm{I}>0\right)$, la $I, s$-invertibilidad del mapa $\left[f_{m}, f_{\mathrm{r}}\right]$ dependerá de la constante de transferencia a solvente $(4.6 \mathrm{~g})$. Sin embargo, la reacción de transferencia a solvente es de varios órdenes de magnitud menor a la reacción de consumo de monómero por propagación (Odian, 1991), por lo que cuando se opera fuera del efecto gel, en general $f_{m}$ y $f_{T}$ poseen una débil dependencia de la concentración de solvente, haciendo que el solvente sea nominalmente (y por tanto robustamente) no observable y el mapa $\left[f_{m}, f_{\mathrm{T}}\right]$ no es $I, s$-invertible. El mapa $\Phi$ no es $x$-invertible y se descarta el conjunto $\{2,2,1\}$.

Los conjuntos de indices $\{2,1,2\},\{1,2,2\}$ requieren que los mapas [ $\left.f_{m}, f_{v}\right]$ y $\left[f_{T}, f_{v}\right]$ respectivamente sean $I, s$-invertibles. Del análisis con el conjunto $\{2,2,1\}$ se determinó cualitativamente que los térmicos cinéticos no presentan cambio apreciable con respecto a la concentración del solvente, debido a la baja dependencia fuera del efecto gel y a la baja tasa de transferencia. Los mismos términos cinéticos $\left(r_{l}, r_{p}, r_{s o l}, r_{p o l}\right)$ están involucrados en los mapas [ $f_{m}$ $\left.f_{\mathrm{V}}\right]$ y $\left[\mathrm{f}_{\mathrm{T}}, \mathrm{f}_{\mathrm{V}}\right]$, por lo que tales mapas tampoco son $I_{\mathrm{s}}$-invertibles y los correspondientes mapas $\Phi$ no son $x$-invertibles, descartándose los conjuntos $\{2,1,2\}$ y $\{1,2,2\}$. 
Grupo $\kappa=5$, conjuntos $\{3,1,1\},\{1,3,1\},\{1,1,3\}$

(i) Los mapas de observabilidad son

$$
\begin{aligned}
& \Phi_{1}=\left[\begin{array}{llllll}
m & f_{m} & L_{f} f_{m} & T & V
\end{array}\right]^{T}, \Phi_{1}=\left[\begin{array}{llllll}
m & T & f_{T} & L_{f} f_{T} & V
\end{array}\right]^{T} \\
& \Phi_{1}=\left[\begin{array}{llllll}
m & T & V & f_{V} & L_{f} f_{v}
\end{array}\right]^{T}
\end{aligned}
$$

No se continuará con el análisis de la x-invertibilidad de los mapas $\Phi$ debido a que estos conjuntos de índices serán descartados por las razones mencionadas en el siguiente inciso.

(ii) Los mapas $\varphi$ son L-continuos:

$$
\varphi=\left[\begin{array}{lll}
L_{f}^{2} f_{m} & f_{T} & f_{v}
\end{array}\right]^{T}, \varphi=\left[\begin{array}{lll}
f_{m} & L_{f}^{2} f_{T} & f_{v}
\end{array}\right]^{T}, \varphi=\left[\begin{array}{lll}
f_{m} & f_{T} & L_{f}^{2} f_{v}
\end{array}\right]^{T}
$$

Para estos conjuntos de índices de observabilidad aparecen en los mapas $\varphi$ segundas derivadas de la velocidad de polimerización y terceras derivadas de la concentración de monómero, temperatura y volumen; debido a que la aparición de derivadas de alto grado favorece una propagación de errores paramétricos y numéricos (afectando la robustez), estos conjuntos de índices se descartan; esta hipótesis se validará posteriormente. 
En resumen, del análisis de estimabilidad para las mediciones $y=[m, T, V]^{T}=\left[x_{3}, x_{4}, x_{5}\right]^{T}$ se obtienen los siguientes resultados cualitativos:

\begin{tabular}{|c|c|c|c|c|}
\hline $\begin{array}{c}\text { Grupo } \\
\kappa=\kappa_{\mathrm{m}}+\kappa_{\mathrm{T}}+\kappa_{\mathrm{V}}\end{array}$ & $\begin{array}{c}\text { Conjuntos } \\
\text { posibles } \\
k=\left\{\kappa_{\mathrm{m}}, \kappa_{\mathrm{T}}, \mathrm{K}_{\mathrm{v}}\right\}\end{array}$ & $\begin{array}{c}\begin{array}{c}\text { Dinámica } \\
\text { observable } \\
\left(x_{1}\right)\end{array} \\
\end{array}$ & $\begin{array}{c}\text { Estimación } \\
\text { nominal }\end{array}$ & $\begin{array}{c}\text { Estimación } \\
\text { robusta }\end{array}$ \\
\hline$\kappa=3$ & $\{1,1,1\}$ & $\mathrm{m}, \mathrm{T}, \mathrm{V}$ & Sí & Sí \\
\hline$\kappa=4$ & $\begin{array}{l}\{2,1,1\} \\
\{1,2,1\} \\
\{1,1,2\}\end{array}$ & $\mathbf{m}, \mathrm{T}, \mathrm{V}, \mathrm{I}$ & Sí & $\begin{array}{l}\text { Sí (1) } \\
\text { Sí (1) } \\
\text { No (2) }\end{array}$ \\
\hline$\kappa=5$ & $\begin{array}{l}\{3,1,1\} \\
\{1,3,1\} \\
\{1,1,3\} \\
\{2,2,1\} \\
\{2,1,2\} \\
\{1,2,2\}\end{array}$ & & \multicolumn{2}{|c|}{ No (3) } \\
\hline
\end{tabular}

Notas:

1.I-invertibilidad debido a que $r_{p}$ aumenta con el iniciador.

2.Se descarta $\{1,1,2\}$ debido a que es útil sólo a nivel laboratorio, en donde los cambios de volumen son apreciables.

3.Se descartan por la aparición de derivadas de alto grado, que pueden favorecer la propagación de errores numéricos y de modelado.

4.Se descartan ya que fuera del efecto gel la concentración de solvente es débilmente observable debido a la baja tasa de transferencia al mismo.

\section{Mediciones: densidad/índice de refracción, temperatura y volumen}

Se realizará el análisis de la resolubilidad cuando se utilizan densidad o índice de refracción en vez de concentración de monómero; el estudio con esta medición se empleará como referente. Las 
mediciones son:

$y=[\rho(m, s) / \eta(m, s), T, v]^{T}=\left[\rho\left(x_{3}, x_{2}\right) / \eta\left(x_{3}, x_{2}\right), x_{4}, x_{5}\right]^{T}$

donde $\rho(m, s)$ y $\eta(m, s)$ son la densidad y el índice de refracción, respectivamente, ambos dependientes de las concentraciones de monómero y solvente.

Del análisis realizado para las mediciones $\mathrm{y}=[\mathrm{m}, \mathrm{T}, \mathrm{V}]^{\mathrm{T}}=\left[\mathrm{x}_{3}, \mathrm{x}_{4}, \mathrm{x}_{5}\right]$, se concluyó que el entero $\kappa=\kappa_{m}+\kappa T+\kappa v$ puede ser 3 o 4. Cuando se tiene la densidad o al índice de refracción como medición (en vez de $m$ ) se obtiene el conjunto de ecuaciones mediante derivación sucesiva:

$$
\begin{aligned}
y_{1} & =\rho(m, s) / \eta \rho(m, s) \\
\dot{y}_{1} & =\dot{\rho} / \dot{\eta}=\frac{\partial(\rho / \eta)}{\partial m} \dot{m}+\frac{\partial(\rho / \eta)}{\partial s} \dot{s} \\
& =\frac{\partial(\rho / \eta)}{\partial m} f_{m}(I, s, m, T, V)+\frac{\partial(\rho / \eta)}{\partial s} f_{s}(I, s, m, T, V)
\end{aligned}
$$

las cuales no dependen de los momentos 0 y 2 . La conclusión de que el entero $\kappa$ puede ser $3 \circ 4$ puede extenderse al caso en que se considera la densidad como medición con las siguientes posibilidades:

\begin{tabular}{|c|c|}
\hline $\begin{array}{c}\text { Grupo } \\
\kappa=\kappa_{\mathrm{\rho} / \mathrm{n}}+\kappa_{\mathrm{T}}+\kappa_{\mathrm{V}}\end{array}$ & $\begin{array}{c}\text { Conjuntos posibles } \\
k=\left\{\kappa_{\mathrm{\rho} / \eta}, \kappa_{\mathrm{T}}, \kappa_{\mathrm{V}}\right\}\end{array}$ \\
\hline$\kappa=3$ & $\{1,1,1\}$ \\
\hline$\kappa=4$ & $\{2,1,1\},\{1,2,1\},\{1,1,2\}$ \\
\hline
\end{tabular}

donde no se consideran los conjuntos de índices para $\kappa=5$, en la hipótesis de que derivadas parciales de alto grado favorecen el comportamiento oscilatorio de los estimadores.

Grupo $x=3$, conjunto $\{1,1,1\}$

(i) Siendo el mapa de observabilidad $\Phi_{1}$ y el mapa $\Phi_{\text {II }}$ de estados no observables los siguientes:

$$
\begin{aligned}
& \Phi_{1}=\left[\begin{array}{lll}
\rho / \eta & T & V
\end{array}\right]^{T} \\
& \Phi_{11}=\left[\begin{array}{llll}
I & s & \mu_{0} & \mu_{2}
\end{array}\right]^{T}
\end{aligned}
$$

el mapa de estimabilidad $\Phi$ es 


$$
\Phi=\left[\begin{array}{lllllll}
\rho / \eta & T & V & I & s & \mu_{0} & \mu_{2}
\end{array}\right]^{\mathrm{T}}
$$

El mapa $\Phi$ es trivialmente $\mathrm{x}$-invertible (invertible para el vector de estados $x$ ) cuando se estudia con la concentración de monómero como medición; cuando se emplean densidad $(\rho)$ o índice de refracción $(\eta)$, los mapas $\Phi$ correspondientes son Rx-invertibles si ambas mediciones dependenden de la concentración de monómero, es decir si:

$\frac{\partial p}{\partial m} \neq 0, \frac{\partial \eta}{\partial m} \neq 0$

Para la densidad, a partir de un balance de masa (Apéndice D) la expresión es:

$$
\rho(m, s)=\frac{\rho_{m}^{\circ}}{1-\varepsilon_{m}}\left(1-\varepsilon_{m} m-\varepsilon_{s} s\right)
$$

y la derivada parcial con respecto al monómero es:

$$
\frac{\partial \rho}{\partial \mathrm{m}}=-\frac{\varepsilon_{\mathrm{m}} \rho_{\mathrm{m}}^{0}}{1-\varepsilon_{\mathrm{m}}}<0
$$

debido a que se cumple (4.9d), el mapa de estimabilidad con densidad es Rx-invertible. La derivada parcial de la densidad con respecto al monómero es igual a cero si la densidad del monómero puro es igual a la del polímero puro, es decir, si el factor de contracción $\left(\varepsilon_{\mathrm{m}}\right)$ es igual a cero.

Para el índice de refracción, existe una correlación en función de monómero y solvente por Lorimer, (1972) (detalles en el apéndice C):

$$
\eta(m, s)=\eta_{0}(m, s)+v c_{p o l}(m, s)+a_{2}(m, s) c_{p o l}^{2}(m, s)
$$

En general el índice de refracción disminuye con la concentración de monómero; a partir de la correlación de Lorimer se determina que (demostrado en apéndice C):

$$
\frac{\partial \eta}{\partial \mathrm{m}}<0
$$

debido a que se cumple (4.9d), el mapa de estimabilidad con índice de refracción es Rx-invertible.

(ii) El mapa $\varphi$ es L-continuo:

$\varphi=\left[\frac{\partial(\rho / \eta)}{\partial m} f_{m}+\frac{\partial(\rho / \eta)}{\partial s} f_{s} \quad f_{T} \quad f_{v}\right]^{T}$ 
(iii) La dinámica no observable es RE-estable:

$\left.\left[\begin{array}{llll}\dot{I}^{*} & \dot{s}^{*} & \ddot{\mu}_{0}^{*} & \ddot{\mu}_{2}^{*}\end{array}\right]^{T}=\left[\begin{array}{llll}f_{I} & f_{s} & f_{\mu_{0}} & f_{\mu_{2}}\end{array}\right]_{\left[I^{*}, s^{*}, p(t) / \eta(t), T(t), V(t), \mu_{0}^{*}, \mu_{2}^{*}\right.}^{T}\right]$

Grupo $\kappa=4$, conjuntos $\{2,1,1\},\{1,2,1\},\{1,1,2\}$

(i) Los mapas de observabilidad respectivos son

$$
\begin{aligned}
& \Phi_{1}=\left[\begin{array}{llll}
\rho / \eta & \dot{\rho} / \dot{\eta} & T & V
\end{array}\right]^{T}=\left[\begin{array}{llll}
\rho / \eta & \frac{\partial(\rho / \eta)}{\partial m} f_{m}+\frac{\partial(\rho / \eta)}{\partial s} f_{s} & T & V
\end{array}\right]^{T} \\
& \Phi_{1}=\left[\begin{array}{llll}
\rho / \eta & T & f_{T} & V
\end{array}\right]^{T} \\
& \Phi_{1}=\left[\begin{array}{llll}
\rho / \eta & T & V & f_{V}
\end{array}\right]^{T}
\end{aligned}
$$

se proponen los estados no observables

$$
\Phi_{I I}=\left[\begin{array}{lll}
s & \mu_{0} & \mu_{2}
\end{array}\right]^{\mathrm{T}}
$$

con lo que los mapas de estimabilidad son

$$
\begin{aligned}
& \Phi=\left[\begin{array}{llllllll}
\rho / \eta & \frac{\partial(\rho / \eta)}{\partial m} f_{m} & +\frac{\partial(\rho / \eta)}{\partial s} f_{s} & T & V & s & \mu_{0} & \mu_{2}
\end{array}\right]^{T} \\
& \Phi=\left[\begin{array}{llllllll}
m / \rho / \eta & T & f_{T} & V & s & \mu_{0} & \mu_{2}
\end{array}\right]^{T} \\
& \Phi=\left[\begin{array}{lllllll}
m / \rho / \eta & T & V & f_{V} & s & \mu_{0} & \mu_{2}
\end{array}\right]^{T}
\end{aligned}
$$

Como en general tanto la densidad como el índice de refracción disminuyen con la concentración de monómero $(\partial \rho / \partial \mathrm{m}<0, \partial \eta / \partial \mathrm{m}<0)$ por lo que ambas mediciones son invertibles para el monómero. Para determinar la invertibilidad del iniciador se parte de las propiedades físicas consideradas anteriormente $\left(\partial \mathrm{r}_{\mathrm{pol}} / \partial \mathrm{I}>0, \partial \mathrm{r}_{\mathrm{sol}} / \partial \mathrm{I}>0\right.$ y $\left.\partial \mathrm{r}_{\mathrm{pol}} / \partial \mathrm{I}>\partial \mathrm{r}_{\mathrm{sol}} / \partial \mathrm{I}\right)$ y considerando únicamente la cinética del monómero se obtiene para el conjunto $\{2,1,1\}$ :

$$
\dot{\rho} / \dot{\eta}=\frac{\partial(\rho / \eta)}{\partial m} f_{m}+\frac{\partial(\rho / \eta)}{\partial s} f_{s} \approx \frac{\partial(\rho / \eta)}{\partial m} f_{m}
$$

dado que el mapa $f_{m}$ es $I$-invertible nominal (4.5d) y robustamente (4.5g):

$$
\frac{\partial f_{m}}{\partial I}<0
$$


entonces (4.10d) es invertible para el iniciador, por lo que el mapa de estimabilidad correspondiente es $\mathrm{x}$-invertible.

Para los otros conjuntos de índices de observabilidad $(\{1,2,1\}$ y $\{1,1,2\})$, ya se analizó que nominalmente los mapas $\left\{\mathrm{f}_{\mathrm{T}}, \mathrm{f}_{\mathrm{v}}\right\}$ dependen del iniciador $(4.5 \mathrm{e}, \mathrm{f})$ :

$\frac{\partial \mathrm{f}_{\mathrm{T}}}{\partial \mathrm{I}}>0 \quad \frac{\partial \mathrm{f}_{\mathrm{v}}}{\partial \mathrm{I}}<0$

por lo que cada mapa del conjunto $\left\{\mathrm{f}_{\mathrm{T}}, \mathrm{f}_{\mathrm{V}}\right\}$ es $I$-invertible, y los mapas $\Phi$ correspondientes son $\mathrm{x}$ invertibles. Para la determinación de la estimabilidad robusta, a partir de (4.5i) se observa que el mapa $f_{V}$ no es $\mathrm{R} I$-invertible, por lo que se descarta el conjunto $\{1,1,2\}$.

(ii) Los mapas $\varphi$ (para $\{2,1,1\}$ y $\{1,2,1\})$ son L-continuos:

$$
\begin{aligned}
& \varphi=\left[L_{f}\left(\frac{\partial(\rho / \eta)}{\partial m} f_{m}\right)+L_{f}\left(\frac{\partial(\rho / \eta)}{\partial s} f_{s}\right) \quad f_{T} \quad f_{v}\right]^{T} \\
& \varphi=\left[\frac{\partial(\rho / \eta)}{\partial m} f_{m}+\frac{\partial(\rho / \eta)}{\partial s} f_{s} \quad L_{f} f_{T} \quad f_{v}\right]^{T}
\end{aligned}
$$

(iii) La dinámica no observable es RE-estable:

$$
\left.\left[\begin{array}{lll}
\dot{s} & \ddot{\mu}_{0}^{*} & \ddot{\mu}_{2}^{*}
\end{array}\right]^{T}=\left[\begin{array}{lll}
f_{3} & f_{\mu_{0}} & f_{\mu_{2}}
\end{array}\right]_{\left[(t), s ; 0(t) / \eta(t), T(t), v(t), \mu_{0}^{*}, \mu_{2}^{*}\right.}^{T}\right]
$$

En resumen, del análisis de estimabilidad para las mediciones $y=[\rho(m, s) / \eta(m, s), T, v]^{T}=\left[\rho\left(x_{3}, x_{2}\right) / \eta\left(x_{3}, x_{2}\right), x_{4}, x_{5}\right]^{T}$ se obtienen los siguientes resultados cualitativos:

\begin{tabular}{|c|c|c|c|c|}
\hline $\begin{array}{c}\text { Grupo } \\
\kappa=\kappa_{\mathrm{p} / \mathrm{n}}+\kappa_{\mathrm{T}}+\kappa_{\mathrm{v}}\end{array}$ & $\begin{array}{c}\text { Conjuntos } \\
\text { posibles } \\
k=\left\{\kappa_{\mathrm{o} / \mathrm{n}}, \kappa_{\mathrm{T}}, \kappa_{\mathrm{v}}\right\}\end{array}$ & $\begin{array}{c}\text { Dinámica } \\
\text { observable } \\
\left(\mathrm{x}_{1}\right)\end{array}$ & $\begin{array}{c}\text { Estimación } \\
\text { nominal }\end{array}$ & $\begin{array}{c}\text { Estimación } \\
\text { robusta }\end{array}$ \\
\hline$\kappa=3$ & $\{1,1,1\}$ & $\mathrm{m}, \mathrm{T}, \mathrm{V}$ & $\mathrm{Si}$ & $\mathrm{Si}(1)$ \\
\hline$\kappa=4$ & $\begin{array}{l}\{2,1,1\} \\
\{1,2,1\}\end{array}$ & $\mathrm{m}, \mathrm{T}, \mathrm{V}, \mathrm{I}$ & $\mathrm{Si}$ & $\begin{array}{c}\text { Si }(1,2) \\
\mathrm{Si}(1,2) \\
\mathrm{No}(3)\end{array}$ \\
\hline
\end{tabular}




\section{Notas:}

1. $m$-invertibilidad debido a que $\rho$ o $\eta$ disminuyen con el monómero.

2.I-invertibilidad debido a que $r_{p}$ aumenta con el iniciador.

3.Se descarta $\{1,1,2\}$ debido a que es útil sólo a nivel laboratorio, en donde los cambios de volumen son apreciables.

\section{Mediciones: indice de refracción, densidad, temperatura y volumen}

En el anterior estudio de resolubilidad se consideró la posibilidad de estudiar densidad o índice de refracción; en el estudio siguiente se emplearán ambas mediciones simultáneamente:

$y=[\eta(m, s), \rho(m, s), T, V]^{T}=\left[\eta\left(x_{3}, x_{2}\right), \rho\left(x_{3}, x_{2}\right), x_{4}, x_{5}\right]^{T}$

Del análisis realizado para las mediciones $y=[m, T, V]^{T}=\left[x_{3}, x_{4}, x_{5}\right]$, se concluyó que el entero $\kappa=\kappa_{m}+\kappa_{T}+\kappa_{V}$ puede ser 304 , conclusión que se extendió al caso de cuando se considera la densidad o el índice de refracción como medición. En general la concentración de solvente es no observable; la inclusión del solvente en la dinámica observable verifica con una medición adicional dependiente de este estado. Considerando como mediciones la densidad, el índice de refracción, la temperatura y el volumen, los conjuntos posibles de indices de observabilidad son los siguientes:

\begin{tabular}{|c|c|}
\hline $\begin{array}{c}\text { Grupo } \\
\kappa=\kappa_{\mathrm{n}}+\kappa_{\mathrm{0}}+\kappa_{\mathrm{T}}+\kappa_{\mathrm{V}}\end{array}$ & $\begin{array}{l}\text { Conjuntos posibles } \\
k=\left\{\kappa_{\mathrm{n}}, \kappa_{0}, \kappa_{\mathrm{T}}, \kappa_{\mathrm{V}}\right\}\end{array}$ \\
\hline$\kappa=4$ & $\{1,1,1,1\}$ \\
\hline$\kappa=5$ & $\{2,1,1,1\},\{1,2,1,1\},\{1,1,2,1\},\{1,1,1,2\}$ \\
\hline
\end{tabular}

Grupo $\kappa=4$, conjunto $\{1,1,1,1\}$

(i) Siendo el mapa de observabilidad $\Phi_{1}$ y el mapa $\Phi_{I I}$ de estados no observables los siguientes:

$\Phi_{1}=\left[\begin{array}{llll}\eta & \rho & T & V\end{array}\right]^{\top}$ 


$$
\Phi_{\mathrm{II}}=\left[\begin{array}{lll}
\mathrm{I} & \mu_{0} & \mu_{2}
\end{array}\right]^{\mathrm{T}}
$$

el mapa de estimabilidad $\Phi$ es

$$
\Phi=\left[\begin{array}{lllllll}
\eta & \rho & T & V & I & \mu_{0} & \mu_{2}
\end{array}\right]^{T}
$$

El mapa de estimabilidad $\Phi$ es Rx-invertible si a partir de la densidad e índice de refracción es posible conocer simultáneamente la concentración de monómero y la de solvente, es decir, si $\eta$ y $\rho$ son $m, s$-resolubles. El mapa $[\rho, \eta]$ es $m, s$-invertible si el siguiente determinante es diferente de cero:

$$
\operatorname{det}\left[\begin{array}{ll}
\frac{\partial \rho}{\partial m} & \frac{\partial \rho}{\partial s} \\
\frac{\partial \eta}{\partial m} & \frac{\partial \eta}{\partial s}
\end{array}\right]=\frac{\partial \rho}{\partial m} \frac{\partial \eta}{\partial s}-\frac{\partial \rho}{\partial s} \frac{\partial \eta}{\partial m} \neq 0
$$

En general el determinante (4.12d) es diferente de cero, por lo que las mediciones $\rho$ y $\eta$ son $m, s-$ resolubles. La condición para que tales mediciones no sean $m, s$-resolubles (determinante igual a cero) es la de que los índices de refracción del monómero y solvente puros deben ser iguales y lo mismo con las densidades del monómero y solvente puros, es decir:

$$
\operatorname{det}\left[\frac{\partial(\rho, \eta)}{\partial(m, s)}\right]=0 \leftrightarrow \rho_{m}^{\circ}=\rho_{s}^{\circ}, \eta_{m}=\eta_{s}
$$

Debido a la resolubilidad para $m$ y $s$ a partir de $\rho$ y $\eta$, el mapa de estimabilidad es Rx-invertible.

(ii) El mapa $\varphi$ es L-continuo:

$$
\varphi=\left[\begin{array}{lllll}
\frac{\partial \rho}{\partial m} f_{m}+\frac{\partial \rho}{\partial s} f_{s} & \frac{\partial \eta}{\partial m} f_{m}+\frac{\partial \eta}{\partial s} f_{s} & f_{T} & f_{v}
\end{array}\right]^{T}
$$

(iii) La dinámica no observable es RE-estable:

$$
\left.\left[\begin{array}{lll}
\dot{I}^{*} & \ddot{\mu}_{0}^{*} & \ddot{\mu}_{2}^{*}
\end{array}\right]^{T}=\left[\begin{array}{lll}
f_{I} & f_{\mu_{0}} & f_{\mu_{2}}
\end{array}\right]_{\left[r^{*}, \eta(t), \rho(t), T(t), V(t), \mu_{0}^{*}, \mu_{2}^{*}\right.}^{T}\right]
$$

Grupo $\kappa=5$, conjuntos $\{2,1,1,1\},\{1,2,1,1\},\{1,1,2,1\},\{1,1,1,2\}$

(i) Los mapas de observabilidad respectivos son

$$
\Phi_{\mathrm{I}}=\left[\begin{array}{lllll}
\eta & \dot{\eta} & \rho & \mathrm{T} & \mathrm{V}
\end{array}\right]^{\mathrm{T}}=\left[\begin{array}{lllll}
\eta & \frac{\partial \eta}{\partial \mathrm{m}} \mathrm{f}_{\mathrm{m}}+\frac{\partial \eta}{\partial \mathrm{s}} \mathrm{f}_{\mathrm{s}} & \rho & \mathrm{T} & \mathrm{V}
\end{array}\right]^{\mathrm{T}}
$$




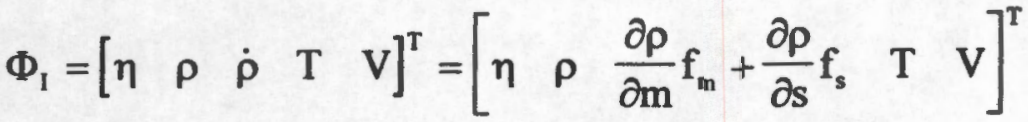

$$
\begin{aligned}
& \Phi_{1}=\left[\begin{array}{lllll}
\eta & \rho & \mathrm{T} & \mathrm{f}_{\mathrm{T}} & \mathrm{V}
\end{array}\right]^{\mathrm{T}}, \Phi_{\mathrm{I}}=\left[\begin{array}{lllll}
\eta & \rho & \mathrm{T} & \mathrm{V} & \mathrm{f}_{\mathrm{V}}
\end{array}\right]^{\mathrm{T}}
\end{aligned}
$$

se proponen los estados no observables

$$
\Phi_{\mathrm{n}}=\left[\begin{array}{ll}
\mu_{0} & \mu_{2}
\end{array}\right]^{\mathrm{T}}
$$

con lo que los mapas de estimabilidad son

$$
\begin{aligned}
& \Phi=\left[\begin{array}{llllllll}
\eta & \frac{\partial \eta}{\partial m} f_{m}+\frac{\partial \eta}{\partial s} f_{s} & \rho & T & V & \mu_{0} & \mu_{2}
\end{array}\right]^{T} \\
& \Phi=\left[\begin{array}{llllllll}
\eta & \rho & \frac{\partial \rho}{\partial m} f_{m}+\frac{\partial \rho}{\partial s} f_{s} & T & V & \mu_{0} & \mu_{2}
\end{array}\right]^{T} \\
& \Phi=\left[\begin{array}{llllllll}
\eta & \rho & T & f_{T} & V & \mu_{0} & \mu_{2}
\end{array}\right]^{T} \\
& \Phi=\left[\begin{array}{lllllllll}
\eta & \rho & T & V & f_{v} & \mu_{0} & \mu_{2}
\end{array}\right]^{T}
\end{aligned}
$$

Como en general se cumple (4.12d), índice de refracción y densidad son invertibles para monómero y solvente. Para determinar la invertibilidad del iniciador se parte de las propiedades fisicas consideradas anteriormente $\left(\partial \mathrm{r}_{\mathrm{pol}} / \partial \mathrm{I}>0, \partial \mathrm{r}_{\text {sol }} / \partial \mathrm{I}>0\right.$ y $\left.\partial \mathrm{r}_{\mathrm{pol}} / \partial \mathrm{I}>\partial \mathrm{r}_{\mathrm{sol}} / \partial \mathrm{I}\right)$ y considerando únicamente la cinética del monómero se obtiene:

$$
\dot{\eta}=\frac{\partial \eta}{\partial m} f_{m}+\frac{\partial \eta}{\partial s} f_{s} \approx \frac{\partial \eta}{\partial m} f_{m} \text { para }\{2,1,1,1\}
$$

o bien

$\dot{\rho}=\frac{\partial \rho}{\partial m} f_{m}+\frac{\partial \rho}{\partial s} f_{s} \approx \frac{\partial \rho}{\partial m} f_{m}$ para $\{1,2,1,1\}$

dado que el mapa $f_{m}$ es $I$-invertible nominal $(4.5 \mathrm{~d})$ y robustamente $(4.5 \mathrm{~g})$ :

$\frac{\partial \mathrm{f}_{\mathrm{m}}}{\partial \mathrm{I}}<0$

entonces $(4.13 \mathrm{~d}, \mathrm{e})$ son resolubles para el iniciador, por lo que los mapas de estimabilidad correspondientes son $\mathbf{x}$-invertibles.

Para los otros conjuntos de índices de observabilidad $(\{1,1,2,1\}$ y $\{1,1,1,2\})$, ya se analizó que nominalmente los mapas $\left\{f_{\mathrm{T}}, \mathrm{f}_{\mathrm{v}}\right\}$ dependen del iniciador $(4.5 \mathrm{e}, \mathrm{f})$ : 
$\frac{\partial \mathrm{f}_{\mathrm{T}}}{\partial \mathrm{I}}>0 \quad \frac{\partial \mathrm{f}_{\mathrm{V}}}{\partial \mathrm{I}}<0$

por lo que cada mapa del conjunto $\left\{f_{\mathrm{T}}, f_{\mathrm{V}}\right\}$ es $I$-invertible, y los mapas $\Phi$ correspondientes son $\mathrm{x}$ invertibles. Para la determinación de la estimabilidad robusta, a partir de (4.5i) se observa que el mapa $f_{V}$ no es $\mathrm{R} I$-invertible, por lo que se descarta el conjunto $\{1,1,1,2\}$.

(ii) Los mapas $\varphi$ (para $\{2,1,1,1\},\{1,2,1,1\}$ y $\{1,1,2,1\})$ son L-continuos:

$$
\begin{aligned}
& \varphi=\left[L_{f}\left(\frac{\partial \eta}{\partial m} f_{m}\right)+L_{f}\left(\frac{\partial \eta}{\partial s} f_{s}\right) \frac{\partial \rho}{\partial m} f_{m}+\frac{\partial \rho}{\partial s} f_{s} \quad f_{T} \quad f_{V}\right]^{T} \\
& \varphi=\left[\begin{array}{llll}
\frac{\partial \eta}{\partial m} f_{m}+\frac{\partial \eta}{\partial s} f_{s} & \frac{\partial \rho}{\partial m} L_{f} f_{m}+L_{f} \frac{\partial \rho}{\partial s} f_{s} & f_{T} & f_{V}
\end{array}\right]^{T} \\
& \varphi=\left[\begin{array}{llll}
\frac{\partial \eta}{\partial m} f_{m}+\frac{\partial \eta}{\partial s} f_{s} & \frac{\partial \rho}{\partial m} f_{m}+\frac{\partial \rho}{\partial s} f_{s} & L_{f} f_{T} & f_{v}
\end{array}\right]^{T}
\end{aligned}
$$

(iii) La dinámica no observable es RE-estable:

$\left.\left[\begin{array}{ll}\ddot{\mu}_{0}^{*} & \ddot{\mu}_{2}^{*}\end{array}\right]^{T}=\left[\begin{array}{ll}f_{\mu_{0}} & f_{\mu_{2}}\end{array}\right]_{\left[I(t), \eta(t), P(t), T(t), V(t), \mu_{0}, \mu_{2}^{*}\right.}^{T}\right]$

En resumen, del análisis de estimabilidad para las mediciones $y=[\eta(m, s), \rho(m, s), T, v]^{T}=\left[\eta\left(x_{3}, x_{2}\right), \rho\left(x_{3}, x_{2}\right), x_{4}, x_{3}\right]^{T}$ se obtienen los siguientes resultados cualitativos:

\begin{tabular}{|c|c|c|c|c|}
\hline $\begin{array}{c}\text { Grupo } \\
\kappa=\kappa_{\eta}+\kappa_{\mathrm{p}}+\kappa_{\mathrm{T}}+\kappa_{\mathrm{V}}\end{array}$ & $\begin{array}{c}\text { Conjuntos posibles } \\
k=\left\{\kappa_{\eta}, \kappa_{\mathrm{p}}, \kappa_{\mathrm{T}}, \kappa_{\mathrm{V}}\right\}\end{array}$ & $\begin{array}{c}\text { Dinámica } \\
\text { observable } \\
\left(\mathrm{x}_{1}\right)\end{array}$ & $\begin{array}{c}\text { Estimación } \\
\text { nominal }\end{array}$ & $\begin{array}{c}\text { Estimación } \\
\text { robusta }\end{array}$ \\
\hline$\kappa=4$ & $\{1,1,1,1\}$ & $\mathrm{m}, \mathrm{s}, \mathrm{T}, \mathrm{V}$ & Sí & Sí (1) \\
\hline$\kappa=5$ & $\left\{\begin{array}{l}\{1,1,1,1\} \\
\{1,2,1,1\} \\
\{1,1,2,1\}\end{array}\right.$ & $\mathrm{m}, \mathrm{s}, \mathrm{T}, \mathrm{V}, \mathrm{I}$ & Sí & $\begin{array}{c}\text { Si }(1,2) \\
\text { Sí }(1,2) \\
\text { Si }(1,2) \\
\text { No (3) }\end{array}$ \\
\hline
\end{tabular}


Notas:

1. $m, s$-invertibilidad siempre que $\rho_{m}^{0} \neq \rho_{s}^{0}$ y/o $\eta_{m} \neq \eta_{s}$

2.1-invertibilidad debido a que $r_{p}$ aumenta con el iniciador.

3.Se descarta $\{1,1,1,2\}$ debido a que es útil sólo a nivel laboratorio, en donde los cambios de volumen son apreciables.

La resolubilidad del problema de estimación es una consecuencia de tres propiedades físicas que se presentan en cualquier reactor de homopolimerización por radicales libres (en masa o solución):

(1) La densidad o el índice de refracción disminuyen con la concentración de monómero.

(2) La velocidad de polimerización se incrementa con la concentración de iniciador.

(3) La dinámica del monómero es la única fuente de inestabilidad en el reactor debido al efecto gel.

Por otra parte, a nivel industrial no resulta útil un índice de observabilidad alto para la medición de volumen, debido a la baja sensibilidad de los medidores industriales de nivel.

La concentración de solvente se incluye en los estados de la dinámica observable si se emplean simultáneamente mediciones de densidad e índice de refracción. En caso de que se incluyan ambas mediciones, la resolubilidad depende asimismo de la diferencia entre las propiedades de los compuestos puros.

Se verificó cualitativamente de que en caso de que sólo se disponga de una de las mediciones anteriores, el solvente puede formar parte de la dinámica observable si el reactor opera en régimen de efecto gel o si la reacción de transferencia a solvente es significativa.

La relevancia del análisis realizado en este capítulo radica en que proporciona una orientación sobre las estructuras de estimabilidad (conjuntos de índices de observabilidad y estados de la dinámica observable) para las cuales es resoluble el problema. Con base en el análisis se construyen y sintonizan los estimadores (apéndice E) y se verificarán los desempeños en el siguiente capítulo. 


\section{Capítulo V. Casos de estudio}

En este capítulo se presentarán los resultados que siguen al análisis de resolubilidad del problema de estimación. Se estudia el desempeño de estimadores no lineales para dos casos de estudio con datos experimentales reportados en literatura. Para cada conjunto de mediciones de los casos experimentales se utilizaron diferentes índices de observabilidad.

\section{Condiciones de operación}

Para corroborar los resultados obtenidos en el capítulo anterior y comparar el desempeño de los estimadores construidos a partir de diferentes conjuntos de observabilidad; se emplearon los modelos y datos experimentales de dos casos reportados en la literatura:

a) Polimerización de metilmetacrilato (MMA) con etil-acetato (EA) como solvente y azobisisobutironitrilo (AIBN) como iniciador, en un reactor en lote.

b) Polimerización de MMA con tolueno (TOL) como solvente y AIBN como iniciador, en un reactor continuo con excitación periódica de temperatura y flujo de entrada de iniciador.

Las condiciones de operación utilizadas para el reactor en lote son descritas por Ellis y col. (1994) y se encuentran resumidas en la Tabla 5.1. Se realizaron tres corridas a diferentes temperaturas. En las estimaciones se empleó la temperatura de $60^{\circ} \mathrm{C}$, debido a que a tal temperatura el modelo describe mejor los datos experimentales. Se utilizarán como mediciones densidad y volumen (en un reactor en lote, el volumen resulta conocido directamente a través de la densidad y la masa inicial total).

Las condiciones para el reactor continuo son descritas por Mutha y col. (1997) y se resumen en la Tabla 5.2. El reactor opera con cambios en escalón en la temperatura y flujo de iniciador; la secuencia de cambios se mostrará posteriormente. Las mediciones a emplear serán densidad y temperatura.

Los valores utilizados en las constantes de reacción y constantes fisicas son los reportados por Alvarez y col. (1990), a excepción de las constantes de transferencia a monómero y transferencia a solvente, cuyos valores fueron tomados de Ellis y col. (1994) y de Mutha y col. (1997). 
Tabla 5.1. Condiciones de operación en un reactor en lote (Ellis y col., 1994)

\begin{tabular}{|c|c|}
\hline Temperatura $\left({ }^{\circ} \mathrm{C}\right)$ & $50,60,70$ \\
\hline Monómero $(\%$ peso, MMA) & 25 \\
\hline Volumen de solvente $(\mathrm{ml}, \mathrm{EA})$ & 2,842 \\
\hline Concentración de iniciador $(\mathrm{M}, \mathrm{AIBN})$ & 0.05 \\
\hline
\end{tabular}

Tabla 5.2. Condiciones de operación en un reactor continuo (Mutha y col., 1997)

\begin{tabular}{|c|c|}
\hline Temperatura $\left({ }^{\circ} \mathrm{C}\right)$ & $70-90$ \\
\hline Tasa de alimentación de monómero (L/min, MMA) & 0.0133 \\
\hline Tasa de alimentación de solvente (L/min, TOL) & 0.02 \\
\hline Volumen de reactor $(\mathrm{L})$ & 1.3 \\
\hline Concentración de iniciador en la alimentación (M, AIBN) & 0.032 \\
\hline Tiempo de residencia (min) & 40 \\
\hline
\end{tabular}

El problema de estimación consiste en la obtención de estimados de la conversión $(c)$, la fracción masa de sólidos $(\phi)$, el peso molecular en número $\left(M_{n}\right)$ y el peso molecular en peso $\left(M_{w}\right)$. Los trabajos experimentales estudiados no muestran la fracción de sólidos experimental, por lo que mostrarán las predicciones de los estimadores. Se presentarán los resultados divididos en dos partes: (a) Resultados para un reactor en lote (con las condiciones de Ellis y col.), y (b) resultados para un reactor continuo (con las condiciones de Mutha y col.).

\section{Mapas de salida}

Por consistencia en el modelado, además del empleo de los parámetros de Ellis y col., y Mutha y col., se utilizaron los mapas de salida reportados en tales trabajos (conversión y densidad). En el análisis con el reactor de Ellis y col. se empleará para la densidad y conversión estimadas las siguientes expresiones (deducción en el apéndice D):

$$
\begin{aligned}
& \rho(m, V)=\frac{\rho_{m}^{\circ}}{1-\varepsilon_{m}}\left(1-\varepsilon_{m} m-\varepsilon_{s} \frac{V_{s}}{V}-\varepsilon_{1} \frac{V_{1}}{V}\right) \\
& c=\frac{\rho-\frac{W_{T}}{\rho_{m}^{\circ}}-\frac{W_{s}}{\rho_{s}^{0}}-\frac{W_{1}}{\rho_{1}^{0}}}{\frac{w_{m}}{\rho_{\mathrm{p}}^{0}}-\frac{w_{m}}{\rho_{m}^{0}}}
\end{aligned}
$$

donde $\varepsilon_{m}, \varepsilon_{s}, \varepsilon_{l}$ son factores de contracción por la diferencia de densidad entre el polímero, y el 
monómero, solvente e iniciador, respectivamente; $V_{s}$ y $V_{I}$ son los volúmenes de solvente e iniciador agregados inicialmente al reactor; $w_{T}$ es la carga inicial total; $w_{m}, w_{s}, w_{I}$ son las cargas iniciales de monómero, solvente, iniciador. La expresión de la densidad (5.5a) se obtuvo mediante aditividad de volúmenes, en tanto que la expresión de conversión es reportada por Ellis y col. Para el reactor de Mutha y col. las expresiones a utilizar son:

$$
\begin{aligned}
& \rho(m, s)=\frac{(1-s)\left(1-\varepsilon_{m}\right)}{(1-s)-\varepsilon_{m}(2-2 s-m)} \rho_{c} \\
& c=\frac{1-\varepsilon_{m}}{\varepsilon_{m}}\left(1-\frac{\rho_{\mathrm{c}}}{\rho}\right)
\end{aligned}
$$

$\rho_{e}$ es la densidad del flujo de entrada (monómero y solvente). Las deducciones de (5.6a) y (5.6b) se encuentran en el apéndice $D$.

\section{Conjuntos de índices de observabilidad}

De acuerdo con el análisis de estimabilidad realizado en el capítulo anterior, debido a que el reactor tiene 7 estados y 3 mediciones (densidad, temperatura y volumen), existen matemáticamente 35 conjuntos posibles de índices de observabilidad; tal número se puede reducir tomando en cuenta ciertas consideraciones relacionadas con el modelo y aspectos de ingeniería:

a) El mapa $h$ y los primeros 5 mapas $\left(f_{1}, \ldots, f_{5}\right)$ no dependen de los momentos $\left(x_{6}\right.$ y $\left.x_{7}\right)$, implicando que estos estados se encuentran en la dinámica no observable, por lo que el mapa de estimabilidad $\Phi_{1}$ es de dimensión menor o igual a 5. Es decir, el entero $\kappa=\kappa_{\rho}+\kappa_{T}+\kappa v$ puede ser 3,4 o 5. Por otra parte, los mapas $\left(f_{1}, \ldots, f_{5}\right)$ dependen débilmente del solvente debido a la baja tasa de transferencia al mismo; la concentración de solvente se encuentra fundamentalmente en la dinámica no observable (en la dinámica observable no hay información significativa de $s$ ) y el entero $\kappa$ puede ser 3 o 4.

b) En un reactor industrial, los cambios en volumen debidos a la conversión no se pueden detectar satisfactoriamente por un medidor de nivel o gravimétrico (Alvarez y Lopez, 1999), por lo que el índice de observabilidad de volumen $k v$ debe ser igual a 1.

c) Para evitar una propagación excesiva de errores numéricos y de modelado, el mapa $\varphi$ contendrá hasta la primera derivada de la velocidad de polimerización y segunda derivada de la 
densidad ( $\left.\kappa_{p}, \kappa_{T} \leq 2\right)$, entendiéndose que la hipótesis de propagación de errores se validará posteriormente.

A partir de estas consideraciones, el número de conjuntos de índices se reduce a 3, clasificados en dos grupos de acuerdo al entero $\kappa$.

Tabla 5.3. Conjuntos de indices de observabilidad y vectores de estados observables y no observables

\begin{tabular}{|c|c|c|c|}
\hline Entero $\mathrm{K}$ & Conjuntos de indices $\mathrm{C}_{\mathbf{i}}$ & Estados observables $\mathbf{x}_{\mathbf{I}}$ & Estados no observables $\mathbf{x}_{\mathbf{n}}$ \\
\hline 3 & $\mathrm{C}_{1}=\{1,1,1\}$ & $(\mathrm{m}, \mathrm{T}, \mathrm{V})$ & $\left(\mathrm{I}, \mathrm{s}, \mu_{0}, \mu_{2}\right)$ \\
\hline 4 & $\mathrm{C}_{2}=\{2,1,1\}$ & $(\mathrm{m}, \mathrm{T}, \mathrm{V}, \mathrm{I})$ & $\left(\mathrm{s}, \mu_{0}, \mu_{2}\right)$ \\
\hline
\end{tabular}

\section{Estimabilidad robusta}

\section{Invertibilidad de los mapas $\Phi$}

Para $C_{1}$ la inclusión de la concentración de monómero en la dinámica observable se basa en el principio fisico de que la densidad disminuye con el aumento en la concentración de monómero, es decir:

$$
\frac{\partial p}{\partial m}<0
$$

lo que se cumple para el mapa de salida (4.9):

$$
\frac{\partial \rho}{\partial m}=-\frac{\varepsilon_{m} \rho_{m}^{\circ}}{1-\varepsilon_{m}}<0
$$

así como para los mapas de salida de Ellis y col., y Mutha y col.:

$$
\begin{array}{ll}
\frac{\partial \rho}{\partial m}=-\frac{\varepsilon_{\mathrm{m}} \rho_{\mathrm{m}}^{\circ}}{1-\varepsilon_{\mathrm{m}}}<0 & \text { (Ellis y col.) } \\
\frac{\partial \rho}{\partial \mathrm{m}}=-\frac{\varepsilon_{\mathrm{m}}(1-s)\left(1-\varepsilon_{\mathrm{m}}\right)}{(1-s)-\varepsilon_{\mathrm{m}}(2-2 s-\mathrm{m})} \rho_{\mathrm{e}}<0 & \text { (Mutha y col.) }
\end{array}
$$

con lo que el mapa $\Phi$ para $C_{1}$ es $x$ - invertible. Por otra parte, para la invertibilidad a partir de $\{2,1,1\}$ se tiene del análisis de resolubilidad y los mapas $\Phi_{1}$ : 


$$
\mathrm{y}_{1}=\rho(\mathrm{m}, \mathrm{v})
$$

$\dot{y}_{1}=\frac{\partial \rho}{\partial m} f_{m}+\frac{\partial \rho}{\partial V} f_{v}$

$y_{1}=\rho(m, s)$

$\dot{y}_{1}=\frac{\partial \rho}{\partial m} f_{m}+\frac{\partial \rho}{\partial s} f_{s}$

y para $\{1,2,1\}$

$\mathrm{y}_{2}=\mathrm{T}$

$\dot{\mathrm{y}}_{2}=\mathrm{f}_{\mathrm{T}}$

(Ellis y col.)

(Mutha y col.)

considerando para (5.3b) sólo la dinámica del monómero se obtiene $\dot{y}_{1} \approx \frac{\partial \rho}{\partial m} f_{m}$

La ecuación (5.4) es resoluble para el iniciador, es decir, es posible incluir al mismo en la dinámica observable. La I-invertibilidad se cumple debido a dos propiedades físicas:

1) Disminución de la densidad con la concentración de monómero $(\partial \rho / \partial \mathrm{m}<0)$.

2) Aumento de la velocidad de polimerización con la concentración de iniciador $\left(\partial_{\mathrm{pol}} / \partial \mathrm{I}>0\right)$. con lo que el mapa $\Phi$ para $C_{2}$ es x-invertibles.

Para $C_{3}$, del capítulo 4 se sabe que (5.3c) es resoluble para el iniciador (por la dependencia de la velocidad de polimerización con respecto al iniciador), por lo que el mapa $\Phi$ para $C_{3}$ es $x-$ invertible.

Estabilidad robusta y exponencial de la dinámica no observable $\left(\mathrm{x}_{\mathrm{ul}}^{*}(\mathrm{t})\right)$

Las trayectorias de la dinámica no observable son RE-estables debido a que la dinámica del monómero no se encuentra entre las no observables:

$$
\begin{aligned}
& {\left[\begin{array}{llll}
\dot{I}^{*} & \ddot{s}^{*} & \ddot{\mu}_{0}^{*} & \ddot{\mu}_{2}^{*}
\end{array}\right]^{T}=\left[\begin{array}{llll}
f_{1} & f_{s} & f_{\mu_{0}} & f_{\mu_{2}}
\end{array}\right]_{\left[l^{\circ}, \rho(t), T(t), V(t), \mu_{0}, \mu_{i}^{*}\right]}^{T} \text { para } C_{1}}
\end{aligned}
$$

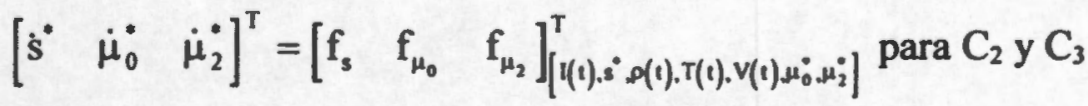

La dinámica del monómero con efecto gel es la única fuente de inestabilidad de las trayectorias del reactor. 
En resumen, para estas mediciones $(\rho, T$ y $V$ ) las trayectorias del reactor son $\mathrm{RE}$ - estimables con las siguientes estructuras de estimabilidad $S_{i}$ :

$$
\begin{aligned}
& S_{1}=\left\{(1,1,1), x_{I}=(m, T, V)\right\} \\
& S_{2}=\left\{(2,1,1), x_{I}=(I, m, T, V)\right\} \\
& S_{3}=\left\{(1,2,1), x_{I}=(I, m, T, V)\right\}
\end{aligned}
$$

Se verificará el desempeño de los estimadores $E_{1}$ y $E_{2}$ asociados con las estructuras de estimabilidad $S_{1}$ y $S_{2}$, en el entendido de que $S_{2}$ y $S_{3}$ son nominalmente equivalentes.

\section{Tiempos de asentamiento y factores de amortiguamiento}

Los tiempos de asentamiento empleados y los factores de amortiguamiento para las ganancias de los estimadores no lineales fueron los siguientes:

$$
\begin{aligned}
& \left\lfloor\left(\tau_{\mathrm{a}}\right)_{\mathrm{p}},\left(\tau_{\mathrm{a}}\right)_{\mathrm{V}}\right]=[125,50] \mathrm{min} \text { (Ellis y col.) } \\
& {\left[\left(\tau_{\mathrm{a}}\right)_{\mathrm{p}},\left(\tau_{\mathrm{a}}\right)_{\mathrm{T}}\right]=[100,75] \mathrm{min} \text { (Mutha y col.) }} \\
& \zeta_{\mathrm{\rho} / \mathrm{T} / \mathrm{N}}=0.71
\end{aligned}
$$

La relación de tiempos de asentamiento $\tau_{\rho} / \tau_{v}$ para el proceso de Ellis y col. es 2.5 , siendo la misma relación que la de Alvarez y López (1999), aunque por tratarse de un reactor en lote, para el tiempo de asentamiento del volumen se puede utilizar el de densidad. El tiempo de asentamiento de la densidad en este proceso se determinó mediante prueba y error. Para el reactor de Mutha y col., el tiempo de asentamiento de densidad es el mismo que el de la conversión $(\approx 100$ minutos, cuando se tiene el $98 \%$ de la conversión en estado estacionario) a $\overline{\mathrm{T}}$ y $\overline{\mathrm{V}}$. El tiempo de asentamiento de la dinámica de la temperatura es $\approx 75$ minutos, determinado a $\overline{\mathrm{I}}, \overline{\mathrm{m}}, \mathrm{y} \overline{\mathrm{V}}$. El factor de escalamiento en tiempo $s_{o}$ se hizo igual a 10, de acuerdo con la regla empírica de que “el estimador debe ser diez veces más rápido que el proceso" (Alvarez y López, 1999). 


\section{Reactor en lote}

En la figura 5.1 se presentan la conversión (a), peso molecular en número (b) y peso molecular en peso (c) experimentales (exp) y predichos por el modelo (M) para el reactor de Ellis y col. (1994), para la temperatura de $60^{\circ} \mathrm{C}$. La conversión evoluciona dentro del intervalo $(0-0.8)$, en tanto que los pesos moleculares en número y peso cambian dentro de los intervalos $(17000-28000)$ y (37000 - 52000), respectivamente. El modelo predice aceptablemente el peso molecular en peso, pero para el peso molecular en número se presenta un error sistemático en todo el tiempo de reacción y la conversión modelada diverge hacia los 50 minutos.

(a)

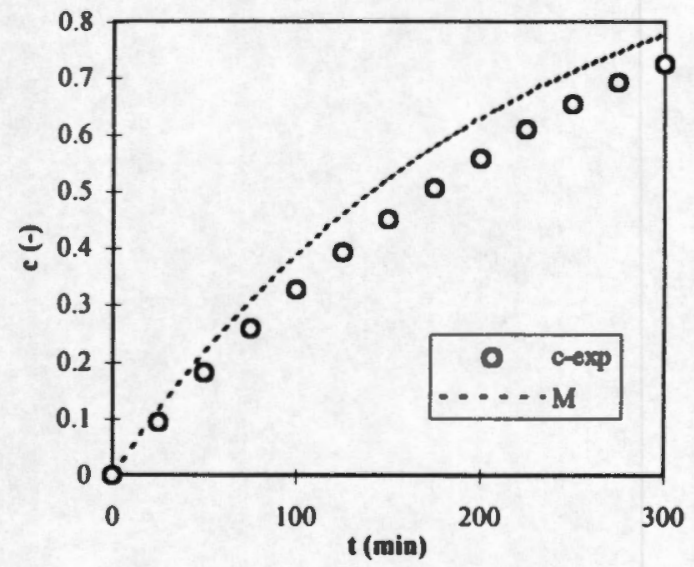

(b)

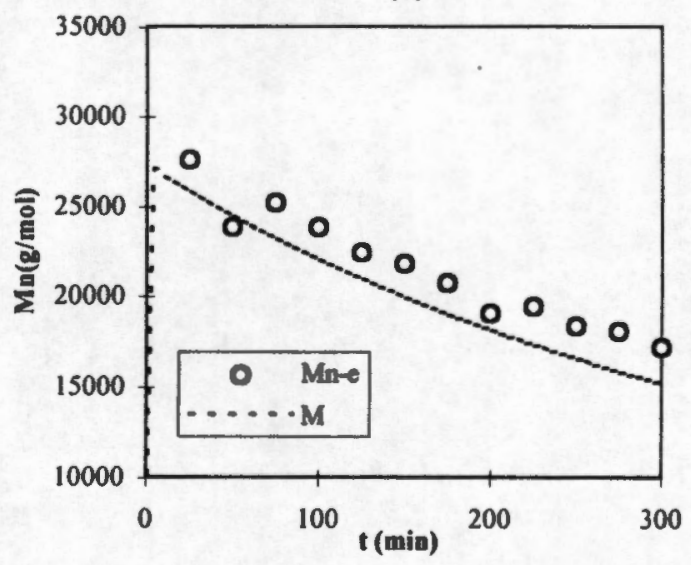

(c)

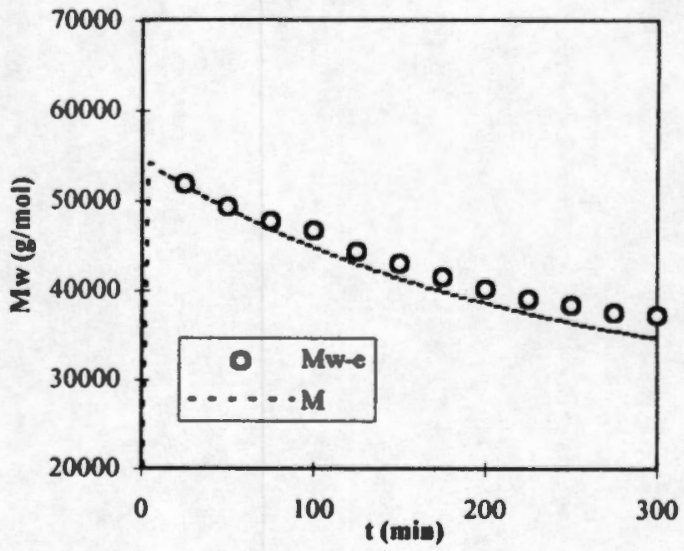

FIGURA 5.1. Comparación entre predicciones del modelo (- - ) y datos experimentales ( 0 ) (Ellis y col.): (a) Conversión, (b) peso molecular en número, (c) peso molecular en peso. 
En las figuras 5.2 y 5.3 se presenta el funcionamiento de los estimadores $E_{1}$ y $E_{2}$, asociados con las estructuras de estimabilidad $S_{1}$ y $S_{2}$.

La conversión estimada converge más rápidamente que los pesos moleculares porque está más ligada a la dinámica observable, mientras que los pesos moleculares están más relacionados con la dinámica no observable.

(a)

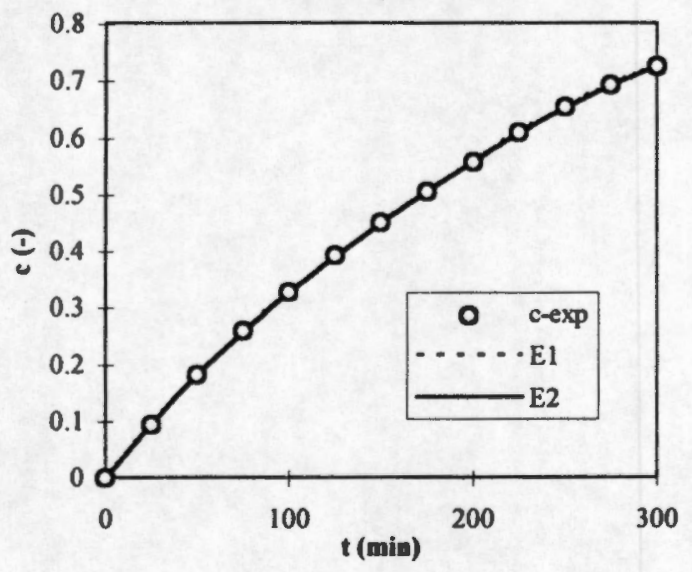

(b)

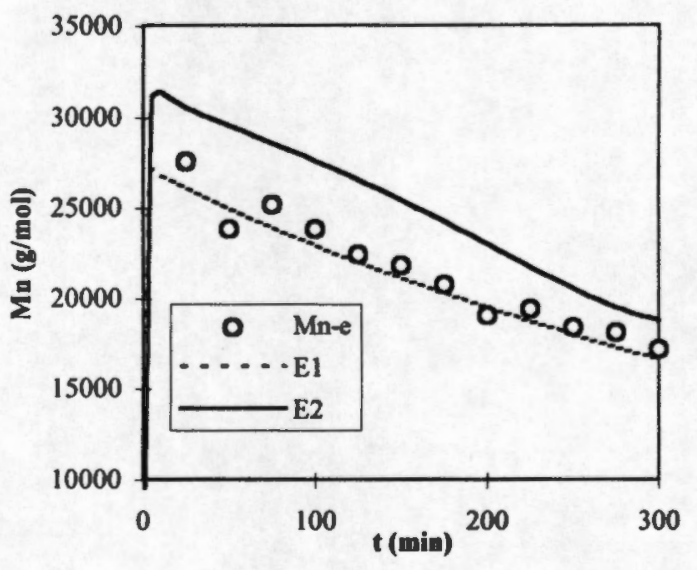

(c)

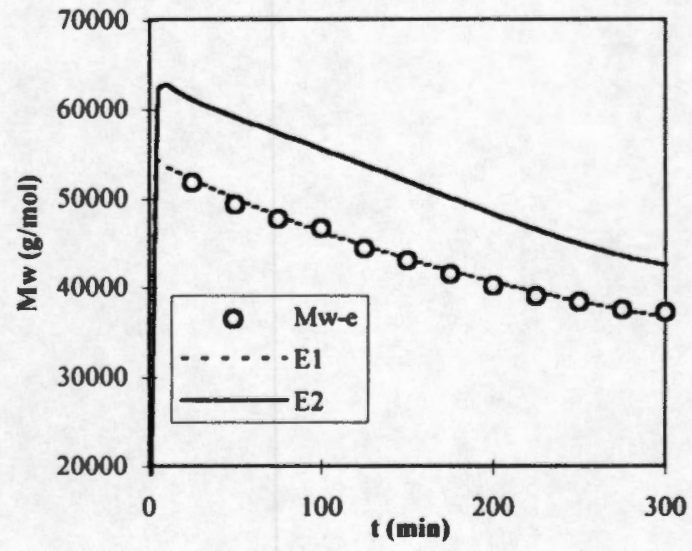

FIGURA 5.2. Comparación entre predicciones de estimadores $E_{1}, E_{2}$ y datos experimentales ( 0 ) (Ellis y col): (a) Conversión, (b) peso molecular en número, (c) peso molecular en peso.

El estimador $E_{2}$ presenta un comportamiento más oscilatorio en su desempeño, lo que se observa en la estimación de pesos moleculares en número y en peso. El estimador $E_{1}$ presenta en general 
un mejor desempeño; sus predicciones de pesos moleculares no presentan oscilaciones ni sesgo con respecto a los datos experimentales. El desempeño de los estimadores corrobora el fenómeno predicho por la teoría de estimación no lineal: a mayores índices de observabilidad se presenta mayor sensibilidad a errores de modelado y numéricos.

(a)

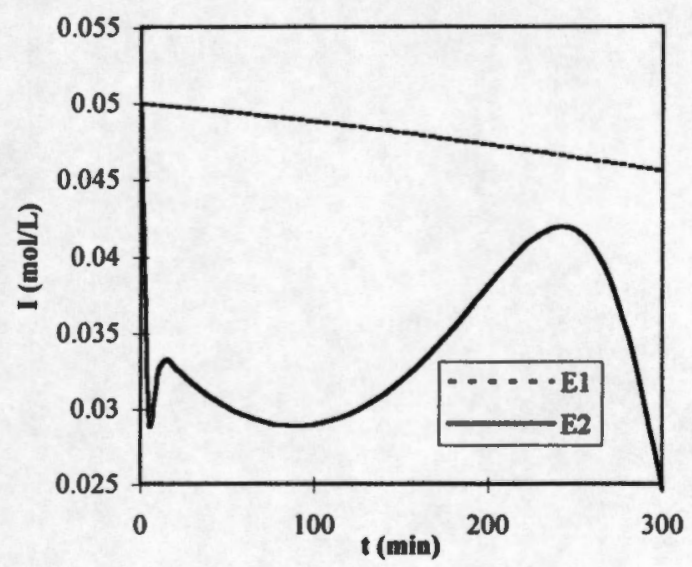

(b)

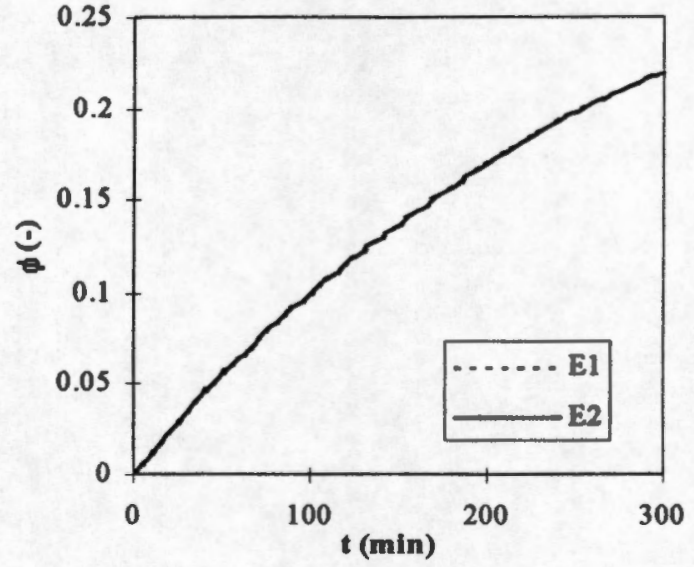

FIGURA 5.3. Comparación entre predicciones de estimadores $\mathrm{E}_{1}$, $\mathbf{E}_{2}$ (Ellis y col.): (a) Concentración de iniciador, (b) fracción masa de sólidos.

En la figura 5.3 se muestran las predicciones de los estimadores para la concentración de iniciador y la fracción masa de sólidos. La fracción masa está relacionada con la dinámica observable (siendo función de concentración de monómero y volumen) y ambos estimadores coinciden en la predicción. Por otra parte, la predicción de iniciador se degrada con $\mathrm{E}_{2}$; la inclusión de $I$ en la dinámica observable no lleva a un mejor funcionamiento.

En resumen, para la estimación de conversión y pesos moleculares en número y en peso en un reactor en lote, el estimador construido a partir del conjunto de índices de observabilidad $\left\{\kappa_{\rho}, \kappa v\right\}$ $=\{1,1\}$ presenta mejor desempeño que el construido con $\left\{\kappa_{\rho}, \kappa_{v}\right\}=\{2,1\}$. El aumento de la dimensión de la dinámica observable (mediante la inclusión de la concentración de iniciador) no conduce en este caso a un mejor desempeño; éste se degrada por la propagación de errores numéricos y de modelado. 


\section{Reactor continuo}

En la figura 5.5 se presentan la evolución de la conversión (a) y peso molecular en peso (b) experimentales (exp) y predichos por el modelo (M) para el reactor de Mutha y col. (1997), para la secuencias en escalón de temperatura y flujo de iniciador, mostradas en la figura 5.4. La conversión evoluciona dentro del intervalo $(0-0.65)$, en tanto que el peso molecular en peso cambia dentro del intervalo (20000 - 90000). Se observa que el proceso exhibe cambios drásticos en conversión y peso molecular como resultado de los cambios de temperatura y flujo de iniciador. Existe un comportamiento ruidoso de la conversión que también existe en la densidad.

(a)

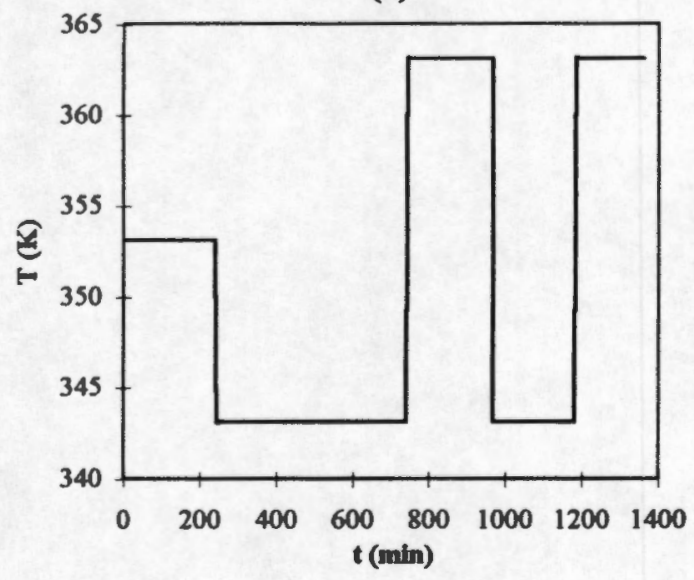

(b)

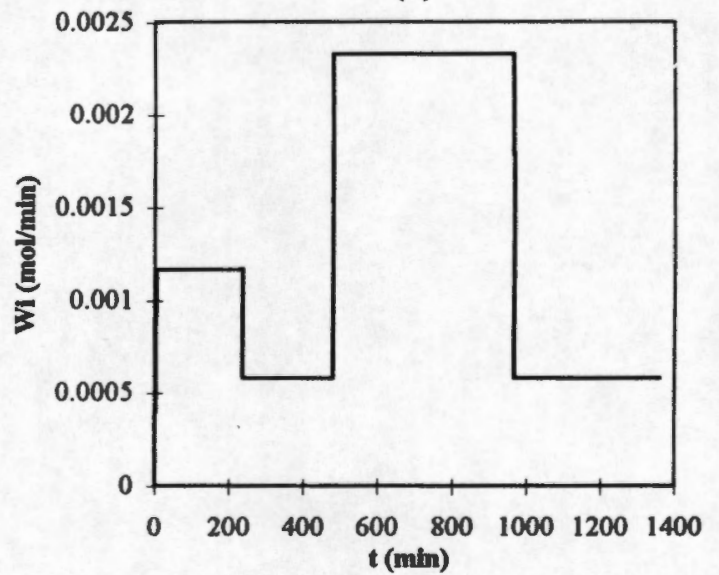

FIGURA 5.4. Secuencias de temperatura (a) y flujo de iniciador (b) en un reactor continuo (Mutha y col.)

(a)

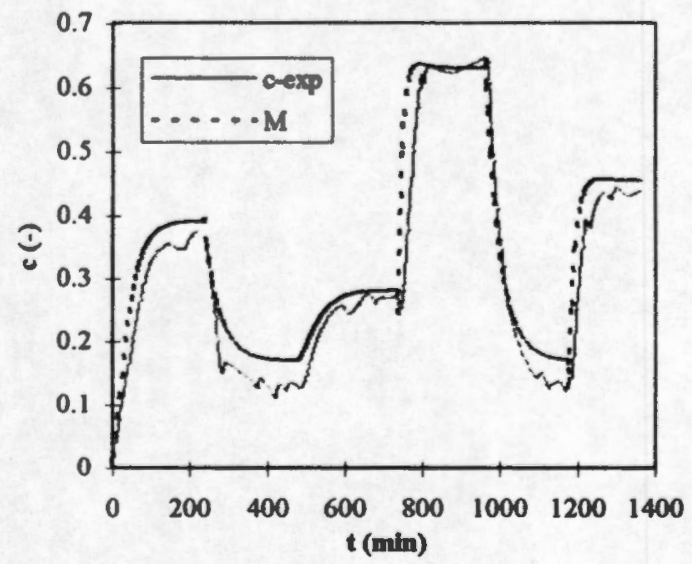

(b)

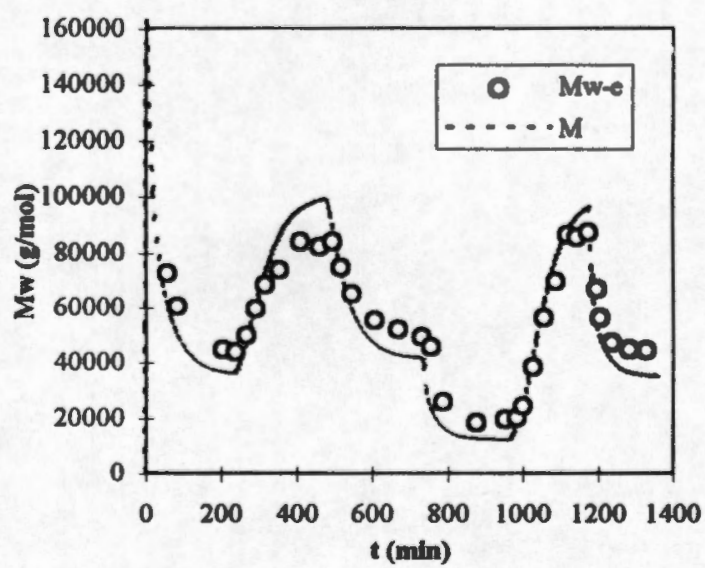

FIGURA 5.5. Comparación entre predicciones del modelo (M) y datos experimentales (Mutha y col.): (a) Conversión, (b) peso molecular en peso. 
La implementación de los estimadores con las condiciones reportadas por Ellis y col. (1994) muestra que el mejor desempeño se obtiene con índice de observabilidad igual a 1 para densidad (sin inclusión de iniciador en la dinámica observable). Con las condiciones de Mutha y col. (1997), el mejor desempeño también se obtiene con índice igual a 1 (figura 5.6). La estimación de la conversión es similar en ambos casos $\left(E_{1}\right.$ y $\left.E_{2}\right)$ : se presentan sesgos mayores en los tiempos en que el proceso tiene cambios en escalón simultáneos de temperatura y flujo de iniciador (239 y 965 minutos). Tales sesgos ya se presentan en la predicción de la conversión por el modelo; la acción de los estimadores no alcanza a eliminar el sesgo. El comportamiento ruidoso en la conversión se debe a que la densidad tiene el comportamiento de la misma clase, el cual afecta a la dinámica observable.

La estimación de peso molecular en peso presenta nuevamente un comportamiento oscilatorio con $E_{2}$, al igual que el estudio con el reactor en lote; tal comportamiento se acentúa en los tiempos en que existe cambios simultáneos de temperatura y flujo de iniciador.

En este proceso nuevamente la conversión estimada converge más rápidamente que el peso molecular en peso; esta rapidez en la convergencia se explica porque la conversión se encuentra más relacionada con la dinámica observable, mientras que el peso molecular con la no observable.

(a)

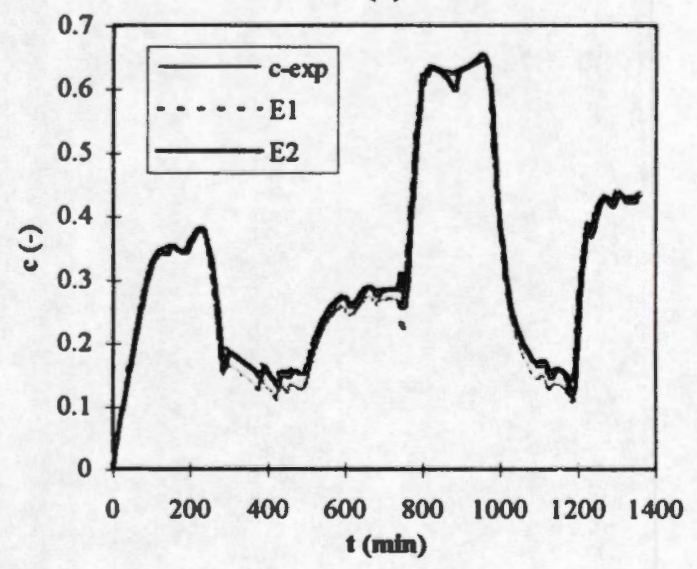

(b)

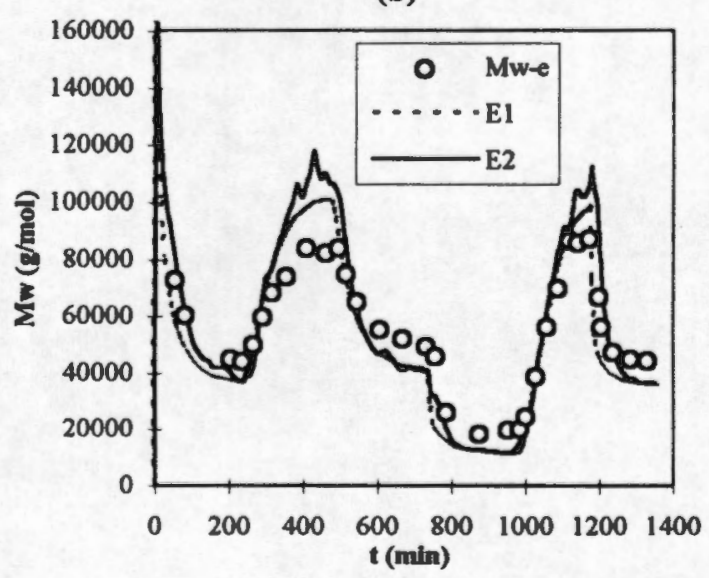

FIGURA 5.6. Comparación entre predicciones de estimadores $E_{1}, E_{2}$ y datos experimentales (Mutha y col.): (a) Conversión, (b) peso molecular en peso. 
(a)

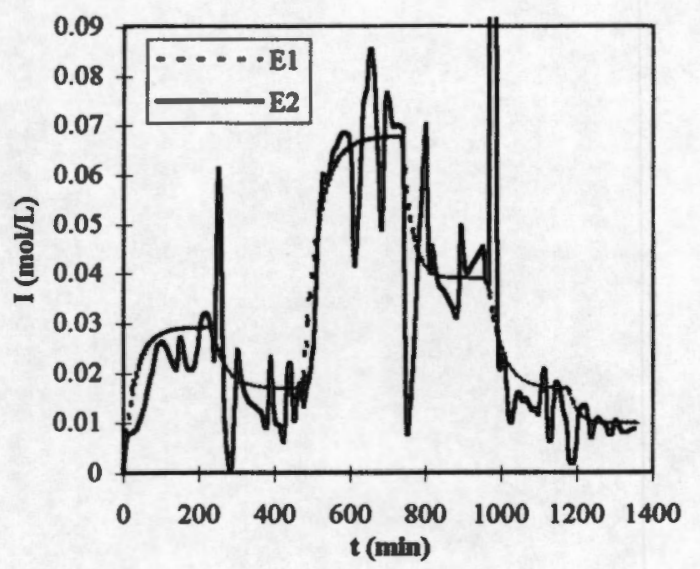

(b)

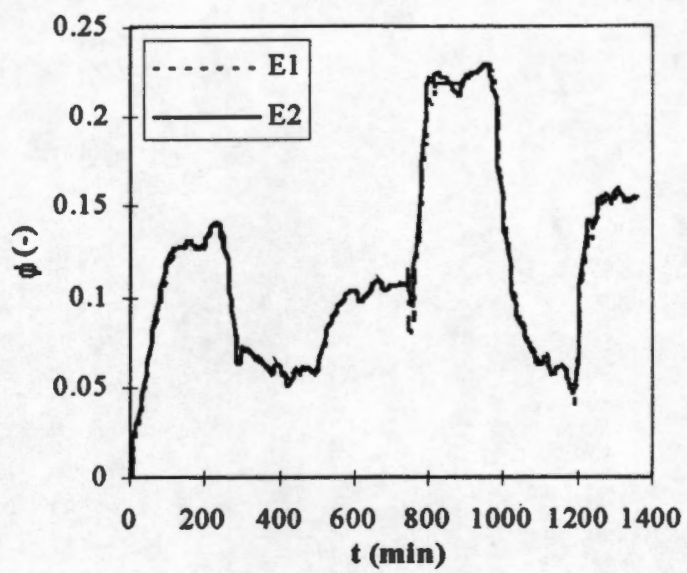

(c)

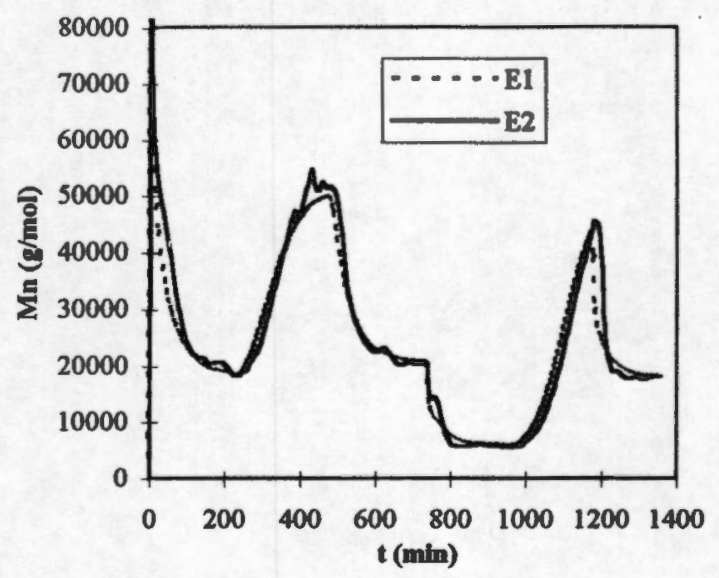

FIGURA 5.7. Comparación entre prediceiones de extimadores $\mathbf{E}_{1}, \mathbb{E}_{2}$ (Mutha y col.):

(a) Concentración de iniciador, (b) fracción masa de sólidos, (c) peso molecular en número.

La predicción de concentración de iniciador, fracción masa de sólidos y peso molecular en número se obtienen resultados similares al estudio con el reactor de Ellis y col. En la fracción masa de sólidos ambos estimadores coinciden en la predicción; la fracción masa está relacionada con la dinámica observable. La concentración de iniciador predicha por $\mathrm{E}_{2}$ presenta oscilaciones violentas; la inclusión de $I$ en la dinámica observable no conduce a un mejor funcionamiento. La predicción de peso molecular en número tiene las mismas tendencias que la del peso molecular en peso: la estimación con $\mathrm{E}_{2}$ tiene un comportamiento oscilatorio en ambos pesos moleculares.

En resumen, en la estimación de conversión y peso molecular en peso en un reactor continuo, el estimador construido a partir de $\left\{\kappa_{\rho,} \kappa_{T}\right\}=\{1,1\}$ presenta mejor desempeño que el construido con 
$\left\{\kappa_{\rho,}, \kappa_{T}\right\}=\{2,1\}$, al igual que el análisis realizado con el reactor en lote; la inclusión de la concentración de iniciador en la dinámica observable no conduce a un mejor desempeño.

Por otra parte, se corrobora la suposición realizada en el análisis de estimabilidad: la consideración de índices de observabilidad menores o iguales a 2 para evitar la propagación excesiva de errores numéricos y de modelado producto de la presencia de derivadas de alto orden en las matrices no lineales de los estimadores.

\section{Estimación con dinámica térmica y ruido en temperatura}

En el caso anterior se estudió la estimación con ruido en la densidad (también manifestado en la conversión); en este caso se estudiará también considerando ruido en temperatura. En la figura 5.8 se presenta la estimación de temperatura, conversión y peso molecular en peso cuando se emplean mediciones ruidosas de temperatura, tal como se reporta en el trabajo de Mutha y col. El ruido fue inyectado a intervalos de tiempo de 1 minuto, usando variables Gaussianas aleatorias, con una desviación estándar de $0.2 \mathrm{~K}$. La ecuación diferencial correspondiente a la temperatura se modeló de la siguiente forma:

$$
T=\beta r_{p}-Q+\frac{q_{e}}{V} \frac{\rho_{e}}{\rho}\left(T_{e}-T\right)
$$

donde $Q$ representa el calor intercambiado. La ecuación diferencial original para la temperatura es:

$$
\dot{T}=\beta r_{p}-\gamma\left(T-T_{c}\right)+\frac{q_{e}}{V}\left(\frac{1-\varepsilon_{m} m_{e}-\varepsilon_{s} s_{e}}{1-\varepsilon_{m} m-\varepsilon_{s} s}\right)\left(T_{e}-T\right)
$$

Obsérvese que $\gamma\left(T-T_{c}\right)$ se ha sustituido por $Q$. La expresión para $\gamma$ es (apéndice B):

$$
\gamma\left(\mathrm{m}, \mathrm{s}, \mathrm{T}, \mathrm{T}_{\mathrm{c}}, \mathrm{V}\right)=\frac{\mathrm{h}\left(\mathrm{m}, \mathrm{s}, \mathrm{T}, \mathrm{T}_{\mathrm{c}}\right) \mathrm{A}(\mathrm{V})}{\mathrm{V} \rho(\mathrm{m}, \mathrm{s}) \mathrm{C}_{\mathrm{p}}}
$$

Debido a que en el trabajo de Mutha y col. no se reportan diversos parámetros y dimensiones del reactor, el cálculo de se afronta mediante la consideración de un estado adicional $(Q)$.

Como puede esperarse, se verifica una mayor propagación del ruido en temperatura y conversión, ya que son las salidas que más dependientes de la dinámica observable La estimación de temperatura tiene una rápida convergencia, pero se presentan disparos en la estimación cuando existen grandes cambios en escalón de la temperatura del proceso (a 739, 965 y 1179 minutos). 
(a)

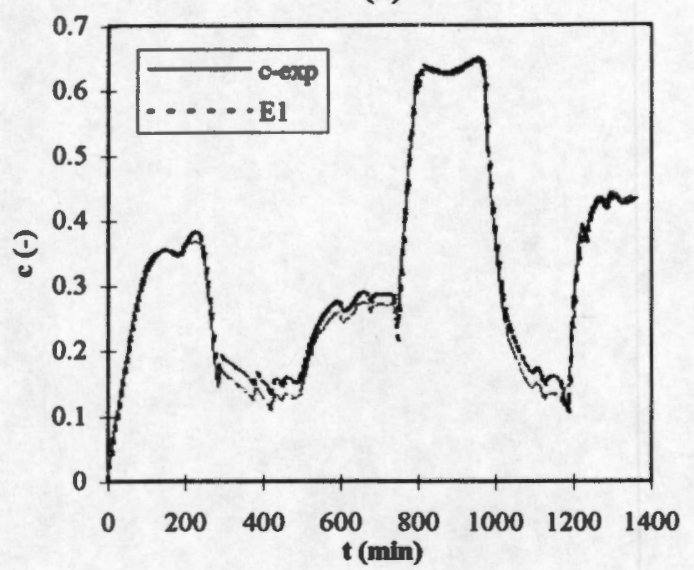

(b)

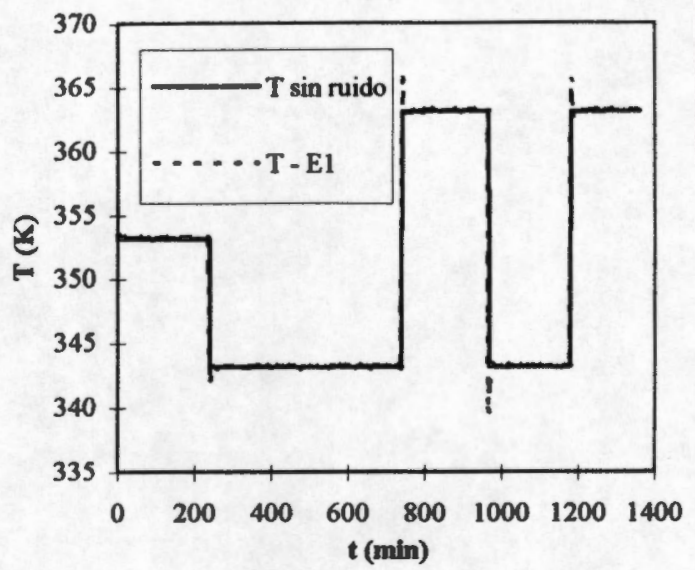

(c)

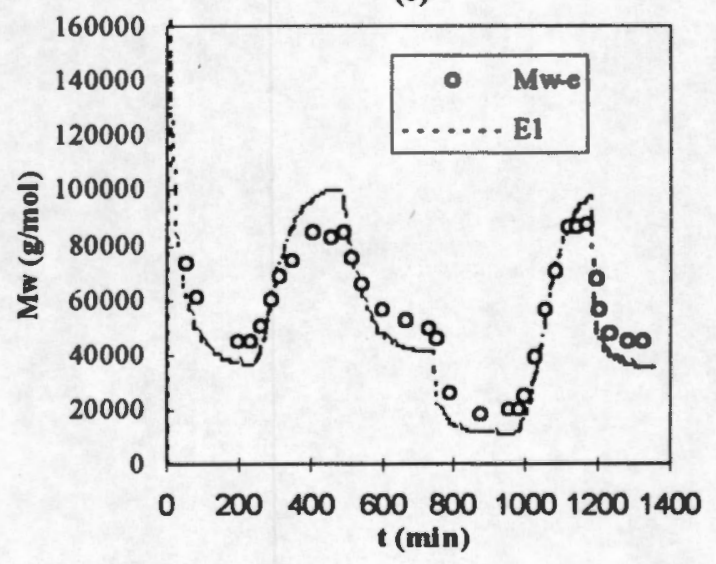

FIGURA 5.8. Comparación entre predicciones de estimador $\mathbf{E}_{1}$ y datos experimentales (Mutha y col.):

(a) Conversión, (b) temperatura, (c) peso molecular en peso.

El peso molecular, relacionado con la dinámica no observable, no resulta afectado por el ruido en temperatura.

En la figura 5.9 se presentan las predicciones para la concentración de iniciador, fracción masa de sólidos, peso molecular en número y calor intercambiado. La fracción masa de sólidos y el calor intercambiado, al estar relacionados con la dinámica observable, muestran comportamiento ruidoso en la estimación. La concentración de iniciador y el peso molecular en número no resultan afectados, al estar ligados a la dinámica no observable. 
(a)

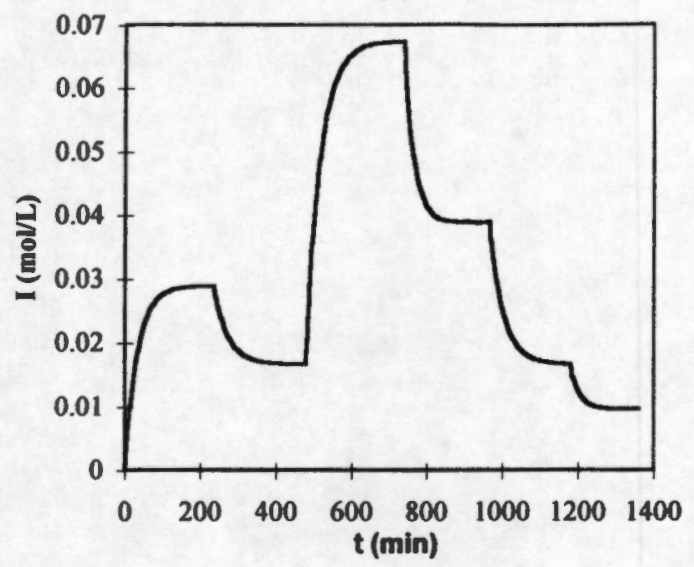

(c)

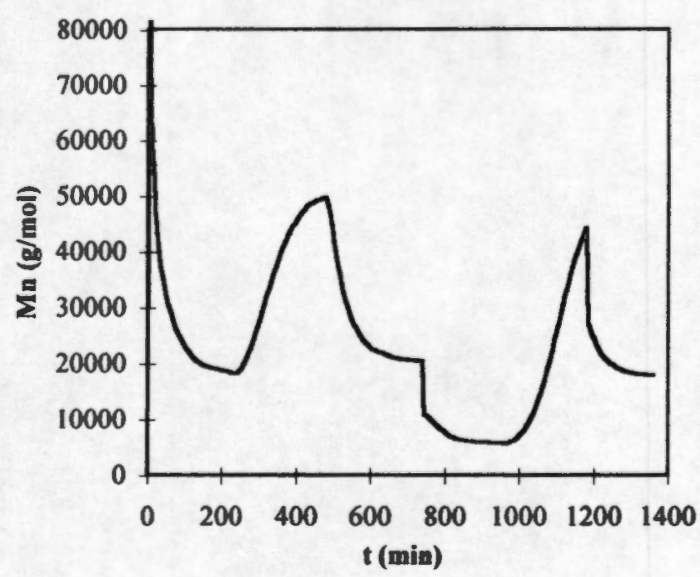

(b)

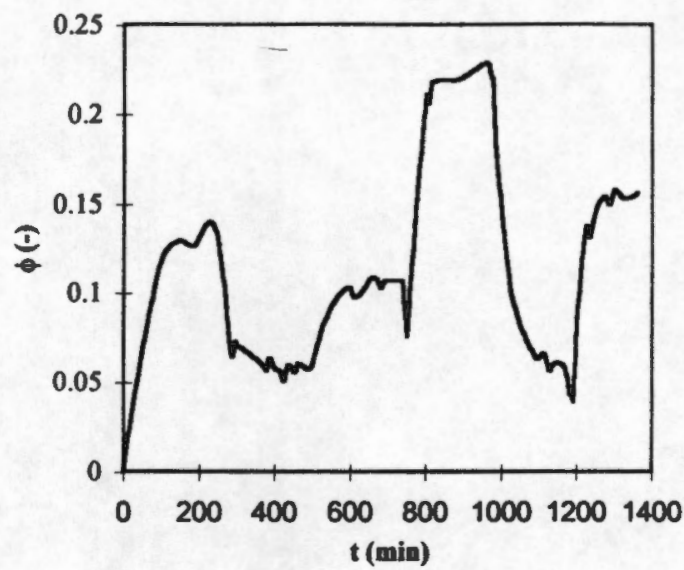

(d)

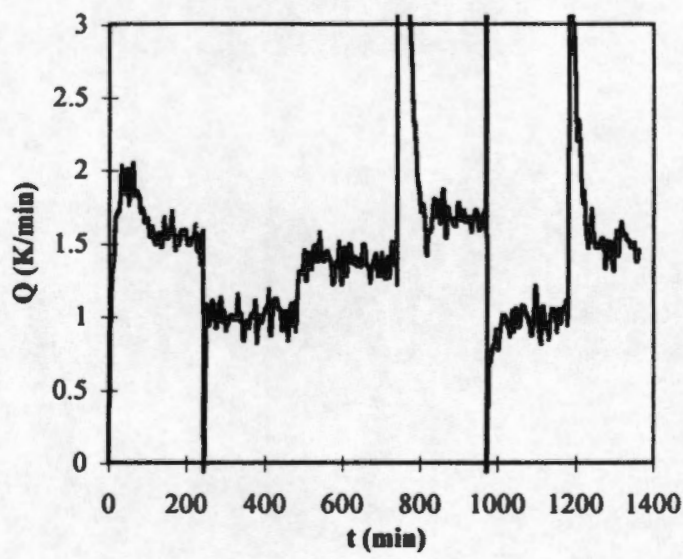

FIGURA 5.9. Predicciones del estimador $E_{1}$ (Mutha y col.):

(a) Concentración de iniciador, (b) fracción masa de sólidos,

(c) peso molecular en número, (d) calor intercambiado.

Como resultado global del capítulo, se determinó que tanto para un reactor en lote (con mediciones de densidad y volumen), como para un reactor continuo (con mediciones de densidad y temperatura) el estimador construido con índice de observabilidad igual a 1 para densidad presenta un mejor desempeño en comparación con índice igual a 2. Aunque ambos estimadores manifiestan comportamiento similar en la predicción de propiedades ligadas con la dinámica observable (conversión, fracción masa de sólidos), la estimación con índice 1 de pesos moleculares es mejor que la de índice 2. El aumento de índice 1 a 2 implica que el iniciador se incluye en la dinámica observable; la inclusión de iniciador en la misma no conduce a un mejor desempeño. El desempeño se degrada por la propagación de errores de modelado (ya que inexorablemente se 
tiene un modelo aproximado). Por otra parte, aunque la presencia de ruido en las mediciones afecta a la dinámica observable, las conclusiones se mantienen. 


\section{Capítulo VI. Conclusiones}

En este proyecto de investigación de tesis de maestria se estudió el problema de estimación para reactores de homopolimerización en solución (vía radicales libres), considerando que la polimerización en masa es un caso particular. Como casos de estudio se emplearon dos sistemas experimentales reportados en literatura. El estudio del problema de estimación abarcó el análisis de la resolubilidad del problema, la construcción y sintonización de los estimadores, y verificación del desempeño de los estimadores mediante el uso de mediciones experimentales (densidad, temperatura, volumen) y comparación de las estimaciones con datos reportados (conversión, pesos moleculares). El empleo de mediciones experimentales para la validación del estudio es una de las principales aportaciones de este trabajo.

La resolubilidad se analizó en función de las mediciones $(m / \rho / \eta, T, V$ y los conjuntos posibles de índices de observabilidad. Se concluyó que en el proceso estudiado, las trayectorias del reactor son robusta y exponencialmente estimables para ciertos conjuntos de indices de observabilidad. Asimismo se determinó analíticamente que los estados de la dinámica observable son la concentración de monómero, la temperatura, el volumen y la concentración de iniciador; la concentración de solvente puede incluirse en la dinámica observable mediante la incorporación de una medición adicional (si se emplea densidad, la medición adicional puede ser el índice de refracción) o bien si el reactor opera en régimen de efecto gel o si la reacción de transferencia a solvente es significativa. El estudio del desempeño se realizó para un reactor en lote (con mediciones de densidad y volumen), y un reactor continuo (con mediciones de densidad y temperatura). El estimador construido con índice de observabilidad igual a 1 para densidad presenta un mejor desempeño en comparación con el construido con índice igual a 2. La mejoría del desempeño se manifiesta principalmente en la estimación de propiedades ligadas a la dinámica no observable (pesos moleculares); ambos estimadores tienen comportamiento similar en la predicción de propiedades ligadas con la dinámica observable (conversión, fracción masa de sólidos. El aumento de índice 1 a 2 implica que el iniciador se incluye en la dinámica observable; sin embargo, la inclusión de iniciador en la misma no conduce a un mejor desempeño. El desempeño se degrada por la propagación de errores de modelado (ya que inexorablemente se 
tiene un modelo aproximado).

Como conclusión general se deriva que la estructura de estimabilidad afecta el desempeño de los estimadores para este tipo de procesos (homopolimerización en solución vía radicales libres); cada proceso requiere de un estudio de estimabilidad específico debido a las diferencias entre modelo, parámetros y condiciones de operación. Los índices de observabilidad altos favorecen la propagación de errores paramétricos y numéricos para este tipo de procesos, especialmente cuando existe cierta incertidumbre en el modelo.

Por otra parte, la inclusión del solvente en la dinámica observable depende de una medición adicional, del efecto gel o bien de una mayor velocidad de transferencia de cadena. 


\section{Referencias}

Alvarez, J. "A Robust State Estimation Design for Nonlinear Plants" Am. Contr. Conf . 5, 3058 (1997)

Alvarez, J., y López, T. "Robust Dynamic State Estimation of Nonlinear Plants", AIChE J. 45 (1) 107 (1999).

Alvarez, J., Suárez, R., y Sánchez, C. "Nonlinear decoupling control of free-radical polymerization continuous stirred tank reactors", Chem. Engng. Sci. 45 (11) 3341 (1990)

Bondy, F. y Lippa, S. "Heat Transfer in Agitated Vessels", Chem. Engng. April, 62 (1983).

Chiu, W. Y., Carratt, G.M., y Soong, D.S. “A Computer Model for the Gel Effect in Free-Radical Polymerization", Macromolecules. 16 (3) 348 (1983).

Ciccarella, G., Dalla-Mora, M., y Germani, A. "A Luenberguer-Like Observer for Nonlinear Systems", Int. J. Control. 57 (3) 537 (1993)

Ellis, M.F., Taylor, T.W., Gonzalez V., y Jensen, K.F. "Estimation fo the Molecular Weight Distribution in Batch Polymerization", AIChE J. 34 (8) 1341 (1988).

Ellis, M.F., Taylor, T.W., y Jensen, K.F. "On-Line Molecular Weight Distribution Estimation and Control in Batch Polymerization", AIChE J. 40 (3) 445 (1994).

Gauthier, J. P., Hammouri, H., y Othman, S. "A Simple Observer for Nonlinear Systems. Applications to Bioreactors", IEEE TAC. 37 (6) 875 (1992).

Jazwinksi, A. H. Stochastic Processes and Filtering Theory, Academic Press, New York (1970). 
Krener, A., y Respondek, W. "Nonlinear Observers with Linerizable Error Dynamics", SIAM J. Control Optim., 23 (2), 197 (1985).

Krener, A. e Isidori, A. "Linearization by Output Injection and Nonlinear Observers", Syst. and Control Lett. 3, 47 (1983).

Kwakernaak, H., y Sivan, R. Linear Optimal Control Systems, Wiley \& Sons, New York (1972).

López T., y Alvarez, J. "State Estimation of a Continuous Solution Copolymer Reactor", Proc. IFAC ADCHEM Symp., p. 213 (1997).

Lorimer, J. W. "Refractive Index Increments of Polymers in Solution", Polymer. 13, (6) 274 (1972).

Mutha, R.K., Cluett, W.R., y Penlidis, A., T. "On-Line Nonlinear Model-Based Estimation and Control of a Polymer Reactor", AIChE J. 43 (11) 3042 (1997).

Odian, G. Principles of Polymerization, Wiley \& Sons, New York (1991).

Shuler, H., y Papadopoulou, S. "Real-Time Estimation of the Chain Length Distribution in a Polymerization Reactor - I. Comparison of Estimated and Measured Distribution Functions", Chem. Engng. Sci. 41 (10) 2681 (1986).

Slotine, J. J. E., Hedrick, J. K., y Misawa, E. A. "On Sliding Observers for Nonlinear Systems", J. Dyn. Syst. Meas. Control,. 109, 245 (1987)

Stephanopoulos, G. Chemical Process Control, Prentice Hall, Englewood Cliffs, NJ (1984).

Uribe , M. Los polímeros, Noriega Editores, México, D.F (1990). 


\section{Apéndice A. Vectores y matrices del estimador no lineal}

bd $\mathbf{M}$ := matriz $\mathbf{M}$ diagonal en bloques

$\Gamma_{\mathrm{u}}=\operatorname{bd}\left[\Gamma_{1}^{\mathrm{u}}, \ldots, \Gamma_{\mathrm{p}}^{\mathrm{u}}\right]$

$\underbrace{\Gamma_{i}^{u}}_{v_{i} \times v_{i}}=\left[\begin{array}{ccccc}0 & 1 & 0 & \cdots & 0 \\ 0 & 0 & \ddots & & \vdots \\ \vdots & & & 1 & 0 \\ 0 & 0 & \cdots & 0 & 1 \\ 0 & 0 & 0 & \cdots & 0\end{array}\right]$

$1 \leq \mathrm{i} \leq \mathrm{p}$

$\Delta_{\mathrm{u}}=\operatorname{bd}\left[\delta_{1}^{\mathrm{u}}, \ldots, \delta_{\mathrm{p}}^{\mathrm{u}}\right]$

$\underbrace{\delta_{\mathrm{i}}^{\mathrm{u}}}_{1 \mathrm{vv}_{\mathrm{i}}}=\left[\begin{array}{llll}1 & 0 & \cdots & 0\end{array}\right]$

$1 \leq \mathrm{i} \leq \mathrm{p}$

donde

$p=$ número de entradas exógenas $(u)$

$v_{i}=$ índice $i$ de exo-observabilidad

$\Pi_{\mathrm{o}}=\mathrm{bd}\left[\pi_{1}^{\mathrm{o}}, \ldots, \pi_{\mathrm{m}}^{\mathrm{o}}\right]$

$\underbrace{\pi_{i}^{0}}_{x_{i} \times 1}=\left[\begin{array}{c}0 \\ \vdots \\ 0 \\ 1\end{array}\right]$

$1 \leq \mathrm{i} \leq \mathrm{m}$

donde

$\boldsymbol{m}=$ número de salidas medidas $(y)$

$\kappa_{i}=$ indice $i$ de observabilidad 


\section{Apéndice B. Modelo de homopolimerización en solución por radicales libres}

\section{Esquema cinético}

Iniciación

$\mathrm{I} \stackrel{k_{4}}{\longrightarrow} 2 \mathrm{R}$

$\mathrm{R}+\mathrm{M} \stackrel{\boldsymbol{k}_{1}}{\longrightarrow} \mathrm{P}_{1}$

Propagación

$\mathrm{P}_{\mathrm{i}}+\mathrm{M} \stackrel{k_{\mathrm{p}}}{\longrightarrow} \mathrm{P}_{\mathrm{i}+1}+\left(-\Delta \mathrm{H}_{\mathrm{p}}\right)$

Terminación

$$
\begin{aligned}
& P_{i}+P_{j} \stackrel{k_{e}}{\longrightarrow} D_{i+j} \\
& P_{i}+P_{j} \stackrel{k_{m}}{\longrightarrow} D_{i}+D_{j}
\end{aligned}
$$

Transferencia de cadena a monómero

$$
\mathrm{P}_{\mathrm{i}}+\mathrm{M} \stackrel{\mathrm{k}_{\text {ad }}}{\longrightarrow} \mathrm{D}_{\mathrm{i}}+\mathrm{P}_{\mathrm{i}}
$$

Transferencia de cadena a solvente

$$
\begin{aligned}
& \mathrm{P}_{\mathrm{i}}+\mathrm{S} \stackrel{\mathrm{k}_{\mathrm{m}}}{\longrightarrow} \mathrm{D}_{\mathrm{i}}+\mathrm{S}^{*} \\
& \mathrm{~S}^{*}+\mathrm{M} \stackrel{\mathrm{k}_{\mathrm{m}}}{\longrightarrow} \mathrm{P}_{1}
\end{aligned}
$$

Transferencia de cadena a un agente de transferencia

$$
\begin{aligned}
& \mathrm{P}_{i}+\mathrm{W} \stackrel{\mathrm{k}_{\mathrm{m}}}{\longrightarrow} \mathrm{D}_{\mathrm{i}}+\mathrm{W}^{*} \\
& \mathrm{~W}^{*}+\mathrm{M} \stackrel{\mathrm{k}_{\mathrm{a}}}{\longrightarrow} \mathrm{P}_{1}
\end{aligned}
$$

Ecuaciones de balance de materia y energia (en un RCTA)

Iniciador

$$
\frac{d(V I)}{d t}=-V k_{d} I+q_{e} I_{e}-q I
$$


Monómero

$\frac{d(V M)}{d t}=-V k_{i} M R-V\left(k_{p}+k_{f M}\right) M \lambda_{0}-V k_{a} M W *-V k_{a s} M S *+q_{e} M_{e}-q M$

Solvente

$\frac{d(V S)}{d t}=-V k_{f s} S \lambda_{0}+q_{e} S_{e}-q S$

Agente de transferencia

$\frac{d(V W)}{d t}=-V k_{\text {fin }} W \lambda_{0}+q_{e} W_{e}-q W$

Temperatura de reactor

$\frac{d\left(V \rho C_{p} T\right)}{d t}=V\left(-\Delta H_{p}\right) k_{p} M \lambda_{0}-A h\left(T-T_{c}\right)+q_{e} \rho_{e} C_{p e} T_{e}-q \rho C_{p} T$

Temperatura de camisa de enfriamiento

$\frac{d\left(V_{c} \rho_{c} C_{p c} T_{c}\right)}{d t}=A h\left(T-T_{c}\right)+q_{c} \rho_{c} C_{p c} T_{c e}-q_{c} \rho_{c} C_{p c} T_{c}$

Masa total

$$
\frac{d(V \rho)}{d t}=q_{e} \rho_{e}-q \rho
$$

Radical R (en lote)

$\frac{d(V R)}{d t}=2 V_{f} I-V_{d} R M$

Radical S* (en lote)

$\frac{d(V S *)}{d t}=V k_{f s} S \lambda_{0}-V k_{a s} M S *$

Radical W* (en lote)

$\frac{\mathrm{d}\left(\mathrm{VW}^{*}\right)}{\mathrm{dt}}=\mathrm{Vk}_{\mathrm{fin}} \mathrm{W}_{\lambda_{0}}-\mathrm{Vk}_{\mathrm{a}} \mathrm{MW} \mathrm{W}^{*}$

Radicales $\mathbf{P}_{\mathbf{i}}$ (en lote)

$$
\begin{aligned}
\frac{d\left(V P_{1}\right)}{d t} & =V k_{i} M R-V\left(k_{p}+k_{m}\right) M P_{1}-V\left(k_{r c}+k_{t d}\right) P_{1} \lambda_{0}-V k_{f m} P_{1} W+V k_{m M} M \lambda_{0}-V k_{f s} S P_{1} \\
& +V k_{a} M W^{*}+V k_{a s} M S^{*}
\end{aligned}
$$




$$
\begin{aligned}
& \frac{d\left(V P_{2}\right)}{d t}=V k_{p} M P_{1}-V\left(k_{p}+k_{f M}\right) M P_{2}-V\left(k_{t c}+k_{t d}\right) P_{2} \lambda_{0}-V k_{f m} P_{2} W-V k_{f s} P_{2} S \\
& \frac{d\left(V P_{3}\right)}{d t}=V k_{p} M P_{2}-V\left(k_{p}+k_{f M}\right) M P_{3}-V\left(k_{t c}+k_{t d}\right) P_{3} \lambda_{0}-V k_{f m} P_{3} W-V k_{f s} P_{3} S
\end{aligned}
$$

y en general...

$$
\frac{d\left(V P_{i}\right)}{d t}=\underset{i>1}{V k_{p}} M P_{i-1}-V\left(k_{p}+k_{p M}\right) M P_{i}-V\left(k_{t c}+k_{t d}\right) P_{i} \lambda_{0}-V k_{f f m} P_{i} W-V k_{t s} P_{i} S
$$

Radicales $D_{i}$ (en lote)

$$
\begin{aligned}
& \frac{d\left(V D_{1}\right)}{d t}=V k_{f f m} P_{1} W+V k_{t d} P_{1} \lambda_{0}+V k_{i M} P_{1} M+V k_{f s} P_{1} S \\
& \frac{d\left(V D_{2}\right)}{d t}=V k_{f m} P_{2} W+V k_{t d} P_{2} \lambda_{0}+V k_{t c} P_{1} P_{1}+V k_{f M} P_{2} M+V k_{f s} P_{2} S \\
& \frac{d\left(V D_{3}\right)}{d t}=V k_{f m} P_{3} W+V k_{t d} P_{3} \lambda_{0}+V k_{t c} P_{1} P_{2}+V k_{M M} P_{3} M+V k_{f s} P_{3} S \\
& \frac{d\left(V D_{4}\right)}{d t}=V k_{\text {ffm }} P_{4} W+V k_{t d} P_{4} \lambda_{0}+V k_{t c}\left(P_{1} P_{3}+P_{2} P_{2}\right)+V k_{g M} P_{4} M+V k_{f s} P_{4} S \\
& \frac{d\left(V D_{5}\right)}{d t}=V k_{f f m} P_{5} W+V k_{c t} P_{5} \lambda_{0}+V k_{t c}\left(P_{1} P_{4}+P_{2} P_{3}\right)+V k_{g M} P_{5} M+V k_{f s} P_{5} S \\
& \frac{d\left(V D_{6}\right)}{d t}=V k_{\text {fim }} P_{6} W+V k_{t d} P_{6} \lambda_{0}+V k_{t c}\left(P_{1} P_{5}+P_{2} P_{4}+P_{3} P_{3}\right)+V k_{f M} P_{6} M+V k_{f s} P_{6} S
\end{aligned}
$$

$y$ en general...

i : par

$$
\frac{d\left(V D_{i}\right)}{d t}=V k_{f m} P_{i} W+V k_{t d} P_{i} \lambda_{0}+V k_{t c} \sum_{j=1}^{i / 2} P_{j} P_{i-j}+V k_{f M} P_{1} M+V k_{f s} P_{1} S
$$

i : impar

$$
\frac{d\left(V D_{i}\right)}{d t}=V k_{f m} P_{i} W+V k_{t d} P_{i} \lambda_{o}+V k_{k c} \sum_{j=1}^{(i-1) / 2} P_{j} P_{i-j}+V k_{m M} P_{1} M+V k_{f s} P_{1} S
$$




\section{Tasas de cambio de los estados}

Iniciador

$$
I=-r_{I}(I, T)+\left\{\varepsilon_{m} r_{p o l}(I, m, s, W, T)+\varepsilon_{s} r_{s o l}(I, m, s, W, T)\right\} I+\frac{\left(i_{e}-q_{e} I\right)}{V}:=f_{I}
$$

Solvente (adimensional)

$$
s=-r_{s o l}(I, m, s, W, T)+\left\{\varepsilon_{m} r_{p o l}(I, m, s, W, T)+\varepsilon_{s} r_{s o l}(I, m, s, W, T)\right\}+\frac{q_{e}}{V}\left(s_{e}-s\right):=f_{s}
$$

Agente de transferencia

$$
W=-r_{W}(I, m, s, W, T)+\left\{\varepsilon_{m} r_{p o l}(I, m, s, W, T)+\varepsilon_{s} r_{s o l}(I, m, s, W, T)\right\} W+\frac{q_{e}}{V}(W,-W):=f_{W}(B 3
$$

Monómero (adimensional)

$$
\mathrm{m}=-\mathrm{r}_{\mathrm{pol}}(\mathrm{I}, \mathrm{m}, \mathrm{s}, \mathrm{W}, \mathrm{T})+\left\{\varepsilon_{\mathrm{m}} \mathrm{r}_{\mathrm{pol}}(\mathrm{I}, \mathrm{m}, \mathrm{s}, \mathrm{W}, \mathrm{T})+\varepsilon_{\mathrm{s}} \mathrm{r}_{\mathrm{sol}}(\mathrm{I}, \mathrm{m}, \mathrm{s}, \mathrm{W}, \mathrm{T})\right\}_{\mathrm{m}}+\frac{\mathrm{q}_{\mathrm{e}}}{\mathrm{V}}\left(\mathrm{m}_{\mathrm{e}}-\mathrm{m}\right):=\mathrm{f}_{\mathrm{m}}
$$

Temperatura

$$
T=\beta(m, s) r_{p}(I, m, s, W, T)-\gamma(m, s, T, V)\left(T-T_{c}\right)+\frac{q_{e}}{V}\left(\frac{1-\varepsilon_{m} m_{e}-\varepsilon_{s} s_{e}}{1-\varepsilon_{m} m-\varepsilon_{s} s}\right)\left(T_{e}-T\right):=f_{T}
$$

Volumen

$$
V=-\left\{\varepsilon_{m} r_{p o l}(I, m, s, W, T)+\varepsilon_{s} r_{\text {sol }}(I, m, s, W, T)\right\} V+q_{e}-q:=f_{V}
$$

Momento cero de cadenas inactivas

$$
\mu_{0}=r_{\mu 0}(I, m, s, W, T)+\left\{\varepsilon_{m} r_{p o l}(I, m, s, W, T)+\varepsilon_{s} r_{s o l}(I, m, s, W, T)\right\}_{\mu_{0}}+\frac{q_{e}}{V}\left(\mu_{0 e}-\mu_{0}\right):=f_{\mu 0}(B 3 . g)
$$

Momento uno de cadenas inactivas

$$
\mu_{1}=r_{\mu l}(I, m, s, W, T)+\left\{\varepsilon_{m} r_{p o l}(I, m, s, W, T)+\varepsilon_{s} r_{s o l}(I, m, s, W, T) \mu_{1}+\frac{q_{e}}{V}\left(\mu_{1 e}-\mu_{1}\right):=f_{\mu 1}(B 3 . h)\right.
$$

Momento dos de cadenas inactivas

$$
\mu_{2}=r_{\mu 2}(I, m, s, W, T)+\left\{\varepsilon_{m} r_{p a l}(I, m, s, W, T)+\varepsilon_{s} r_{s o l}(I, m, s, W, T)\right\}_{\mu_{2}}+\frac{q_{e}}{V}\left(\mu_{2 e}-\mu_{2}\right):=f_{\mu 2}(B 3 . i)
$$




\section{Tasas de reacción}

Disociación de iniciador

$$
\mathbf{r}_{\mathrm{I}}(\mathrm{I}, \mathrm{T})=\mathbf{k}_{\mathrm{d}}(\mathrm{T}) \mathrm{I}
$$

Transferencia a solvente

$$
r_{s o l}(I, m, s, W, T)=k_{f s}(T) s \lambda_{0}(I, m, s, W, T)
$$

Transferencia a agente de transferencia

$r_{W}(I, m, s, W, T)=k_{\text {ff }}(I, m, s, W, T) W \lambda_{0}(I, m, s, W, T)$

Propagación

$$
r_{p}(I, m, s, W, T)=k_{p}(I, m, s, W, T) m \lambda_{0}(I, m, s, W, T)
$$

Transferencia a monómero

$$
r_{m}(I, m, s, W, T)=k_{M}(T) m \lambda_{0}(I, m, s, W, T)
$$

Polimerización total

$$
\begin{aligned}
r_{p o t}(I, m, s, W, T) & =\frac{2 f_{d}}{M^{\circ}} r_{I}(I, T)+r_{p}(I, m, s, W, T)+r_{m}(I, m, s, W, T) \\
& +\frac{1}{M^{\circ}} r_{W}(I, m, s, W, T)+\frac{S^{\circ}}{M^{\circ}} r_{s o l}(I, m, s, W, T)
\end{aligned}
$$

Momento cero de cadenas inactivas

$$
r_{\mu 0}(I, m, s, W, T)=\left\{\begin{array}{l}
k_{t}(I, m, s, W, T) \lambda_{0}(I, m, s, W, T)+M^{\circ} k_{\text {BM }}(I, m, s, W, T) m \\
+S^{\circ} k_{f s}(I, m, s, W, T) s+k_{f m}(I, m, s, W, T) W
\end{array}\right\} \lambda_{0}
$$

Momento uno de cadenas inactivas

$$
r_{\mu 1}(I, m, s, W, T)=\left\{\begin{array}{l}
k_{f}\left(I, m, s, W, T h_{0}(I, m, s, W, T)+M^{\circ} k_{\text {fM }}(I, m, s, W, T) m\right. \\
+S^{\circ} k_{f s}(I, m, s, W, T) s+k_{f m}(I, m, s, W, T) W
\end{array}\right\} \lambda_{1}
$$

Momento dos de cadenas inactivas

$$
r_{\mu 2}(I, m, s, W, T)=\left\{\begin{array}{l}
k_{t}(I, m, s, W, T) \lambda_{0}(I, m, s, W, T)+M^{\circ} k_{\text {fM }}(I, m, s, W, T) m \\
+S^{\circ} k_{\text {fs }}(I, m, s, W, T) s+k_{\text {fm }}(I, m, s, W, T) W
\end{array}\right\} \lambda_{2}
$$




\section{Definición de variables y propiedades}

Momentos de cadenas vivas
$\lambda_{0}=\sum_{i=1}^{\infty} P_{i}$
$\lambda_{1}=\sum_{i=1}^{\infty} \mathrm{iP}_{\mathrm{i}}$
$\lambda_{2}=\sum_{i=1}^{\infty} i^{2} P_{i}$

Momentos de cadenas inactivas
$\mu_{0}=\sum_{i=1}^{\infty} D_{i}$
$\mu_{1}=\sum_{i=1}^{\infty} \mathrm{iD}_{\mathrm{i}}$
$\mu_{2}=\sum_{i=1}^{\infty} i^{2} D_{i}$

Concentraciones molares de monómero y solvente puros

$$
\mathrm{M}^{0}=\frac{\rho_{\mathrm{m}}^{0}}{\mathrm{PM}_{\mathrm{m}}} \quad \mathrm{S}^{\circ}=\frac{\rho_{\mathrm{s}}^{0}}{\mathrm{PM}_{\mathrm{s}}}
$$

Concentraciones adimensionales de monómero y solvente

$$
m=\frac{M}{M^{0}} \quad s=\frac{S}{S^{\circ}}
$$

Factores de contracción de monómero, solvente e iniciador

$$
\varepsilon_{\mathrm{m}}=1-\frac{\rho_{\mathrm{m}}^{0}}{\rho_{\mathrm{p}}^{0}} \quad \varepsilon_{\mathrm{s}}=1-\frac{\rho_{\mathrm{s}}^{0}}{\rho_{\mathrm{p}}^{0}} \quad \varepsilon_{\mathrm{l}}=1-\frac{\rho_{\mathrm{l}}^{0}}{\rho_{\mathrm{p}}^{0}}
$$

Densidad

$$
\begin{aligned}
& \rho(m, s)=\frac{\rho_{m}^{\circ}}{1-\varepsilon_{m}}\left(1-\varepsilon_{m} m-\varepsilon_{s} s\right) \\
& \rho(m, V)=\frac{\rho_{m}^{\circ}}{1-\varepsilon_{m}}\left(1-\varepsilon_{m} m-\varepsilon_{s} \frac{V_{s}}{V}-\varepsilon_{1} \frac{V_{I}}{V}\right) \quad \text { (Ellis y col., 1994) } \\
& \rho(m, s)=\frac{(1-s)\left(1-\varepsilon_{m}\right)}{(1-s)-\varepsilon_{m}(2-2 s-m)} \rho_{e} \quad \text { (Mutha y col., 1997) } \\
& \rho_{e}=m_{c} \rho_{m}^{\circ}+s_{e} \rho_{s}^{\circ} \text { (densidad del flujo de entrada) }
\end{aligned}
$$

Conversión

$$
c=\frac{1-m-s}{1-\varepsilon_{m} m-s}
$$




$$
\begin{aligned}
& c=\frac{\frac{w_{T}}{\rho}-\frac{w_{m}}{\rho_{m}^{0}}-\frac{w_{s}}{\rho_{s}^{0}}-\frac{w_{I}}{\rho_{I}^{0}}}{\frac{w_{m}}{\rho_{p}^{0}}-\frac{w_{m}}{\rho_{m}^{0}}} \\
& c=\frac{1-\varepsilon_{m}}{\varepsilon_{m}}\left(1-\frac{\rho_{\mathrm{e}}}{\rho}\right)
\end{aligned}
$$

Fracción masa de sólidos

$$
\begin{aligned}
& \phi=\frac{1-m-s}{1-\varepsilon_{m} m-\varepsilon_{s} s} \\
& \phi=\frac{1-m-\frac{V_{s}}{V_{T}}-\frac{V_{1}}{V_{T}}}{1-\varepsilon_{m} m-\varepsilon_{s} \frac{V_{s}}{V_{T}}-\varepsilon_{I} \frac{V_{I}}{V_{T}}}
\end{aligned}
$$

$\phi=1-m-s$

Pesos moleculares en número y en peso

$$
M_{n}=P_{m} \frac{\mu_{1}}{\mu_{0}} \quad M_{w}=P M_{m} \frac{\mu_{2}}{\mu_{1}}
$$

Polidispersidad

$$
\mathrm{Q}=\frac{\mathrm{M}_{\mathrm{W}}}{\mathrm{M}_{\mathrm{n}}}
$$

\section{Constantes de reacción}

Disociación

$k_{d}(T)=\exp \left(A_{D}-\frac{B_{D}}{T}\right)$

Propagación sin gel

$$
k_{p 0}(T)=\exp \left(A_{p 0}-\frac{B_{p 0}}{T}\right)
$$


Terminación sin gel

$$
k_{10}(T)=\exp \left(A_{t 0}-\frac{B_{t 0}}{T}\right)
$$

Transferencia a monómero

$$
k_{P M}(T)=\exp \left(A_{P M}-\frac{B_{P M}}{T}\right)
$$

Transferencia a solvente

$$
k_{f s}(T)=\exp \left(A_{f s}-\frac{B_{f B}}{T}\right)
$$

\section{Efecto gel}

Constante de propagación con gel

$k_{p}(I, m, s, W, T)=\frac{k_{p 0}(T)}{1+\left\{k_{p 0}(T) \theta_{p}(T) E(m, s, T) \lambda_{0}(I, m, s, W, T)\right\}}$

Constante de terminación con gel

$k_{t}(I, m, s, W, T)=\frac{k_{t 0}(T)}{1+\left\{k_{t 0}(T) \theta_{t}(I, W, T) E(m, s, T) h_{0}(I, m, s, W, T)\right\}}$

Constante de transferencia a agente con gel

$k_{\text {fim }}(I, m, s, W, T)=k_{\text {fmo }} k_{p}(I, m, s, W, T)$

Volumen libre

$$
\begin{aligned}
& E(m, s, T)=\exp \left[\frac{-2.3(m+s)}{A(T)+(m+s) B(T)}\right] \\
& A(T)=A_{A}-B_{A}\left(1-\frac{T}{T_{B}}\right)-C_{A}\left(1-\frac{T}{T_{B}}\right)^{2} \\
& B(T)=A_{B}-B_{B}\left(1-\frac{T}{T_{B}}\right)
\end{aligned}
$$


Tiempo de propagación

$$
\theta_{p}(T)=\exp \left(-A_{t}+\frac{B_{\varphi}}{T}\right)
$$

Tiempo de terminación

$$
\theta_{t}(I, W, T)=\exp \left(-A_{\mathfrak{u}}-B_{\mathfrak{u}} I-C_{\mathfrak{u}} W+\frac{D_{\mathfrak{u}}}{T}\right)
$$

Momento 0 de cadenas vivas

$$
\begin{aligned}
& \lambda_{0}(\mathrm{I}, \mathrm{m}, \mathrm{s}, \mathrm{W}, \mathrm{T})=\sqrt{\lambda_{0 s}(\mathrm{I}, \mathrm{T})+\lambda_{\mathrm{g}}^{2}(\mathrm{I}, \mathrm{m}, \mathrm{s}, \mathrm{W}, \mathrm{T})}+\lambda_{\mathrm{g}}(\mathrm{I}, \mathrm{m}, \mathrm{s}, \mathrm{W}, \mathrm{T}) \\
& \lambda_{0 \mathrm{~s}}(\mathrm{I}, \mathrm{T})=\frac{2 \mathrm{f}_{\mathrm{d}} \mathrm{k}_{\mathrm{d}}(\mathrm{T}) \mathrm{I}}{\mathrm{k}_{\mathrm{t} 0}(\mathrm{~T})}(\sin \text { gel })
\end{aligned}
$$

$\lambda_{\mathrm{g}}(\mathrm{I}, \mathrm{m}, \mathrm{s}, \mathrm{W}, \mathrm{T})=\mathrm{f}_{\mathrm{d}} \mathrm{k}_{\mathrm{d}}(\mathrm{T}) \mathrm{I}_{\mathrm{t}}(\mathrm{I}, \mathrm{W}, \mathrm{T}) \mathrm{E}(\mathrm{m}, \mathrm{s}, \mathrm{T})$ (intensidad de efecto gel)

Momentos 1 y 2 de cadenas vivas

$$
\lambda_{1}(I, m, s, W, T)=\frac{M^{\circ} r_{p o l}(I, m, s, W, T)}{k_{t}(I, m, s, W, T) \lambda_{0}(I, m, s, W, T)+M^{\circ} k_{m}(T) m+S^{\circ} k_{k_{\mathrm{m}}}(T) s+k_{\text {fm }}(I, m, s, W, T) W}
$$

$$
\lambda_{2}(I, m, s, W, T)=\left(1+\frac{2 M^{\circ} k_{p}(I, m, s, W, T) m}{k_{t}(I, m, s, W, T) \lambda_{0}(I, m, s, W, T)+M^{\circ} k_{M}(T) m+S^{\circ} k_{f s}(T) s+k_{f m}(I, m, s, W, T) W}\right) \lambda_{1}
$$

\section{Transferencia de calor}

Incremento adiabático de temperatura

$$
\beta(m, s)=\frac{\left(-\Delta H_{p}\right) M^{\circ}}{\rho(m, s) C_{p}}
$$

Calor intercambiado/capacitancia térmica

$$
\gamma\left(m, s, T, T_{c}, V\right)=\frac{h\left(m, s, T, T_{c}\right) A(V)}{V p(m, s) C_{p}}
$$


Coeficiente de transferencia de calor (Bondy y Lippa, 1983)

$h\left(m, s, T, T_{c}\right)=A_{h}\left(\frac{k}{D_{R}}\right)\left(\frac{L^{2} N \rho(m, s)}{\mu(m, s, T)}\right)^{B h}\left(\frac{C_{p} \mu(m, s, T)}{k}\right)^{C h}\left(\frac{\mu(m, s, T)}{\mu_{w}\left(m, s, T, T_{c}\right)}\right)^{D h}$

Viscosidad de la mezcla en el reactor (Alvarez y col. 1990)

$\mu(\mathrm{m}, \mathrm{s}, \mathrm{T})=\mathrm{A}_{\mu}(\mathrm{T}-273.15)^{-\mathrm{B} \mu} \mathrm{E}^{\mathrm{C} \mu}(\mathrm{m}, \mathrm{s}, \mathrm{T})$

Viscosidad a temperatura de la camisa (Alvarez y col. 1990)

$\mu_{w}\left(m, s, T, T_{c}\right)=A_{\mu}(T-273.15)^{-B \mu} E_{J}^{C \mu}\left(m, s, T_{c}\right)$

Volumen libre a temperatura de la camisa

$E_{J}\left(m, s, T_{c}\right)=\exp \left[\frac{-2.3(m+s)}{A_{J}\left(T_{c}\right)+(m+s) B_{J}\left(T_{c}\right)}\right]$

$A_{J}\left(T_{c}\right)=A_{A}-B_{A}\left(1-\frac{T_{c}}{T_{g}}\right)-C_{A}\left(1-\frac{T_{c}}{T_{g}}\right)^{2}$

$B_{J}\left(T_{c}\right)=A_{B}-B_{B}\left(1-\frac{T_{c}}{T_{B}}\right)$

Area de intercambio de calor

$$
A(V)=4 \frac{V}{D_{R}}+\frac{\pi D^{2}}{4}
$$


Valores utilizados en el modelo de polimerización y estimadores no lineales

Tabla B.1. Condiciones iniciales

\begin{tabular}{|l|c|c|}
\hline & Ellis y col (1994) & Mutha y col (1997) \\
\hline I (M) & 0.05 & 0 \\
$\mathrm{~s}$ & 0.754218 & 0.6 \\
$\mathrm{~m}$ & 0.238579 & 0.4 \\
$\mathrm{~T}(\mathrm{~K})$ & 333 & 353.15 \\
$\mathrm{~V}(\mathrm{~L})$ & 3.76814 & 1.3 \\
$\mu_{0}$ & 0 & 0 \\
$\mu_{2}$ & 0 & 0 \\
$\mathrm{Q}(\mathrm{K} / \mathrm{min})$ & - & 0 \\
\hline
\end{tabular}

Tabla B.2. Propiedades básicas

\begin{tabular}{|l|c|c|}
\hline & Ellis y col (1994) & Mutha y col (1997) \\
\hline Peso molecular del monómero $\left(\mathrm{PM}_{\mathrm{m}}\right)$ & $100.12(\mathrm{MMA})$ & 100.12 (MMA) \\
Peso molecular del solvente $\left(\mathrm{PM}_{\mathrm{s}}\right)$ & $88($ acetato de etilo) & 92.15 (tolueno) \\
Peso molecular del iniciador $\left(\mathrm{PM}_{\mathrm{l}}\right)$ & 164.21 (AIBN) & 164.21 (AIBN) \\
Densidad del monómero puro $\left(\rho_{\mathrm{m}}^{\circ}, \mathrm{g} / \mathrm{L}\right)$ & 896.689 & 878.5 \\
Densidad del solvente puro $\left(\rho_{\mathrm{s}}^{\circ}, \mathrm{g} / \mathrm{L}\right)$ & 850.966 & 811 \\
Densidad del polímero puro $\left(\rho_{\mathrm{p}}^{\circ}, \mathrm{g} / \mathrm{L}\right)$ & 1175.007 & 1200 \\
\hline
\end{tabular}

Tabla B.3. Constantes de reacción

\begin{tabular}{|c|c|c|}
\hline & Ellis y col (1994) & Mutha y col (1997) \\
\hline $\begin{array}{c}\text { Disociación } \\
A_{D} \\
B_{D} \\
f_{d} \text { (factor de disociación) } \\
\end{array}$ & $\begin{array}{c}38.68508 \\
15430.29 \\
0.58 \\
\end{array}$ & $\begin{array}{c}38.68508 \\
15428.744 \\
0.58 \\
\end{array}$ \\
\hline $\begin{array}{c}\text { Propagación sin gel } \\
A_{p 0} \\
B_{p 0}\end{array}$ & $\begin{array}{l}17.1999 \\
2190.74 \\
\end{array}$ & $\begin{array}{l}18.39228 \\
2609.199 \\
\end{array}$ \\
\hline $\begin{array}{c}\text { Terminación sin gel } \\
A_{40} \\
B_{10} \\
\end{array}$ & $\begin{array}{c}22.49482 \\
352.793 \\
\end{array}$ & $\begin{array}{c}22.49482 \\
352.758 \\
\end{array}$ \\
\hline $\begin{array}{c}\text { Transferencia a monómero } \\
\mathrm{A}_{\mathrm{MM}} \\
\mathrm{B}_{\mathrm{PM}}\end{array}$ & $\begin{array}{c}26.788492 \\
9082.03031\end{array}$ & $\begin{array}{c}9.15694 \\
6733.092\end{array}$ \\
\hline $\begin{array}{c}\text { Transferencia a solvente } \\
\mathbf{A}_{\mathbf{E}} \\
B_{\mathrm{E}}\end{array}$ & $\begin{array}{c}24.05683 \\
7179.45634\end{array}$ & $\begin{array}{c}6.9177 \\
5736.715\end{array}$ \\
\hline
\end{tabular}


Tabla B.4. Efecto gel

\begin{tabular}{|c|c|}
\hline \multicolumn{2}{|c|}{$\begin{array}{c}\text { Parámetros para la función } \mathrm{A}(\mathrm{T}) \\
\text { (Volumen libre) }\end{array}$} \\
\hline $\mathrm{A}_{\mathrm{A}}$ & 0.1678 \\
$\mathrm{~B}_{\mathrm{A}}$ & 0 \\
$\mathrm{C}_{\mathrm{A}}$ & 1.23517 \\
$\mathrm{~T}_{\mathrm{g}}(\mathrm{K})$ & 387.15 \\
\hline Parámetros para la función $\mathrm{B}(\mathrm{T})$ \\
\hline \multicolumn{2}{|c|}{ (Volumen libre) } \\
\hline $\mathrm{A}_{\mathrm{B}}$ & 0.03 \\
$\mathrm{~B}_{\mathrm{B}}$ & 0 \\
\hline \multicolumn{2}{|c|}{ Tiempo de propagación } \\
\hline $\mathrm{A}_{\mathrm{tp}}$ & 35.11094 \\
$\mathrm{~B}_{\mathrm{tp}}$ & 13964 \\
\hline Tiempo de terminación \\
\hline $\mathrm{A}_{\mathfrak{t t}}$ & 47.03 \\
$\mathrm{~B}_{\mathrm{tt}}$ & 48.85 \\
$\mathrm{C}_{\mathrm{t}}$ & 637.19 \\
$\mathrm{D}_{\mathrm{t}}$ & 17956 \\
\hline
\end{tabular}

Valores adicionales para el reactor de Ellis y col (1994)

Cargas de monómero, solvente e iniciador (g)

$\mathrm{w}_{\mathrm{m}}=806.148$

$W_{\mathrm{s}}=2418.444$

$\mathrm{w}_{\mathrm{I}}=30.93965$

Volúmenes de solvente e iniciador (L)

$\mathrm{V}_{\mathrm{s}}=\mathbf{2 . 8 4 2}$

$V_{I}=0.02714$

Valores adicionales para el reactor de Mutha y col (1997)

Concentraciones de entrada de monómero, solvente, momentos 0 y 2 , temperatura de entrada y flujos de entrada y salida

$$
\begin{aligned}
& \mathrm{m}_{\mathrm{e}}=0.4 \\
& \mathrm{~s}_{\mathrm{e}}=0.6 \\
& \mu_{0_{\mathrm{e}}}=0 \\
& \mu_{2 \mathrm{e}}=0 \\
& \mathrm{~T}_{\mathrm{e}}=353.15 \mathrm{~K} \\
& \mathrm{q}_{\mathrm{e}}=0.03333 \mathrm{~L} / \mathrm{min}
\end{aligned}
$$




$$
\begin{aligned}
& q=-\left(\varepsilon_{m} r_{\text {pol }}-\varepsilon_{s} r_{\text {soll }}\right) V+q_{e}+\frac{4}{5}\left(V-V_{B}\right) \\
& V_{B}=1.3 L \text { (volumen de referencia) }
\end{aligned}
$$

Entalpía de propagación (cal/mol)

$$
-\Delta H_{p}=13782
$$

Capacidad calorífica del mon+sol+pol (cal/g-K)

$$
C_{P}=0.4
$$




\section{Apéndice C. Indice de refracción}

En este apéndice se describe una expresión del indice de refracción $\eta$ (y sus derivadas) de una solución de monómero, polímero y solvente, en función de monómero y solvente. Para la descripción de tal expresión se emplea el incremento específico del índice de refracción.

Incremento específico del índice de refracción

El incremento específico del índice de refracción:

$v=\frac{d \eta}{d c}$

es el valor límite cuando $c_{p o l} \rightarrow 0$ de la cantidad $\left(\eta-\eta_{0}\right) / c_{p o l}$, donde $\eta$ y $\eta_{0}$ son los índices de refracción de la solución y el solvente respectivamente y $c_{p o l}(\mathrm{~g} / \mathrm{ml})$ es la concentración de polímero en la solución.

Relación del incremento del índice de refracción y la concentración de polimero

La relación entre $v$ y la concentración de polímero $\left(c_{p o l}\right)$ es:

$\frac{\eta-\eta_{0}}{c_{p o l}}=v+a_{2} c_{p o l}$

Lorimer (1972) desarrolló una expresión para el coeficiente $a_{2}$ en términos de $\eta_{0} \mathrm{y} v$.

$a_{2}=\frac{\left(3 \eta_{0}^{2}-2\right) v^{2}}{2 \eta_{0}\left(\eta_{0}^{2}+2\right)}$

A partir de (C2) la expresión para el índice de refracción es:

$\eta=\eta_{0}+v c_{p o l}+a_{2} c_{p o l}^{2}$

Para una solución de monómero, polímero y solvente, al monómero se le considera como otro solvente y $\eta_{0}$ está dado por:

$\eta_{0}=\eta_{\mathrm{m}} \mathrm{m}_{-}+\eta_{\mathrm{s}} \mathrm{s}_{-}$

donde $\eta_{m}$ y $\eta_{s}$ son los índices de refracción del monómero y solvente, y $m$. y $s$. son las fracciones masa del monómero y solvente en la mezcla monómero + solvente ( $\sin$ polímero).

La expresión (C5) se reescribe en términos de m y s como: 


$$
\eta_{0}(m, s)=\frac{\eta_{m} \rho_{m}^{0} m+\eta_{s} \rho_{s}^{0} s}{\rho_{m}^{0} m+\rho_{s}^{0} s}
$$

La concentración de polímero en la solución se obtiene mediante un balance de materia:

$$
c_{p o l}(m, s)=\frac{\rho_{m}^{0}}{1-\varepsilon_{m}}(1-m-s)
$$

En resumen, la expresión para el índice de refracción y las funcionalidades que contiene son:

$$
\begin{aligned}
& \eta(m, s)=\eta_{0}(m, s)+v c_{p o l}(m, s)+a_{2}(m, s) c_{p o l}^{2}(m, s) \\
& \eta_{0}(m, s)=\frac{\eta_{m} \rho_{m}^{0} m+\eta_{s} \rho_{s}^{0} s}{\rho_{m}^{0} m+\rho_{s}^{0} s} \\
& c_{p o l}(m, s)=\frac{\rho_{m}^{0}}{1-\varepsilon_{m}}(1-m-s) \\
& a_{2}(m, s)=\frac{\left[3 \eta_{0}^{2}(m, s)-2\right] v^{2}}{2 \eta_{0}(m, s)\left[\eta_{0}^{2}(m, s)+2\right]}
\end{aligned}
$$

Derivada parcial de indice de refracción con respecto al monómero

En este punto se probará analíticamente mediante la derivada del índice de refracción con respecto al monómero, que esta medición depende de la concentración de monómero.

Siendo la expresión para el indice de refracción en función de monómero y solvente:

$$
\eta(m, s)=\eta_{0}(m, s)+v c_{p o l}(m, s)+a_{2}(m, s) c_{p o l}^{2}(m, s)
$$

la derivada con respecto al monómero es

$$
\frac{\partial \eta}{\partial m}=\frac{\partial \eta_{0}}{\partial m}+v \frac{\partial c_{p o l}}{\partial m}+c_{p o l}^{2} \frac{\partial a_{2}}{\partial m}+2 a_{2} c_{p o l} \frac{\partial c_{p o l}}{\partial m}
$$

desarrollando cada término de la derivada

$$
\begin{aligned}
& \frac{\partial \eta_{0}}{\partial m}=-\frac{\rho_{m}^{0}\left(\eta_{0}-\eta_{m}\right)}{\rho_{m}^{0} m+\rho_{s}^{0} s} \\
& \frac{\partial c_{p o l}}{\partial m}=-\frac{\rho_{m}^{0}}{1-\varepsilon_{m}}
\end{aligned}
$$


$\frac{\partial \mathrm{a}_{2}}{\partial \mathrm{m}}=\mathrm{f}\left(\eta_{0}, \mathrm{a}_{2}\right) \frac{\partial \eta_{0}}{\partial \mathrm{m}}$

$f\left(\eta_{0}, a_{2}\right)=-\frac{3 a_{2}}{\eta_{0}\left(\eta_{0}^{2}+2\right)}\left(\eta_{0}^{2}-\frac{v^{2}}{a_{2}} \eta_{0}+\frac{2}{3}\right)$

es decir, aunque se verifique que los índices de refracción del monómero y solvente son iguales (es decir, $\eta_{m}=\eta_{s}=\eta_{0}$-a partir de C9a-, haciendo que C11a y C11c se igualen a cero), la derivada de la concentración de polímero con respecto al monómero nunca es igual a cero (C11b), con lo que $\frac{\partial \eta}{\partial \mathrm{m}} \neq 0$

Determinante del jacobiano de densidad e indice de refracción con respecto a $m$ y $s$ El mapa $[\rho, \eta]$ es resoluble para $m$ y $s$ si el siguiente determinante es diferente de cero:

$\operatorname{det}\left[\begin{array}{ll}\frac{\partial \rho}{\partial m} & \frac{\partial \rho}{\partial s} \\ \frac{\partial \eta}{\partial m} & \frac{\partial \eta}{\partial s}\end{array}\right]=\frac{\partial \rho}{\partial m} \frac{\partial \eta}{\partial s}-\frac{\partial \rho}{\partial s} \frac{\partial \eta}{\partial m} \neq 0$

Las derivadas de la densidad con respecto a monómero y solvente son:

$\frac{\partial \rho}{\partial m}=-\frac{\varepsilon_{m} \rho_{m}^{\circ}}{1-\varepsilon_{m}} \quad \frac{\partial \rho}{\partial m}=-\frac{\varepsilon_{s} \rho_{m}^{\circ}}{1-\varepsilon_{m}}$

sustituyendo en (C12) e igualando a cero para determinar las condiciones para las que el conjunto de mediciones no es $m, s$-resoluble, se obtiene:

$\left(-\frac{\varepsilon_{m} \rho_{m}^{\circ}}{1-\varepsilon_{m}}\right) \frac{\partial \eta}{\partial s}-\left(-\frac{\varepsilon_{s} \rho_{m}^{\circ}}{1-\varepsilon_{m}}\right) \frac{\partial \eta}{\partial m}=0$

o bien

$\varepsilon_{\mathrm{m}} \frac{\partial \eta}{\partial \mathrm{s}}-\varepsilon_{\mathrm{s}} \frac{\partial \eta}{\partial \mathrm{m}}=0$

Si (C14) se desarrolla término a término: 


$$
\begin{aligned}
\varepsilon_{\mathrm{m}} \frac{\partial \eta}{\partial \mathrm{s}}-\varepsilon_{\mathrm{s}} \frac{\partial \eta}{\partial \mathrm{m}}= & \varepsilon_{\mathrm{m}}\left[\frac{\partial \eta_{0}}{\partial \mathrm{s}}+v \frac{\partial \mathrm{c}_{\mathrm{pol}}}{\partial \mathrm{s}}+\mathrm{c}_{\mathrm{pol}}^{2} \frac{\partial \mathrm{a}_{2}}{\partial \mathrm{s}}+2 \mathrm{a}_{2} \mathrm{c}_{\mathrm{pol}} \frac{\partial \mathrm{c}_{\mathrm{pol}}}{\partial \mathrm{s}}\right] \\
& -\varepsilon_{\mathrm{s}}\left[\frac{\partial \eta_{0}}{\partial \mathrm{m}}+v \frac{\partial \mathrm{c}_{\mathrm{pol}}}{\partial \mathrm{m}}+\mathrm{c}_{\mathrm{poll}}^{2} \frac{\partial \mathrm{a}_{2}}{\partial \mathrm{m}}+2 \mathrm{a}_{2} \mathrm{c}_{\mathrm{pol}} \frac{\partial \mathrm{c}_{\mathrm{pol}}}{\partial \mathrm{m}}\right] \\
= & \varepsilon_{\mathrm{m}} \frac{\partial \eta_{0}}{\partial \mathrm{s}}-\varepsilon_{\mathrm{s}} \frac{\partial \eta_{0}}{\partial \mathrm{m}}+v\left(\varepsilon_{\mathrm{m}} \frac{\partial \mathrm{c}_{\mathrm{pol}}}{\partial \mathrm{s}}-\varepsilon_{\mathrm{s}} \frac{\partial c_{\mathrm{pol}}}{\partial \mathrm{m}}\right) \\
& +\mathrm{c}_{\mathrm{pol}}^{2}\left(\varepsilon_{\mathrm{m}} \frac{\partial \mathrm{a}_{2}}{\partial \mathrm{s}}-\varepsilon_{\mathrm{s}} \frac{\partial \mathrm{a}_{2}}{\partial \mathrm{m}}\right)+2 \mathrm{a}_{2} \mathrm{c}\left(\varepsilon_{\mathrm{m}} \frac{\partial c_{\mathrm{pol}}}{\partial \mathrm{s}}-\varepsilon_{\mathrm{s}} \frac{\partial \mathrm{c}_{\mathrm{pol}}}{\partial \mathrm{m}}\right)
\end{aligned}
$$

El determinante es igual a cero si todos los términos del lado derecho de (C15) son iguales a cero. Designando como $t_{1}, \ldots, t_{4}$ a los términos del lado derecho de (C15), se realiza el análisis para los que cada término es igual a cero:

$$
t_{1}=\varepsilon_{m} \frac{\partial \eta_{0}}{\partial s}-\varepsilon_{s} \frac{\partial \eta_{0}}{\partial m}=\frac{1}{\rho_{m}^{0} m+\rho_{s}^{0} s}\left[-\varepsilon_{m} \rho_{s}^{0}\left(\eta_{0}-\eta_{s}\right)+\varepsilon_{s} \rho_{m}^{0}\left(\eta_{0}-\eta_{m}\right)\right]
$$

$t_{1}=0$ si se cumple al menos una de las siguientes condiciones:

1) $\rho_{\mathrm{m}}^{0}=\rho_{\mathrm{s}}^{0}=\rho_{\mathrm{p}}^{0}$. Las densidades del monómero y solvente son iguales a la del polímero, por lo que los factores de contracción $\left(\varepsilon_{\mathrm{m}}, \varepsilon_{\mathrm{s}}\right)$ son iguales a cero.

2) $\eta_{m}=\eta_{s}=\eta_{0}$. Los índices de refracción del monómero y solvente son iguales al de la mezcla monómero + solvente.

$t_{2}=v\left(\varepsilon_{m} \frac{\partial c}{\partial s}-\varepsilon_{s} \frac{\partial c}{\partial m}\right)=v\left(-\frac{\varepsilon_{m} \rho_{m}^{\circ}}{1-\varepsilon_{m}}+\frac{\varepsilon_{s} \rho_{m}^{\circ}}{1-\varepsilon_{m}}\right)=-\frac{v \rho_{m}^{0}}{1-\varepsilon_{m}}\left(\varepsilon_{m}-\varepsilon_{s}\right)$

$t_{2}=0$ si se cumple la siguiente condición:

$\rho_{\mathrm{m}}^{\circ}=\rho_{\mathrm{s}}^{\circ}$. Las densidades del monómero y solvente son iguales (si además son iguales a la del polímero, los factores de contracción $-\varepsilon_{\mathrm{m}}, \varepsilon_{\mathrm{s}}$ - son iguales a cero).

$t_{3}=\varepsilon_{m} \frac{\partial a_{2}}{\partial s}-\varepsilon_{s} \frac{\partial a_{2}}{\partial m}=f\left(\eta_{0}, a_{2}\right)\left(\varepsilon_{m} \frac{\partial \eta_{0}}{\partial s}-\varepsilon_{s} \frac{\partial \eta_{0}}{\partial m}\right)=f\left(\eta_{0}, a_{2}\right) t_{1}$

$t_{3}=0$ si se cumple al menos una de las mismas condiciones que para $t_{1}$. 
dodudice $C$

$$
t_{4}=2 a_{2} c_{p o l}\left(\varepsilon_{m} \frac{\partial c}{\partial s}-\varepsilon_{s} \frac{\partial c}{\partial m}\right)=\frac{2 a_{2} c_{p o l}}{v} t_{2}
$$

$t_{4}=0$ si se cumple la misma condición que para $t_{2}$.

En conclusión $[\rho, \eta]$ no es $m, s$-resoluble (determinante igual a cero) si se cumple alguna de las siguientes condiciones:

1) $\rho_{\mathrm{m}}^{\circ}=\rho_{\mathrm{s}}^{0}=\rho_{\mathrm{p}}^{\circ}$ (Las densidades del monómero y solvente son iguales a la del polímero)

2) $\eta_{\mathrm{m}}=\eta_{\mathrm{s}}\left(=\eta_{\mathrm{o}}\right)$ y $\rho_{\mathrm{m}}^{\circ}=\rho_{\mathrm{s}}^{\circ}$ (Los índices de refracción del monómero y solvente son iguales, y lo mismo para las densidades).

79 


\section{Apéndice D. Deducción de las variables de interés para un reactor de} homopolimerización en solución

\section{Densidad}

Para la deducción de la densidad se realiza la suposición de que los volúmenes específicos de monómero, solvente y polímero son aditivos:

$\mathrm{v}=m \mathrm{v}_{\mathrm{m}}^{\mathrm{o}}+\mathrm{pv}_{\mathrm{p}}^{\mathrm{o}}+\mathbf{s v _ { \mathrm { s } } ^ { 0 }}$

donde:

$\mathrm{m}$ : fracción masa de monómero en la solución

$\mathrm{p}$ : fracción masa de polímero en la solución

$s$ : fracción masa de solvente en la solución

las cuales cumplen la relación

$\mathrm{m}+\mathrm{p}+\mathrm{s}=1$

Designando como

$\rho_{\mathrm{m}}^{+}$: cantidad de masa de monómero en una unidad de volumen de la solución

$\rho_{\mathrm{p}}^{+}$: cantidad de masa de polímero en una unidad de volumen de la solución

$\rho_{\mathrm{s}}^{+}$: cantidad de masa de solvente en una unidad de volumen de la solución

la densidad es entonces

$\rho=\rho_{\mathrm{m}}^{+}+\rho_{\mathrm{p}}^{+}+\rho_{\mathrm{s}}^{+}$

o en términos de las fracciones masa y las densidades de los compuestos puros

$\rho=m \rho_{m}^{0}+p \rho_{p}^{\circ}+s \rho_{s}^{0}$

sustituyendo (D2) en (D4)

$\rho=m \rho_{m}^{\circ}+(1-m-s) \rho_{p}^{\circ}+s \rho_{s}^{\circ}$

$\rho=-\left(\rho_{\mathrm{p}}^{0}-\rho_{\mathrm{m}}^{0}\right) m-\left(\rho_{\mathrm{p}}^{0}-\rho_{\mathrm{s}}^{0}\right) s+\rho_{\mathrm{p}}^{0}$

designando como factores de contracción para el monómero y solvente: 


$$
\varepsilon_{\mathrm{m}}=1-\frac{\rho_{\mathrm{m}}^{0}}{\rho_{\mathrm{p}}^{0}} \quad \varepsilon_{\mathrm{s}}=1-\frac{\rho_{\mathrm{s}}^{0}}{\rho_{\mathrm{p}}^{0}}
$$

la ecuación (D5) se expresa

$\rho=-\varepsilon_{\mathrm{m}} \rho_{\mathrm{p}}^{0} \mathrm{~m}-\varepsilon_{\mathrm{s}} \rho_{\mathrm{p}}^{0} \mathrm{~s}+\rho_{\mathrm{p}}^{0}$

sustituyendo $\rho_{\mathrm{p}}^{\circ}$ a partir de (D6a) se obtiene la expresión para la densidad

$\rho=\frac{\rho_{\mathrm{m}}^{0}}{1-\varepsilon_{\mathrm{m}}}\left(1-\varepsilon_{\mathrm{m}} \mathrm{m}-\varepsilon_{\mathrm{s}} \mathrm{s}\right)$

Densidad (Ellis y col., 1994)

Para este caso, las fracciones masa cumplen la relación

$$
m+p+\frac{V_{s}}{V}+\frac{V_{I}}{V}=1
$$

en donde se sustituyó $s=V_{s}($ Volumen inicial de solvente $) / V$ y se define una fracción masa para el iniciador como $V_{V} / V$.

Designando como

$\rho_{1}^{+}$: cantidad de masa de iniciador en una unidad de volumen de la solución

la densidad es entonces

$\rho=\rho_{m}^{+}+\rho_{p}^{+}+\rho_{s}^{+}+\rho_{I}^{+}$

o en términos de las fracciones masa y las densidades de los compuestos puros

$$
\rho=m \rho_{m}^{0}+p \rho_{p}^{0}+\frac{V_{s}}{V} \rho_{s}^{0}+\frac{V_{1}}{V} \rho_{1}^{0}
$$

sustituyendo (D8)

$$
\begin{aligned}
& \rho=m \rho_{m}^{0}+\left(1-m-\frac{V_{s}}{V}-\frac{V_{I}}{V}\right) \rho_{p}^{0}+\frac{V_{s}}{V} \rho_{s}^{0}+\frac{V_{l}}{V} \rho_{I}^{0} \\
& \rho=-\left(\rho_{p}^{0}-\rho_{m}^{0}\right) m-\left(\rho_{p}^{0}-\rho_{s}^{0}\right) \frac{V_{s}}{V}-\left(\rho_{p}^{0}-\rho_{i}^{0}\right) \frac{V_{1}}{V}+\rho_{p}^{0}
\end{aligned}
$$

designando como factor de contracción para el iniciador

$\varepsilon_{1}=1-\frac{\rho_{1}^{0}}{\rho_{p}^{0}}$

la ecuación (D11) se expresa 
$\rho=-\varepsilon_{m} \rho_{p}^{0} m-\varepsilon_{s} \rho_{p}^{0} \frac{V_{s}}{V}-\varepsilon_{1} \rho_{p}^{0} \frac{V_{1}}{V}+\rho_{p}^{0}$

sustituyendo $\rho_{\mathrm{p}}^{\circ}$ a partir de (D6a) se obtiene la expresión para la densidad

$\rho=\frac{\rho_{m}^{0}}{1-\varepsilon_{m}}\left(1-\varepsilon_{m} m-\varepsilon_{s} \frac{V_{s}}{V}-\varepsilon_{I} \frac{V_{I}}{V}\right)$

Densidad (Mutha y col, 1997)

La expresión para la conversión (en función de la densidad) reportada por Mutha y col. es la siguiente:

$$
c=\frac{\frac{\rho_{e}}{\rho}-1}{1-\frac{\rho_{p}^{0}}{\rho_{m}^{0}}}
$$

a partir del factor de contracción para el monómero (D6a) se obtiene:

$$
\frac{\rho_{p}^{0}}{\rho_{m}^{0}}=\frac{1}{1-\varepsilon_{m}}
$$

y sustituyendo (D6a) y (D15) en (D14) se obtiene:

$$
1-\frac{\varepsilon_{m}}{1-\varepsilon_{m}} c=\frac{\rho_{e}}{\rho}
$$

Siendo la expresión para la conversión en función de $m$ y $s$ la siguiente

$$
c=\frac{1-m-s}{1-s}
$$

la expresión para la densidad es:

$$
\rho=\frac{(1-s)\left(1-\varepsilon_{m}\right)}{(1-s)-\varepsilon_{m}(2-2 s-m)} \rho_{e}
$$

\section{Conversión}

La conversión se define como

$$
c=\frac{\text { masa de polimero }}{\text { masa de monomero }+ \text { masa de polimero }}
$$




$$
c=\frac{p \rho_{p}^{0}}{m \rho_{m}^{0}+p \rho_{p}^{0}}=\frac{1-m-s}{m \frac{\rho_{m}^{0}}{\rho_{p}^{\circ}}+1-m-s}=\frac{1-m-s}{1-\varepsilon_{m} m-s}
$$

Conversión (Ellis y col., 1994)

La expresión para la conversión reportada por Ellis y col. (1994) es la siguiente:

$$
c=\frac{\frac{w_{T}}{\rho}-\frac{w_{m}}{\rho_{m}^{0}}-\frac{w_{s}}{\rho_{s}^{0}}-\frac{w_{l}}{\rho_{l}^{0}}}{\frac{w_{m}}{\rho_{p}^{0}}-\frac{w_{m}}{\rho_{m}^{0}}}
$$

Conversión (Mutha y col., 1997)

La expresión original reportada para la conversión predicha es:

$c=\frac{[\mathrm{P}]}{[\mathrm{M}]+[\mathrm{P}]}$

o con variables adimensionales:

$$
c=\frac{p}{m+p}=\frac{1-m-s}{1-s}
$$

En función de la densidad, a partir de (D16) la conversión es:

$$
c=\frac{1-\varepsilon_{\mathrm{m}}}{\varepsilon_{\mathrm{m}}}\left(1-\frac{\rho_{\mathrm{e}}}{\rho}\right)
$$

Fracción masa de sólidos

La fracción masa de sólidos se define como

$$
\begin{aligned}
& \phi=\frac{\text { masa de polimero }}{\text { masa total }} \\
& \phi=\frac{p \rho_{\mathrm{p}}^{0}}{\frac{\rho_{\mathrm{m}}^{\circ}}{1-\varepsilon_{\mathrm{m}}}\left(1-\varepsilon_{\mathrm{m}} \mathrm{m}-\varepsilon_{\mathrm{s}} s\right)}=\frac{1-\mathrm{m}-\mathrm{s}}{1-\varepsilon_{\mathrm{m}} \mathrm{m}-\varepsilon_{\mathrm{s}} \mathrm{s}}
\end{aligned}
$$


Fracción masa de sólidos (Ellis y col., 1994)

A partir de (D22a), (D8) y (D13), la fracción de sólidos es

$$
\phi=\frac{p \rho_{p}^{0}}{\frac{\rho_{m}^{0}}{1-\varepsilon_{m}}\left(1-\varepsilon_{m} m-\varepsilon_{s} \frac{V_{s}}{V}-\varepsilon_{1} \frac{V_{I}}{V}\right)}=\frac{1-m-\frac{V_{s}}{V}-\frac{V_{I}}{V}}{1-\varepsilon_{m} m-\varepsilon_{s} \frac{V_{s}}{V}-\varepsilon_{I} \frac{V_{I}}{V}}
$$

Fracción masa de sólidos (Mutha y col., 1997)

De acuerdo con (D22a), la fracción masa de sólidos es

$$
\phi=\frac{[\mathrm{P}]}{[\mathrm{M}]+[\mathrm{P}]+[\mathrm{S}]}
$$

y procediendo de forma similar que con la conversión, la fracción de sólidos queda expresada mediante variables adimensionales como sigue

$$
\phi=\frac{p}{m+p+s}=1-m-s
$$

\section{Peso molecular en número}

De (D2) se tiene

$$
\mathrm{p}=1-\mathrm{m}-\mathrm{s}
$$

La concentración másica de polímero en la solución es:

$$
\rho_{\mathrm{p}}^{+}=\mathrm{p} \rho_{\mathrm{p}}^{\circ}=(1-\mathrm{m}-\mathrm{s}) \rho_{\mathrm{p}}^{\circ}
$$

sustituyendo $\rho_{\mathrm{p}}^{\circ}$ a partir de (D6a) y dividiendo entre $\left(\mathrm{PM}_{\mathrm{m}}\right)$

$$
\mu_{1}=\frac{M^{0}}{1-\varepsilon_{\mathrm{m}}}(1-\mathrm{m}-\mathrm{s})
$$

El peso molecular en número se define como

$$
M_{n}=P_{m} \frac{\mu_{i}}{\mu_{0}}
$$

sustituyendo (D26) en (D27a) se obtiene

$$
M_{\mathrm{n}}=\frac{\rho_{m}^{0}(1-m-s)}{\left(1-\varepsilon_{m}\right) \mu_{0}}
$$




\section{Peso molecular en peso}

El peso molecular en peso se define como

$$
M_{w}=P M_{m} \frac{\mu_{2}}{\mu_{1}}
$$

sustituyendo (D26) en (D28a) se obtiene

$$
M_{w}=\frac{P M_{m}^{2} \mu_{2}\left(1-\varepsilon_{m}\right)}{\rho_{m}^{0}(1-m-s)}
$$




\section{Apéndice E. Construcción y sintonización de los estimadores no lineales}

La construcción y sintonización de los estimadores se realizará conforme al procedimiento descrito en el capítulo III.

\section{Construcción}

Mediciones e indices de observabilidad

El conjunto de mediciones es

$y=[\rho(m, s, v), T, v]^{T}$

para el que las trayectorias del reactor son RE - estimables con los siguientes conjuntos de índices de observabilidad

$\mathrm{C}_{1}=\{1,1,1\}, \mathrm{C}_{2}=\{2,1,1\}$

Mapas de estimabilidad

Los mapas de estimabilidad correspondientes a $\mathrm{C}_{1}$ y $\mathrm{C}_{2}$ son:

$$
\begin{aligned}
& \Phi=\left[\begin{array}{llllllll}
\rho(m, s, V) & T & V & I & s & \mu_{0} & \mu_{2}
\end{array}\right]^{T} \\
& \Phi=\left[\begin{array}{llllllll}
\rho & \frac{\partial \rho}{\partial m} f_{m}+\frac{\partial \rho}{\partial s} f_{s}+\frac{\partial \rho}{\partial V} f_{V} & T & V & s & m_{0} & m_{2}
\end{array}\right]^{T}
\end{aligned}
$$

Jacobianos e inversas

Los jacobianos de los mapas de estimabilidad y sus inversas se enumeran a continuación. La nomenclatura de las derivadas parciales utilizadas es la siguiente:

$$
\rho_{i}=\frac{\partial \rho}{\partial i} \quad \dot{\rho}_{i}=\frac{\partial \dot{\rho}}{\partial i} \quad ; i: I, m, s, T, V
$$


para $\mathrm{C}_{1}$ :

$$
\Phi_{\mathrm{x}}=\left[\begin{array}{ccccccc}
0 & \rho_{\mathrm{s}} & \rho_{\mathrm{m}} & 0 & \rho_{\mathrm{v}} & 0 & 0 \\
0 & 0 & 0 & 1 & 0 & 0 & 0 \\
0 & 0 & 0 & 0 & 1 & 0 & 0 \\
1 & 0 & 0 & 0 & 0 & 0 & 0 \\
0 & 1 & 0 & 0 & 0 & 0 & 0 \\
0 & 0 & 0 & 0 & 0 & 1 & 0 \\
0 & 0 & 0 & 0 & 0 & 0 & 1
\end{array}\right] \quad \Phi_{\mathrm{x}}^{-1}=\left[\begin{array}{ccccccc}
0 & 0 & 0 & 1 & 0 & 0 & 0 \\
0 & 0 & 0 & 0 & 1 & 0 & 0 \\
\frac{1}{\rho_{\mathrm{m}}} & 0 & -\frac{\rho_{\mathrm{v}}}{\rho_{\mathrm{m}}} & 0 & -\frac{\rho_{\mathrm{s}}}{\rho_{\mathrm{m}}} & 0 & 0 \\
0 & 1 & 0 & 0 & 0 & 0 & 0 \\
0 & 0 & 1 & 0 & 0 & 0 & 0 \\
0 & 0 & 0 & 0 & 0 & 1 & 0 \\
0 & 0 & 0 & 0 & 0 & 0 & 1
\end{array}\right]
$$

para $\mathrm{C}_{2}$ :

$$
\begin{aligned}
& \Phi_{\mathrm{x}}=\left[\begin{array}{ccccccc}
0 & \rho_{\mathrm{s}} & \rho_{\mathrm{m}} & 0 & \rho_{\mathrm{v}} & 0 & 0 \\
\dot{\rho}_{1} & \dot{\rho}_{\mathrm{s}} & \dot{\rho}_{\mathrm{m}} & \dot{\rho}_{\mathrm{T}} & \dot{\rho}_{\mathrm{V}} & 0 & 0 \\
0 & 0 & 0 & 1 & 0 & 0 & 0 \\
0 & 0 & 0 & 0 & 1 & 0 & 0 \\
0 & 1 & 0 & 0 & 0 & 0 & 0 \\
0 & 0 & 0 & 0 & 0 & 1 & 0 \\
0 & 0 & 0 & 0 & 0 & 0 & 1
\end{array}\right]
\end{aligned}
$$

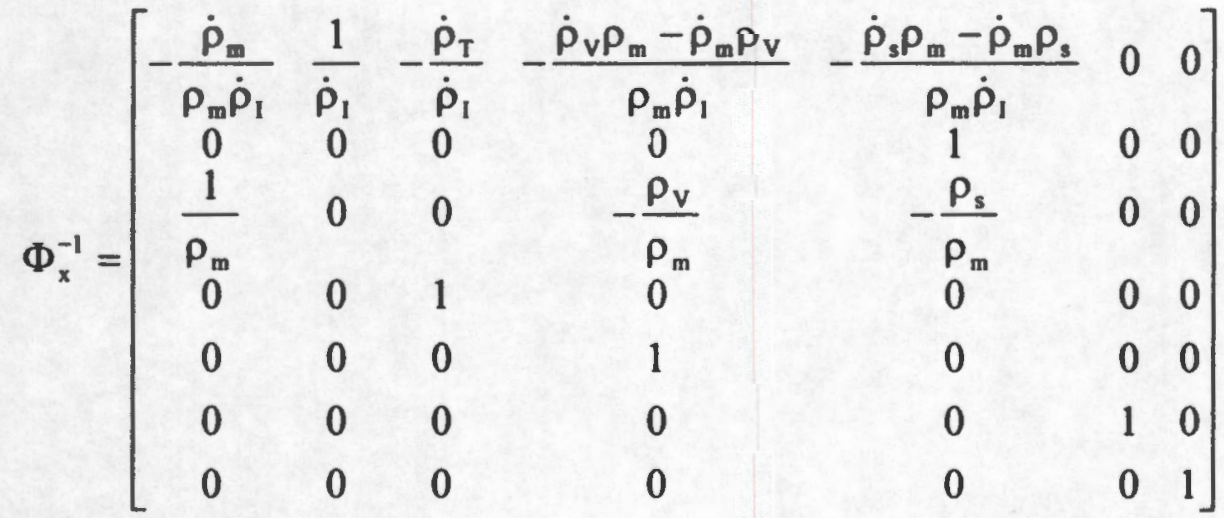

\section{Matriz lineal proporcional}

Las matrices lineales proporcionales correspondientes, de acuerdo con (3.14b) son: 
$K_{0}\left(s_{o}\right)=\left[\begin{array}{ccc}s_{0} k_{p}^{0} & 0 & 0 \\ 0 & s_{0} k_{T}^{0} & 0 \\ 0 & 0 & s_{0} k_{v}^{\circ}\end{array}\right]$

$K_{o}\left(s_{0}\right)=\left[\begin{array}{ccc}s_{0} k_{1 y_{s}}^{o} & 0 & 0 \\ s_{o}^{2} k_{2 \rho}^{0} & 0 & 0 \\ 0 & s_{0} k_{T}^{o} & 0 \\ 0 & 0 & s_{0} k_{v}^{o}\end{array}\right]$

Matriz no lineal $G_{p}$

La matriz no lineal $G_{p}$ se obtiene de acuerdo con (3.16a), en donde $\theta_{1}$ consiste de las primeras $\kappa$ columnas de $\Phi_{x}^{-1}$. Para cada uno de los conjuntos, la matriz $\mathrm{G}_{\mathrm{p}}$ es:

para $C_{1}$ :

$G_{p}=\left[\begin{array}{ccc}0 & 0 & 0 \\ s_{0} k_{p}^{0}\left(\frac{1}{\rho_{m}}\right) & 0 & s_{0} k_{v}^{0}\left(-\frac{\rho_{v}}{\rho_{m}}\right) \\ 0 & s_{0} k_{T}^{0} & 0 \\ 0 & 0 & s_{0} k_{v}^{0} \\ 0 & 0 & 0 \\ 0 & 0 & 0\end{array}\right]$

para $\mathrm{C}_{2}$ :

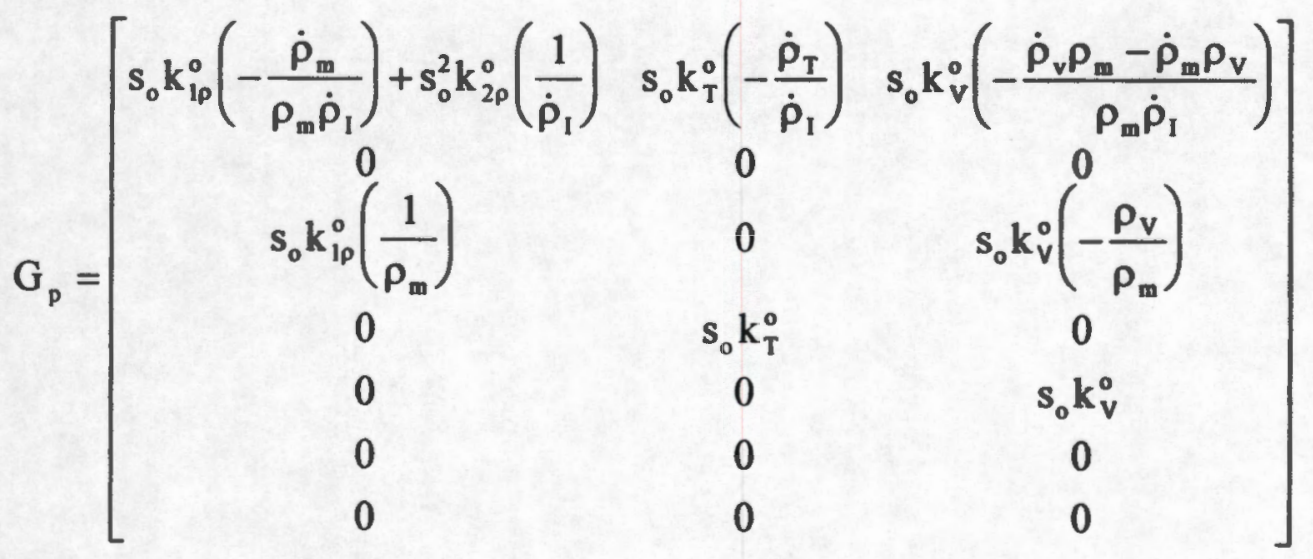

(E4b)

Matriz lineal integral

Las matrices lineales integrales correspondientes, de acuerdo con (3.14c) son: 
$K_{I}\left(s_{o}\right)=\left[\begin{array}{ccc}s_{o}^{2} k_{p}^{1} & 0 & 0 \\ 0 & s_{0}^{2} k_{T}^{1} & 0 \\ 0 & 0 & s_{o}^{2} k_{V}^{I}\end{array}\right] \quad K_{I}\left(s_{o}\right)=\left[\begin{array}{ccc}s_{0}^{3} k_{p}^{I} & 0 & 0 \\ 0 & s_{0}^{2} k_{T}^{1} & 0 \\ 0 & 0 & s_{o}^{2} k_{V}^{1}\end{array}\right]$

\section{Matriz no lineal $G_{I}$}

La matriz no lineal $\mathrm{G}_{1}$ se obtiene de acuerdo con (3.16b), en donde $\theta_{1}$ consiste de las primeras $\kappa$ columnas de $\Phi_{\mathrm{x}}^{-1}$. Para cada uno de los conjuntos, la matriz $\mathrm{G}_{\mathrm{l}}$ es:

para $C_{1}$ :

$$
G_{1}=\left[\begin{array}{ccc}
0 & 0 & 0 \\
0 & 0 & 0 \\
s_{0}^{2} k_{p}^{1}\left(\frac{1}{\rho_{m}}\right) & 0 & s_{0}^{2} k_{v}^{I}\left(-\frac{\rho_{v}}{\rho_{m}}\right) \\
0 & s_{0}^{2} k_{T}^{I} & 0 \\
0 & 0 & s_{0}^{2} k_{v}^{I} \\
0 & 0 & 0 \\
0 & 0 & 0
\end{array}\right]
$$

para $\mathrm{C}_{2}$ :

$$
G_{p}=\left[\begin{array}{ccc}
s_{0}^{3} k_{p}^{1}\left(\frac{1}{\dot{\rho}_{I}}\right) & s_{0}^{2} k_{T}^{1}\left(-\frac{\dot{\rho}_{T}}{\dot{\rho}_{1}}\right) & s_{0}^{2} k_{v}^{1}\left(-\frac{\dot{\rho}_{v} \rho_{m}-\dot{\rho}_{m} \rho_{V}}{\rho_{m} \dot{\rho}_{I}}\right) \\
0 & 0 & 0 \\
0 & 0 & s_{0}^{2} k_{v}^{I}\left(-\frac{\rho_{\mathrm{v}}}{\rho_{m}}\right) \\
0 & s_{0}^{2} k_{T}^{1} & 0 \\
0 & 0 & s_{0}^{2} k_{V}^{1} \\
0 & 0 & 0 \\
0 & 0 & 0
\end{array}\right]
$$

El estimador no lineal es de la forma:

$\dot{\chi}=f\left[x, \Delta_{u} \chi_{u}, \rho\right]+G_{p}\left(x, x_{u}, \rho\right)[y-h(x, \rho)]+G_{1}\left(x, x_{u}, \rho\right) \int_{0}[y-h(x, \rho)] d \tau$ 


\section{Sintonización}

Dinámicas de $2^{\circ}$. orden

Las ganancias $\mathbf{k}_{i}^{\circ}$ y $\mathbf{k}_{i}^{1}(i=\rho, T, V)$ son los coeficientes de las dinámicas LNPA de segundo orden de los errores de estimación de las salidas:

$\ddot{v}_{i}+k_{i}^{\circ} \dot{v}_{i}+k_{i}^{1} v_{i}=0$

En términos del factor de frecuencia $\omega$ y del factor de amortiguamiento $\zeta$ (Stephanopoulos, 1984) las dinámicas pueden reescribirse:

$\ddot{v}_{i}+2 \zeta_{i} \omega_{i} \dot{v}_{i}+\omega_{i}^{2} v_{i}=0$

Dado que el factor de frecuencia es el inverso del tiempo crítico $\left(\omega=1 / \tau_{c}\right)$ y que para dinámicas de segundo orden el tiempo de asentamiento es

$\tau_{\mathrm{as}}=4 \tau_{\mathrm{c}} / \zeta$

las expresiones para las constantes del estimador son:

$$
k_{i}^{\circ}=\frac{8}{\left(\tau_{\text {ss }}\right)_{i}} \quad k_{i}^{\prime}=\frac{16}{\left(\zeta \tau_{\text {as }}\right)_{i}^{2}}
$$

\section{Dinámica de $3^{\text {er }}$.orden}

Las ganancias $\mathrm{k}_{\mathrm{i}}^{\circ}$, $\mathrm{k}_{2 \mathrm{i}}^{\circ}$ y $\mathbf{k}_{\mathrm{i}}^{\mathrm{l}}(\mathrm{i}=\rho)$ son los coeficientes de las dinámicas LNPA de tercer orden de los errores de estimación de las salida:

$\ddot{v}_{i}+k_{l i}^{0} \ddot{v}_{i}+k_{2 i}^{o} \dot{v}_{i}+k_{i}^{1} v_{i}=0$

en la que las constantes para el estimador están definidas en términos del tiempo de asentamiento y el factor de amortiguamiento:

$$
k_{1 i}^{o}=\frac{12}{\left(\tau_{\mathrm{a}}\right)_{i}} \quad k_{2 i}^{o}=\frac{16}{\left(\tau_{\mathrm{a}}\right)_{i}^{2}}\left(2+\frac{1}{\zeta_{i}^{2}}\right) \quad k_{i}^{1}=\frac{64}{\zeta_{i}^{2}\left(\tau_{\mathrm{a}}\right)_{i}^{3}}
$$

